\title{
Two-photon exchange in elastic electron-proton scattering
}

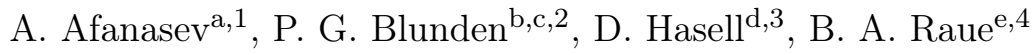 \\ ${ }^{a}$ George Washington University, Washington, D.C., U.S.A. \\ ${ }^{b}$ University of Manitoba, Winnipeg, MB, Canada \\ ${ }^{c}$ Jefferson Lab, Newport News, VA, U.S.A. \\ ${ }^{d}$ Massachusetts Institute of Technology, Cambridge, MA, U.S.A. \\ ${ }^{e}$ Florida International University, Miami, FL, U.S.A.
}

\begin{abstract}
We review recent theoretical and experimental progress on the role of two-photon exchange (TPE) in electron-proton scattering at low to moderate momentum transfers. We make a detailed comparison and analysis of the results of competing experiments on the ratio of $e^{+} p$ to $e^{-} p$ elastic scattering cross sections, and of the theoretical calculations describing them. A summary of the current experimental situation is provided, along with an outlook for future experiments.
\end{abstract}

Keywords: Two-photon exchange, Radiative corrections, Form factors

Preprint: JLAB-THY-17-2404

\footnotetext{
1afanas@gwu.edu

${ }^{2}$ blunden@physics.umanitoba.ca

${ }^{3}$ hasell@mit.edu

${ }^{4}$ baraue@fiu.edu
} 


\section{Contents}

1 Introduction $\quad 3$

2 Theoretical overview $\quad 4$

2.1 Kinematics and definitions . . . . . . . . . . . . . . . . . . . . 4

2.2 Experimental measurements of proton form factors . . . . . . . . . . . . . . . 5

2.3 Two-photon exchange . . . . . . . . . . . . . . . . . . 7

2.3 .1 General formulation . . . . . . . . . . . . . . . . . . . . . 7

2.3.2 Model-independent TPE corrections . . . . . . . . . . . . . . . . 8

2.3 .3 One-loop methods . . . . . . . . . . . . . . . . . . . . . 8

2.3 .4 Dispersive methods . . . . . . . . . . . . . . . . . . . . . 12

2.3.5 QCD-based approaches at high $Q^{2} \ldots \ldots \ldots \ldots \ldots$

2.4 Two-photon exchange for spin polarization effects . . . . . . . . . . . . . . 15

3 Experimental observation of TPE $\quad 19$

3.1 Nonlinearity of Rosenbluth data . . . . . . . . . . . . . . . . . . . . . . . 19

3.2 Direct measurements of TPE corrections to $\sigma_{R} \ldots \ldots \ldots \ldots \ldots$

3.3 Early measurements of TPE . . . . . . . . . . . . . . . . . . . . . . 21

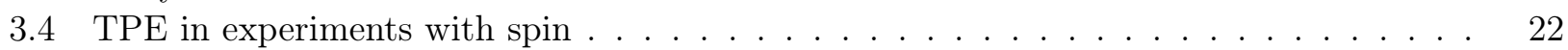

3.5 The VEPP-3 experiment . . . . . . . . . . . . . . . . . . . . . . . . 25

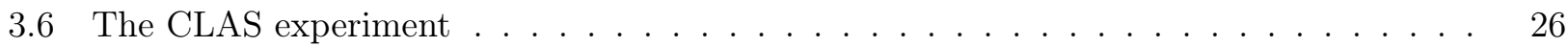

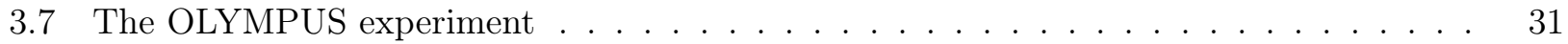

3.7 .1 Luminosity . . . . . . . . . . . . . . . . . . . . . . 32

3.7 .2 Radiative corrections . . . . . . . . . . . . . . . . . . . 33

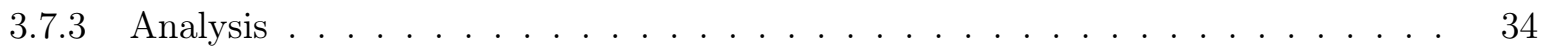

3.8 Comparison of recent experiments and models . . . . . . . . . . . . . 36

4 Conclusions and outlook $\quad 38$ 


\section{Introduction}

Electron scattering has been a primary experimental tool in the study of hadron physics for many decades. The electromagnetic interaction is well understood, and the pointlike nature of electrons makes it an ideal tool to probe the internal structure of hadrons. Furthermore, the relatively small value of the electromagnetic coupling, $\alpha \sim 1 / 137$, makes the electromagnetic interaction amenable to a perturbative treatment in the context of quantum field theory.

Much of our information on the structure of the proton comes from unpolarized measurements of the inclusive electron-proton scattering cross section. These measurements determine the proton electric $\left(G_{E}^{p}\right)$ and magnetic $\left(G_{M}^{p}\right)$ form factors, which are fundamental observables characterizing the internal structure of the proton. Specifically, the quantities $\left(G_{E}^{p}\right)^{2}$ and $\left(G_{M}^{p}\right)^{2}$ can be extracted from the angular dependence of the unpolarized electron scattering cross section.

More recently, polarized beams, polarized targets, and measurements of the recoil polarization of the target proton have been used to provide additional information on the spin structure of the proton, and to improve our knowledge of proton form factors. Polarization measurements have proven to be a crucial ingredient in studies of proton form factors over the past two decades. These experiments access the ratio $G_{E}^{p} / G_{M}^{p}$ directly from the ratio of transverse to longitudinal nuclear polarization measurements.

In what has become known colloquially as "the proton form factor puzzle", a comparison of the form factor ratio extracted from both types of experiments revealed a significant discrepancy in kinematic regions where both techniques provide precise measurements. Because these, and essentially all other electron scattering measurements, are analyzed in the framework of the one-photon exchange (OPE) or Born approximation, this discrepancy led to a reexamination of the possible role played by radiative corrections to the electron scattering cross sections. For electron scattering, radiative corrections must be applied to measured cross sections in order to extract an equivalent OPE form. Although these radiative corrections are large, they are generally model-independent and well understood. In particular, the standard radiative corrections are independent of hadronic structure.

Attempts to reconcile the unpolarized and polarized measurements have mostly focussed on improved treatments of these radiative corrections. Of particular interest, and the subject of this review, are considerations of two-photon exchange (TPE) effects beyond the minimal model-independent terms incorporated into the standard radiative corrections. The challenge in calculating these TPE contributions is that they are not independent of hadronic structure. The challenge in measuring them directly is that they are most prominent at high momentum transfer and backward scattering angles, where the cross section is suppressed. Early measurements and calculations suggested that TPE effects are a few percent correction to cross sections, consistent with the expectation that they are of order $\mathcal{O}(\alpha)$ compared to the OPE approximation. However, there is now convincing evidence that these corrections can nevertheless be extremely important in specific circumstances.

Over the past 15 years there has been a significant investment, on both the theoretical and experimental fronts, to studying TPE in electromagnetic processes. Many of these efforts have been the subject of previous reviews, such as the 2007 review by Carlson and Vanderhaeghen [1], and 2011 review by Arrington, Blunden and Melnitchouk [2]. Since the 2011 review [2] there has been significant progress in theoretical calculations, which we highlight here. In addition, results have recently been reported from the VEPP-3, CLAS, and OLYMPUS experiments, which were designed to directly measure TPE effects from the ratio of $e^{+} p$ to $e^{-} p$ elastic scattering cross sections.

Interest in TPE effects has been furthered by the so-called "proton radius problem" [3]. Briefly, the proton radius extracted from electron scattering and atomic hydrogen spectroscopy measurements disagrees by several standard deviations from the proton radius extracted by spectroscopy on muonic atoms. Two-photon exchange is one contributor to the energy shift in atomic systems. This is described in a recent review by Carlson [4], and we don't address it further in this review.

The outline of this review is as follows. Section 2 provides a theoretical overview. This includes the 
relevant electron scattering formalism in sections 2.1 and 2.2. Section 2.3 describes the formalism and calculations of TPE corrections in unpolarized electron scattering, summarizing the older work but focussing on recent improvements in the past five years. Two-photon exchange for spin-polarization effects are described in section 2.4.

Section 3 focusses on experimental measurements. In particular, the recent VEPP-3, CLAS, and OLYMPUS experiments, which look for direct evidence of TPE effects by measuring the ratio of $e^{+} p$ to $e^{-} p$ elastic cross sections, are each described in some detail. A comparison and analysis of the results of these experiments is made in section 3.8. Conclusions and the outlook for both theory and experiment are given in section 4 .

\section{Theoretical overview}

\subsection{Kinematics and definitions}

In this section we define the general kinematics of elastic electron-nucleon scattering, and present amplitudes and cross sections in the OPE or Born approximation.

For the elastic scattering process $e N \rightarrow e N$ (see Fig. 2.1), the four-momenta of the initial and final electrons (mass $m_{e}$ ) are labelled by $k$ and $k^{\prime}$, with corresponding energies $E$ and $E^{\prime}$, and of the initial and final nucleons (mass $M$ ) by $p$ and $p^{\prime}$, respectively. The four-momentum transfer from the electron to the nucleon is given by $q=p^{\prime}-p=k-k^{\prime}$, with $Q^{2} \equiv-q^{2}>0$. One can express the elastic cross section in terms of any two of the Mandelstam variables $s$ (total electron-nucleon invariant mass squared), $t$, and $u$, where

$$
s=(k+p)^{2}=\left(k^{\prime}+p^{\prime}\right)^{2}, \quad t=\left(k-k^{\prime}\right)^{2}=q^{2}, \quad u=\left(p-k^{\prime}\right)^{2}=\left(p^{\prime}-k\right)^{2},
$$

with the constraint $s+t+u=2 M^{2}+2 m_{e}^{2}$. The electron mass $m_{e}$ can generally be ignored at the kinematics of interest here. In particular, there are no mass singularities in the limit $m_{e} \rightarrow 0$ in either the OPE amplitude or the total TPE amplitude.

Conventionally, the elastic scattering cross section is defined in terms of $Q^{2}$ and the electron scattering angle, $\theta_{e}$, or equivalently, any two of the dimensionless quantities

$$
\tau=\frac{Q^{2}}{4 M^{2}}, \quad \varepsilon=\frac{\nu^{2}-\tau(1+\tau)}{\nu^{2}+\tau(1+\tau)}=\frac{2\left(M^{4}-s u\right)}{s^{2}+u^{2}-2 M^{4}}, \quad \nu=\frac{k \cdot p}{M^{2}}-\tau .
$$

The inverse relationships are also useful:

$$
\nu=\frac{s-u}{4 M^{2}}=\sqrt{\frac{\tau(1+\tau)(1+\varepsilon)}{1-\varepsilon}} .
$$

In the target rest frame we have the relations

$$
\varepsilon=\left(1+2(1+\tau) \tan ^{2} \frac{\theta_{e}}{2}\right)^{-1}, \quad \tau=\frac{E-E^{\prime}}{2 M}, \quad \nu=\frac{E+E^{\prime}}{2 M},
$$

where $\varepsilon$ is identified with the relative flux of longitudinal virtual photons, and $E\left(E^{\prime}\right)$ is the energy of the incident (scattered) electron.

In the Born (OPE) approximation the electron-nucleon scattering invariant amplitude can be written as

$$
\mathcal{M}_{\gamma}=-\frac{e^{2}}{q^{2}} j_{\gamma \mu} J_{\gamma}^{\mu}
$$

where $e$ is the electric charge, and the matrix elements of the electromagnetic leptonic and hadronic currents are given in terms of the lepton $\left(u_{e}\right)$ and nucleon $\left(u_{N}\right)$ spinors by

$$
j_{\gamma \mu}=\bar{u}_{e}\left(k^{\prime}\right) \gamma_{\mu} u_{e}(k), \quad J_{\gamma}^{\mu}=\bar{u}_{N}\left(p^{\prime}\right) \Gamma_{\gamma}^{\mu}(q) u_{N}(p) .
$$



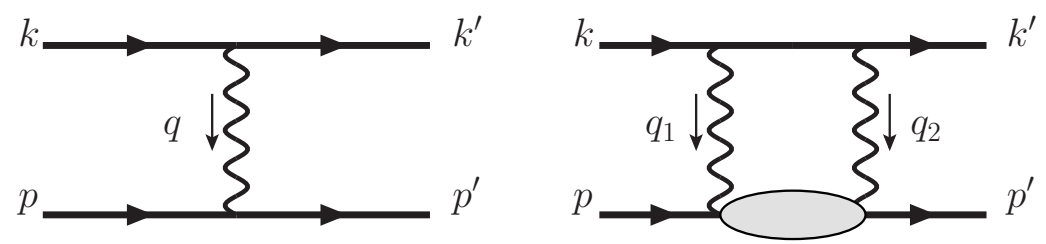

Figure 2.1: Contributions to elastic electron-nucleon scattering from (a) one-photon exchange (OPE), and (b) two-photon exchange (TPE) amplitudes, with particle momenta as indicated. For TPE we have $q_{1}+q_{2}=q$. Only the $s$-channel "box" diagram is drawn. The "crossed-box" contribution, which can be obtained by applying crossing symmetry $s \rightarrow u$, is implied.

The electromagnetic hadron current operator $\Gamma_{\gamma}^{\mu}$ is parametrized by the $\operatorname{Dirac}\left(F_{1}\right)$ and Pauli $\left(F_{2}\right)$ form factors as

$$
\Gamma_{\gamma}^{\mu}(q)=F_{1}\left(Q^{2}\right) \gamma^{\mu}+F_{2}\left(Q^{2}\right) \frac{i \sigma^{\mu \nu} q_{\nu}}{2 M} .
$$

In terms of the amplitude $\mathcal{M}_{\gamma}$, the differential Born cross section is given by

$$
\frac{d \sigma}{d \Omega}=\left(\frac{\alpha}{4 M Q^{2}} \frac{E^{\prime}}{E}\right)^{2}\left|\mathcal{M}_{\gamma}\right|^{2}=\frac{\sigma_{\mathrm{Mott}}}{\varepsilon(1+\tau)} \sigma_{R}, \quad \sigma_{\mathrm{Mott}}=\frac{\alpha^{2} E^{\prime} \cos ^{2}\left(\theta_{e} / 2\right)}{4 E^{3} \sin ^{4}\left(\theta_{e} / 2\right)},
$$

where $\alpha=e^{2} / 4 \pi$ is the electromagnetic fine structure constant, and the $\sigma_{\text {Mott }}$ is the cross section for scattering from a point particle. In our convention, the reduced Born cross section $\sigma_{R}$ is given by

$$
\sigma_{R}=\varepsilon G_{E}^{2}\left(Q^{2}\right)+\tau G_{M}^{2}\left(Q^{2}\right)
$$

where the Sachs electric and magnetic form factors $G_{E, M}\left(Q^{2}\right)$ are defined in terms of the Dirac and Pauli form factors as

$$
G_{E}\left(Q^{2}\right)=F_{1}\left(Q^{2}\right)-\tau F_{2}\left(Q^{2}\right), \quad G_{M}\left(Q^{2}\right)=F_{1}\left(Q^{2}\right)+F_{2}\left(Q^{2}\right) .
$$

The form factors are normalized such that $G_{E}^{p(n)}(0)=1(0)$ and $G_{M}^{p(n)}(0)=\mu_{p(n)}$ for the proton (neutron), where $\mu_{p(n)}=2.793(-1.913)$ is the proton (neutron) magnetic moment.

\subsection{Experimental measurements of proton form factors}

For many decades the standard experimental technique for extracting proton form factors has been the Rosenbluth, or longitudinal-transverse (LT), separation method [5]. The method requires applying a number of standard radiative corrections [6-8] to the measured cross section to extract a reduced cross section $\sigma_{R}$ equivalent to the OPE form given in Eq. (2.9). The standard radiative corrections are large, but generally speaking they are independent of hadron structure. Analyzing $\sigma_{R}$ as a function of the longitudinal photon polarization $\varepsilon$ at fixed $Q^{2}$ allows one to extract $G_{M}^{2}\left(Q^{2}\right)$ from the $\varepsilon$-intercept, and $G_{E}^{2}\left(Q^{2}\right)$ from the slope in $\varepsilon$. Because of the $\varepsilon / \tau$ weighting of $G_{E}^{2}$ relative to $G_{M}^{2}$, the contribution from the electric form factor to the cross section is suppressed at large $Q^{2}$. The proton form factor ratios extracted via the Rosenbluth technique have generally been consistent with $Q^{2}$ scaling $\left|G_{E}\right| \approx\left|G_{M} / \mu_{p}\right|[9-11]$.

An alternative method of extracting the form factors, known as the polarization transfer (PT) technique $[12,13]$, utilizes polarization degrees of freedom to increase the sensitivity to the electric form factor at large $Q^{2}$. Here, longitudinally polarized electrons are scattered from an unpolarized proton target, with the polarization of the recoiling proton detected, $\vec{e}+p \rightarrow e+\vec{p}$. 


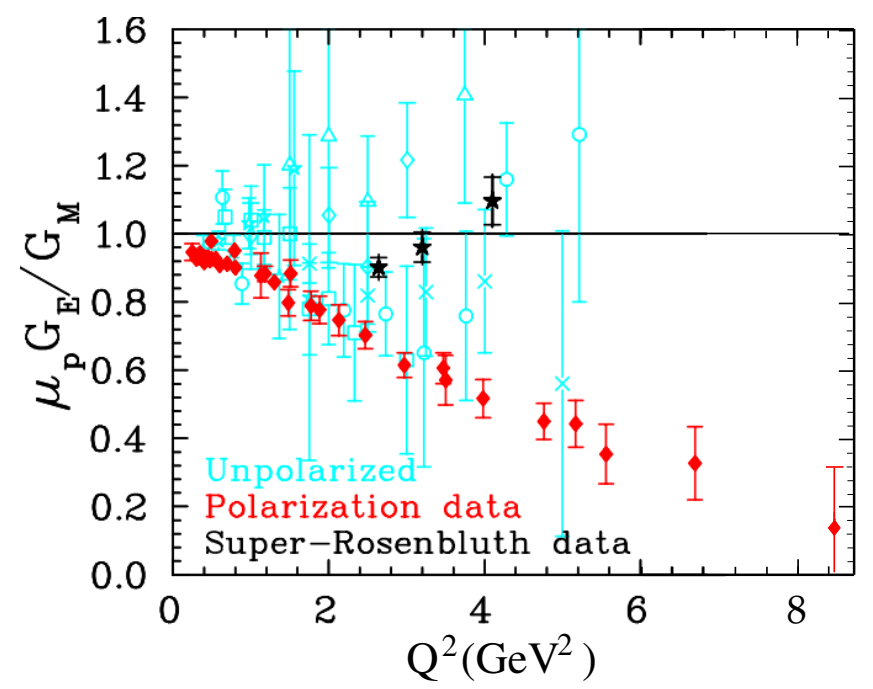

Figure 2.2: Ratio $R=\mu_{p} G_{E}^{p} / G_{M}^{p}$ as extracted using Rosenbluth separation [14] (open cyan points), polarization transfer measurements [15-19] (filled red diamonds), and "Super-Rosenbluth" measurements [11] (black stars). Figure taken from Ref. [20].

In the Born approximation the elastic cross section for scattering a longitudinally polarized electron of helicity $h$ with a recoil proton polarized longitudinally (i.e. in the direction of motion) is given by

$$
\frac{d \sigma^{(L)}}{d \Omega}=h \sigma_{\mathrm{Mott}} \frac{E+E^{\prime}}{M} \sqrt{\frac{\tau}{1+\tau}} \tan ^{2} \frac{\theta_{e}}{2} G_{M}^{2} .
$$

For a recoil proton detected with a polarization transverse to the proton momentum, but still in the scattering plane, the cross section is

$$
\frac{d \sigma^{(T)}}{d \Omega}=h \sigma_{\mathrm{Mott}} 2 \sqrt{\frac{\tau}{1+\tau}} \tan \frac{\theta_{e}}{2} G_{E} G_{M} .
$$

Taking the ratio of the transverse to longitudinal proton cross sections then yields the ratio of the electric to magnetic proton form factors,

$$
R=-\mu_{p} \sqrt{\frac{\tau(1+\varepsilon)}{2 \varepsilon}} \frac{P_{T}}{P_{L}}=-\mu_{p} \frac{E+E^{\prime}}{2 M} \tan \frac{\theta_{e}}{2} \frac{P_{T}}{P_{L}}=\mu_{p} \frac{G_{E}}{G_{M}},
$$

where $P_{L}$ and $P_{T}$ are the polarizations of the recoil proton longitudinal and transverse to the proton momentum in the scattering plane, respectively. The polarization transfer normal to the scattering plane, $P_{N}$, vanishes in the OPE approximation, but it can be non-zero in general.

In a series of experiments at Jefferson Lab [15-19, 21-25] beginning in the late 1990's, the polarization transfer (PT) technique was used to accurately determine the ratio $G_{E} / G_{M}$ up to $Q^{2}=8.5 \mathrm{GeV}^{2}$. In addition, there have been complementary measurements using polarized targets at MIT-Bates [26] and Jefferson Lab [27]. The results, illustrated in Fig. 2.2, are in striking contrast to the ratio obtained via LT or Rosenbluth separations, showing an approximately linear decrease of $R$ with $Q^{2}$.

The discrepancy between the LT and PT measurements of $G_{E} / G_{M}$ has stimulated considerable theoretical and experimental activity over the past 15 years. Attempts to reconcile the measurements have mostly focussed on improved treatments of radiative corrections, particularly those associated with the model-dependent hadronic terms that arise in TPE. These terms can lead to additional $\varepsilon$-dependence of the cross section. In the following sections we discuss theoretical efforts to better understand the discrepancy, as well as the impact of these calculations on other observables (e.g. single-spin asymmetries). 


\subsection{Two-photon exchange}

The first quantitative calculation to address the $G_{E} / G_{M}$ discrepancy was made by Blunden et al. [28], who computed the effect on $G_{E} / G_{M}$ from TPE, incorporating explicitly the nucleon's substructure through hadronic form factors. A number of other studies have followed, examining TPE in a variety of frameworks, and exploring reactions beyond elastic ep scattering. In a parallel effort, Guichon and Vanderhaeghen [29] provided a generalized formalism for elastic scattering, allowing for possible TPE contributions through three "generalized form factors" denoted as $\tilde{F}_{1}$, $\tilde{F}_{2}$, and $\tilde{F}_{3}$. They demonstrated that it was natural to have TPE contributions that could significantly change the LT extraction of $G_{E} / G_{M}$ with minimal impact on the PT measurements. In this section we review these efforts, paying particular attention to recent progress in conventional hadronic-level calculations, which are most applicable to data analysis at low to moderate $Q^{2}$ values. Theoretical progress on TPE calculations at higher $Q^{2}$, using partonic degrees of freedom, is briefly discussed in the final subsection.

\subsubsection{General formulation}

Using the kinematics of Fig. 2.1, the contribution to the TPE box amplitude from an intermediate hadronic state $R$ of invariant mass $M_{R}$ can be written in the general form $[28,30]$

$$
\mathcal{M}_{\gamma \gamma}^{\text {box }}=-i e^{4} \int \frac{d^{4} q_{1}}{(2 \pi)^{4}} \frac{L_{\mu \nu} H_{R}^{\mu \nu}}{\left(q_{1}^{2}-\lambda^{2}\right)\left(q_{2}^{2}-\lambda^{2}\right)},
$$

with $q_{2}=q-q_{1}$, and an infinitesimal photon mass $\lambda$ is introduced to regulate any infrared divergences. The leptonic and hadronic tensors are given by

$$
\begin{aligned}
L_{\mu \nu} & =\bar{u}_{e}\left(k^{\prime}\right) \gamma_{\mu} S_{F}\left(k-q_{1}, m_{e}\right) \gamma_{\nu} u_{e}(k), \\
H^{\mu \nu} & =\bar{u}_{N}\left(p^{\prime}\right) \Gamma_{R \rightarrow \gamma N}^{\mu \alpha}\left(p+q_{1},-q_{2}\right) S_{\alpha \beta}\left(p+q_{1}, M_{R}\right) \Gamma_{\gamma N \rightarrow R}^{\beta \nu}\left(p+q_{1}, q_{1}\right) u_{N}(p) .
\end{aligned}
$$

The electron propagator is

$$
S_{F}\left(k, m_{e}\right)=\frac{\left(\not k+m_{e}\right)}{k^{2}-m_{e}^{2}+i \epsilon} .
$$

The hadronic transition current operator $\gamma N \rightarrow R$ is written in a general form $\Gamma_{\gamma N \rightarrow R}^{\alpha \mu}\left(p_{R}, q\right)$ that allows for a possible dependence on the incoming momentum $q$ of the photon and the outgoing momentum $p_{R}$ of the hadron, while $\mu$ and $\alpha$ are Lorentz indices, and $S_{\alpha \beta}\left(p_{R}, M_{R}\right)$ is the hadronic state propagator. One can obtain the crossed-box term directly from the box term by applying crossing symmetry. For example, in the unpolarized case, we have

$$
\mathcal{M}_{\gamma \gamma}^{\mathrm{xbox}}(u, t)=-\left.\mathcal{M}_{\gamma \gamma}^{\mathrm{box}}(s, t)\right|_{s \rightarrow u} .
$$

In general, $\mathcal{M}_{\gamma \gamma}^{\text {box }}(s, t)$ has both real and imaginary parts, whereas $\mathcal{M}_{\gamma \gamma}^{\text {xbox }}(u, t)$ is purely real. The imaginary part is of interest for calculations of the normal spin asymmetry, which is discussed in section (2.4) of this Review. The imaginary part is also useful as an alternative method to calculating the real part through the use of dispersion relations and analyticity. This is discussed in further detail in section (2.3.4).

The relative correction to the reduced Born cross section, Eq. (2.9), due to the interference of the one- and two-photon exchange amplitudes shown in Fig. 2.1, is given by

$$
\delta_{\mathrm{TPE}}=\frac{2 \operatorname{Re}\left(\mathcal{M}_{\gamma}^{*} \mathcal{M}_{\gamma \gamma}\right)}{\left|\mathcal{M}_{\gamma}\right|^{2}} .
$$

Within the framework of the simplest hadronic models, analytic evaluation of $\delta_{\text {TPE }}$ is made possible by writing the transition form factors at the $\gamma$-hadron vertices as a sum and/or product of monopole form factors [28, 31], which are typically fit to empirical transition form factors over a suitable range in space-like four-momentum transfer. Four-dimensional integrals over the momentum in the one-loop box diagram can then be expressed in terms of Passarino-Veltman scalar functions $A_{0}, B_{0}, C_{0}$, and $D_{0}[32,33]$, which can be evaluated numerically using packages like LoopTools [34]. 


\subsubsection{Model-independent TPE corrections}

Expression (2.19) contains infrared (IR) divergences arising from the elastic intermediate state when the momentum $q_{i}$ of either photon goes to 0. In analyzing the TPE corrections for ep scattering, it is convenient to separate terms into the "soft" parts, which are independent of hadronic structure, and the "hard" parts, which are model-dependent. Soft here implies that the interaction of one of the virtual photon with the proton occurs with vanishingly small momentum transfer. As the soft parts are independent of hadron structure, they are therefore universal, i.e. the same for protons as they are for scattering from point-like particles. All of the IR divergences for the virtual diagrams are contained in the soft parts, and these divergences cancel in the total amplitude when added to the inelastic bremsstrahlung contributions involving the emission of a real, soft photon.

Because the soft, IR-divergent part of the TPE amplitude is already included with other modelindependent radiative corrections in experimental analyses, it has become conventional to consider only the hard part of the TPE amplitude in discussions of TPE effects. This can be accomplished by an appropriate subtraction of a conventionally defined soft part from the full TPE amplitude. Two conventions in common use for the soft, model-independent part of the TPE amplitude are those of Mo and Tsai [6, 7], and Maximon and Tjon [8]. These are discussed extensively in a previous TPE review by Arrington, Blunden, and Melnitchouk [2], and we defer to that paper for details. The explicit expressions are:

$$
\begin{aligned}
\delta_{\mathrm{IR}}(\mathrm{MoT}) & =-\frac{2 \alpha}{\pi}\left[\log \eta \log \left(\frac{2 M \sqrt{E E^{\prime}}}{\lambda^{2}}\right)-\mathrm{Li}_{2}\left(1-\frac{M}{2 E}\right)+\operatorname{Li}_{2}\left(1-\frac{M}{2 E^{\prime}}\right)\right], \\
\delta_{\mathrm{IR}}(\mathrm{MTj}) & =-\frac{2 \alpha}{\pi} \log \eta \log \frac{Q^{2}}{\lambda^{2}}
\end{aligned}
$$

with $\eta=E / E^{\prime}$ the ratio of incident to final electron energies, and $\mathrm{Li}_{2}$ is the dilogarithm function. For historical reasons, the Mo-Tsai expression is the one generally used in existing experimental computer codes. However, the Maximon-Tjon expression has also been used in more recent experimental analyses [35]. A meaningful comparison to data can therefore be made by considering the difference

$$
\delta_{\gamma \gamma} \equiv \delta_{\mathrm{TPE}}-\delta_{\mathrm{IR}}(\mathrm{MoT})
$$

for which the IR divergences cancel, and is therefore independent of $\lambda$. Here $\delta_{\gamma \gamma}$ represents TPE effects that are unaccounted for after applying the standard radiative corrections to data. The measured reduced cross section $\sigma_{R}^{\text {meas }}$ is therefore related to $\sigma_{R}$ by

$$
\sigma_{R}^{\text {meas }}=\sigma_{R}\left(1+\delta_{\gamma \gamma}\right)
$$

Therefore a positive slope for $\delta_{\gamma \gamma}$ versus $\varepsilon$ means that the form factor $G_{E}^{p}$ inferred from $\sigma_{R}^{\text {meas }}$ is larger than its actual value, which is what the PT data is telling us.

\subsubsection{One-loop methods}

The box (plus crossed-box) TPE contributions were evaluated by Blunden and collaborators in a series of papers using one-loop integration techniques. Intermediate states they considered include elastic $(N)[28,31]$ and inelastic $\Delta$ excitations [30] evaluated within a hadronic framework. The contribution of the most important heavier spin- $1 / 2$ and spin- $3 / 2$ resonances was also estimated [36], using nucleon Compton scattering calculations at low energies as input parameters. In addition, both electromagnetic $\gamma \gamma$ and electroweak $\gamma Z$ box contributions to parity-violating electron scattering have been calculated within the same framework [37-40]. The key findings of these investigations were discussed in the Review by Arrington et al. [2]. In this section we briefly summarize these results, and then discuss recent advances, particularly in the contribution of excited intermediate states, before turning to the approach of using dispersion relations. 

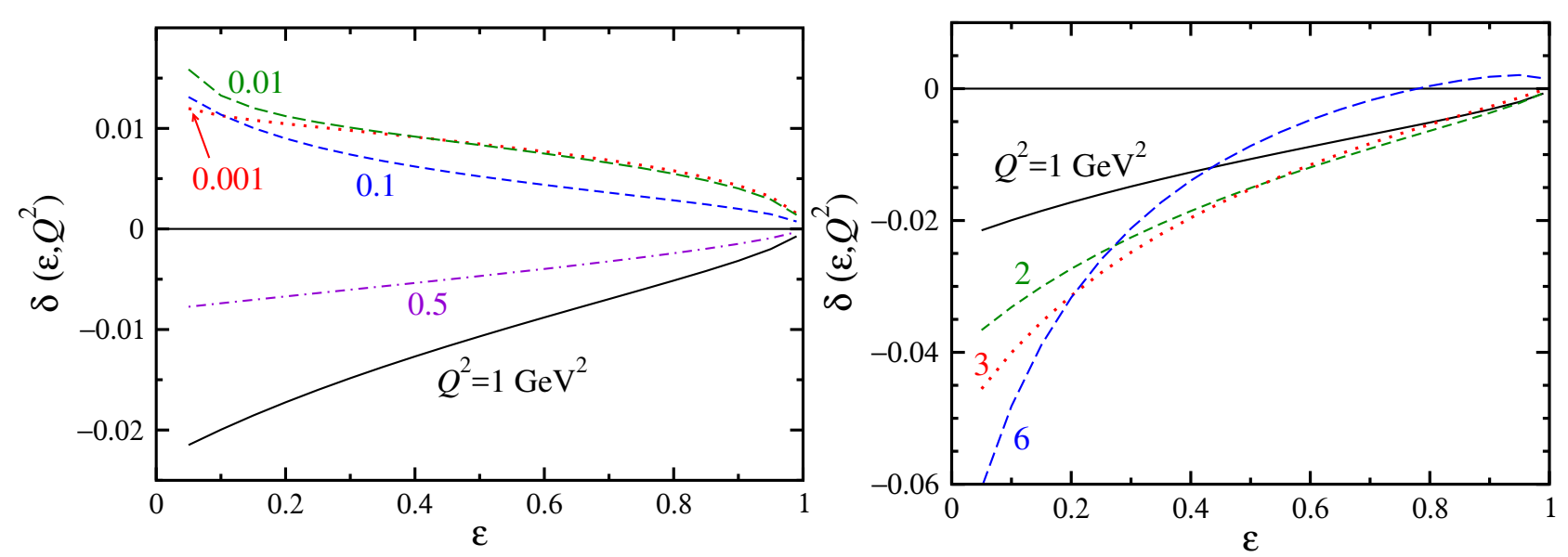

Figure 2.3: TPE correction of Eq. (2.22) to elastic ep scattering for intermediate nucleon states [28]. Curves are for $Q^{2}=0.001-1 \mathrm{GeV}^{2}$ (left), and $Q^{2}=1-6 \mathrm{GeV}^{2}$ (right).

Elastic contribution. The elastic contribution, $\delta_{N}$, dominates the TPE corrections at low to moderate $Q^{2}$. Results for $\delta_{N}$ relative to the Mo-Tsai approximation are shown in Fig. 2.3 as a function of $\varepsilon$ for several values of $Q^{2}$, from 0.001 to $1 \mathrm{GeV}^{2}$ (left panel) and 1 to $6 \mathrm{GeV}^{2}$ (right panel). These curves use the form factor parametrization of Arrington et al. [41], although in practice the results are fairly insensitive to the details of the form factors.

The hadron structure-dependent corrections are most significant at small $\varepsilon$, where they range from $\sim+1.5 \%$ at low $Q^{2}$ to $\sim-6 \%$ at $Q^{2}=6 \mathrm{GeV}^{2}$. At high $Q^{2}$ the magnetic form factor $G_{M}$ dominates in the loop integrals. The TPE effect vanishes as $\varepsilon \rightarrow 1$, a requirement linked to unitarity. As noted by Blunden et al. [28], the positive slope in $\varepsilon$ at high $Q^{2}$ is of the right sign and magnitude to explain the observed ratio $G_{E} / G_{M}$. At lower $Q^{2}$ values, $\delta_{N}$ is approximately linear in $\varepsilon$, but significant deviations from linearity are observed with increasing $Q^{2}$, especially at small $\varepsilon$. For $Q^{2} \lesssim 0.3 \mathrm{GeV}^{2}, \delta_{N}$ becomes positive, and the electric form factor $G_{E}$ dominates in the loop integrals.

At very low $Q^{2} \lesssim 0.01 \mathrm{GeV}^{2}, \delta_{N}$ approaches the static limit for a structureless, massive target,

$$
\delta_{N} \underset{Q^{2} \rightarrow 0}{\longrightarrow} \frac{\alpha \pi}{x+1}, \quad x=\sqrt{\frac{1+\varepsilon}{1-\varepsilon}} .
$$

This result was first derived in the second Born approximation by McKinley and Feshbach [42], who expressed it in terms of $\sin \left(\theta_{e} / 2\right)=1 / x$.

Inelastic contributions. In view of the prominent role of the $\Delta(1232)$ resonance in the electromagnetic excitation spectrum of the nucleon, it is important to evaluate its contribution to the TPE amplitude. The $\gamma N \rightarrow \Delta$ electromagnetic transition can be expressed in terms of three Jones-Scadron transition form factors, $G_{M}^{*}\left(Q^{2}\right), G_{E}^{*}\left(Q^{2}\right)$, and $G_{C}^{*}\left(Q^{2}\right)$, corresponding to magnetic, electric, and Coulomb multipole excitations, respectively. The magnetic multipole dominates in this transition. Although the $\gamma N \rightarrow \Delta$ cross section is diagonal in these functions, they are cumbersome to work with in the transition vertex function. For that purpose, an on-shell equivalent parametrization of the $\gamma N \rightarrow \Delta$ vertex is [30]

$$
\begin{aligned}
\Gamma_{\gamma N \rightarrow \Delta}^{\alpha \mu}\left(p_{\Delta}, q\right)= & \frac{1}{2 M_{\Delta}^{2}} \sqrt{\frac{2}{3}}\left\{g_{1}\left(Q^{2}\right)\left[g^{\alpha \mu} \not \not p_{\Delta}-\not q \gamma^{\alpha} p_{\Delta}^{\mu}-\gamma^{\alpha} \gamma^{\mu} q \cdot p_{\Delta}+\not p_{\Delta} \gamma^{\mu} q^{\alpha}\right]\right. \\
& +g_{2}\left(Q^{2}\right)\left[q^{\alpha} p_{\Delta}^{\mu}-g^{\alpha \mu} q \cdot p_{\Delta}\right] \\
& \left.+\frac{g_{3}\left(Q^{2}\right)}{M_{\Delta}}\left[q^{2}\left(\gamma^{\alpha} p_{\Delta}^{\mu}-g^{\alpha \mu} \not p_{\Delta}\right)+q^{\mu}\left(q^{\alpha} \not p_{\Delta}-\gamma^{\alpha} q \cdot p_{\Delta}\right)\right]\right\} \gamma_{5},
\end{aligned}
$$




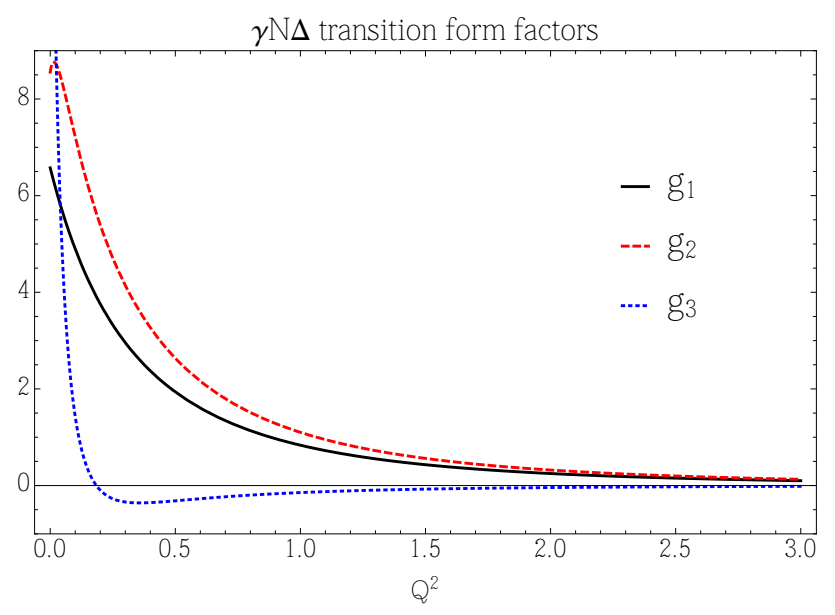

Figure 2.4: The $\gamma N \rightarrow \Delta$ transition form factors based on a fit of Ref. [43]. Over this range of $Q^{2}, g_{1}\left(Q^{2}\right)$ is well approximated by a dipole form factor with mass parameter $\Lambda_{\Delta}=0.76 \mathrm{GeV}$.

where $p_{\Delta}$ and $q$ are the momenta of the outgoing $\Delta$ and incoming photon. A parametrization of all three $g_{i}\left(Q^{2}\right)$ transition form factors, based on recent fits to electro-production data by Aznauryan and Burkert [43], is shown in Fig. 2.4. Briefly, $g_{1}$ is determined by the dominant $G_{M}^{*}$ magnetic form factor, $\left(g_{2}-g_{1}\right)$ is primarily sensitive to the electric form factor $G_{E}^{*}$, and $g_{3}$ is sensitive to the Coulomb form factor $G_{C}^{*}$. Over the range $0<Q^{2}<3 \mathrm{GeV}^{2}$, the $g_{1}$ form factor is well approximated by a dipole, with mass parameter $\Lambda_{\Delta}=0.76 \mathrm{GeV}$.

In Ref. [30] the $g_{1}$ and $g_{2}$ form factors were assumed to have dipole shapes, $g_{i}\left(Q^{2}\right)=g_{i}(0) /(1+$ $\left.Q^{2} / \Lambda_{\Delta}^{2}\right)^{2}$, with a dipole mass $\Lambda_{\Delta}$ ranging between hard $\left(\Lambda_{\Delta}=0.84 \mathrm{GeV}\right)$ and soft $\left(\Lambda_{\Delta}=0.69 \mathrm{GeV}\right)$ to gauge the sensitivity of their shape on contribution of the $\Delta$ to TPE. The $g_{3}$ form factor was set to 0 . No attempt was made to make a detailed fit of these form factors to available data. To summarize the key findings of these early investigations: The TPE $\Delta$ intermediate state contribution is smaller and of opposite sign to the $N$ one, thereby attenuating the $N$ contribution somewhat; it increases in magnitude as $Q^{2}$ increases; and it diverges as $\varepsilon \rightarrow 1$, in apparent violation of the unitarity constraint. This divergence is most apparent for $\varepsilon \gtrsim 0.9$, and is sensitive to the transition form factor (soft or hard). These features are also found in the calculations of Refs. [38-40].

Recent work. More recently, Zhou and Yang [44], Graczyk [45], and Lorenz et al. [46] improved on this early work, primarily through the use of $\gamma N \Delta$ transition form factors with a closer fit to data.

Zhou and Yang [44] improved the treatment of the $\gamma N \Delta$ transition form factors by fitting a sum of monopoles (or dipoles) to existing electroproduction data. They also included all three form factors: $g_{1}, g_{2}$, and $g_{3}$. Figure 2.5 shows the effect on $\delta_{\Delta}$ in comparison to the simpler parametrization of Kondratyuk et al. [30]. The overall contribution with realistic form factors is somewhat smaller than the hard-dipole fit of Ref. [30]. The effect of including the Coulomb contribution (arising from $g_{3}$ ) is found to be small.

Graczyk [45] obtained predictions for the TPE correction to the unpolarized ep elastic cross section in two different approaches. The first is a standard calculation using one-loop box diagrams with $N$ and $\Delta\left(P_{33}\right.$ resonance $)$ as hadronic intermediate states. Different form factor parametrizations of the $\gamma N \Delta$ transition form factors were taken into consideration. In the second approach the phenomenological TPE correction was extracted from experimental data by applying the Bayesian neural network (BNN) statistical framework. The BNN response was constrained by assuming that the PT data are not sensitive to TPE effects. Predictions of the two methods agree well in the intermediate $Q^{2}$ range of 1-3 $\mathrm{GeV}^{2}$, and agree at the $2 \sigma$ level above this range. Below $Q^{2}=1 \mathrm{GeV}^{2}$ the two methods disagree (see Fig. 2.6). The effect on the ratio $G_{E} / G_{M}$ of the combined $N+\Delta$ TPE contributions, as evaluated 


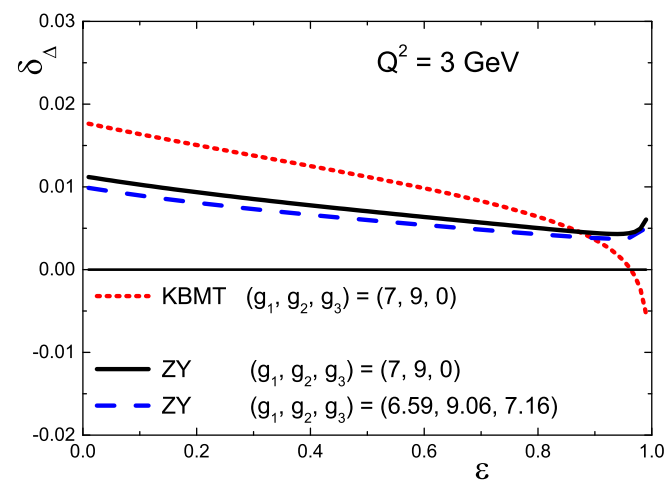

Figure 2.5: Contribution of $\delta_{\Delta}$ at $Q^{2}=3 \mathrm{GeV}^{2}$, adapted from Zhou and Yang [44]. The dotted curve is based on a calculation by Kondratyuk et al. [30], and uses magnetic and electric form factors of dipole form $\left(\Lambda_{\Delta}=0.84 \mathrm{GeV}\right)$. The solid curve uses the same coupling strengths but with a realistic form factor shape fit to data [44], while the long-dashed curve shows the effect of using all three transition form factors with coupling strengths and shapes fit to data [44].
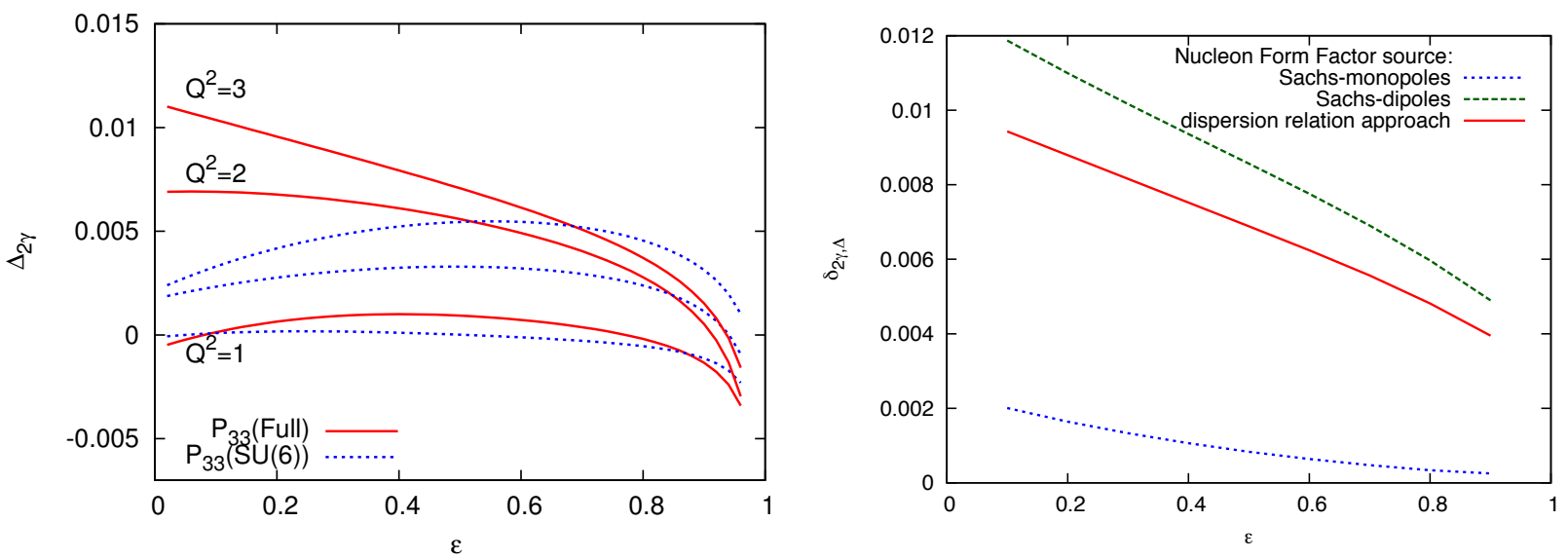

Figure 2.6: Left: The $\Delta$ TPE contribution $\delta_{\Delta}$ from Graczyk [45], showing the effect of different $\gamma N \Delta$ form factor parametrizations for three values of $Q^{2}$. Right: $\delta_{\Delta}$ at $Q^{2}=3 \mathrm{GeV}^{2}$ from Lorenz et al. [46], showing the effect of different transition form factor models.

by Graczyk [45], is shown in Fig. 2.7. There is reasonably good agreement with data over the range of $Q^{2}$ given.

Lorenz et al. [46] also evaluated the $\Delta$ contribution to the TPE amplitude, using $\gamma N \Delta$ transition vertices matched to helicity amplitudes from electroproduction of nucleon resonances. They also considered the effect of nucleon form factors fits constrained by analyticity and unitarity, denoted as "dispersion relation" in Fig. 2.6. Their results are in good agreement with those of Refs. [44, 45].

Despite the improved fits of the transition form factors in Refs. [44-46] the divergence of the $\Delta$ contribution as $\varepsilon \rightarrow 1$ that was seen in earlier work [30, 38-40] is still apparent.

In general the contributions of all the heavier resonances are much smaller than those of the nucleon and $\Delta\left(P_{33}\right)[36]$. However, there is an interesting interplay between the contributions of the spin$1 / 2$ and spin- $3 / 2$ resonances, which is analogous to the partial cancellation of the two-photon exchange effects of the nucleon and $\Delta$ intermediate states, found in Ref. [30]. Notwithstanding the smallness of the resonance contributions, their inclusion in the TPE diagrams leads to a better agreement between the Rosenbluth and polarization transfer data analyses, especially at higher values of the momentumtransfer squared $Q^{2}$.

Inclusion of contributions of intermediate states with masses larger than $\sim 2 \mathrm{GeV}$ becomes imprac- 

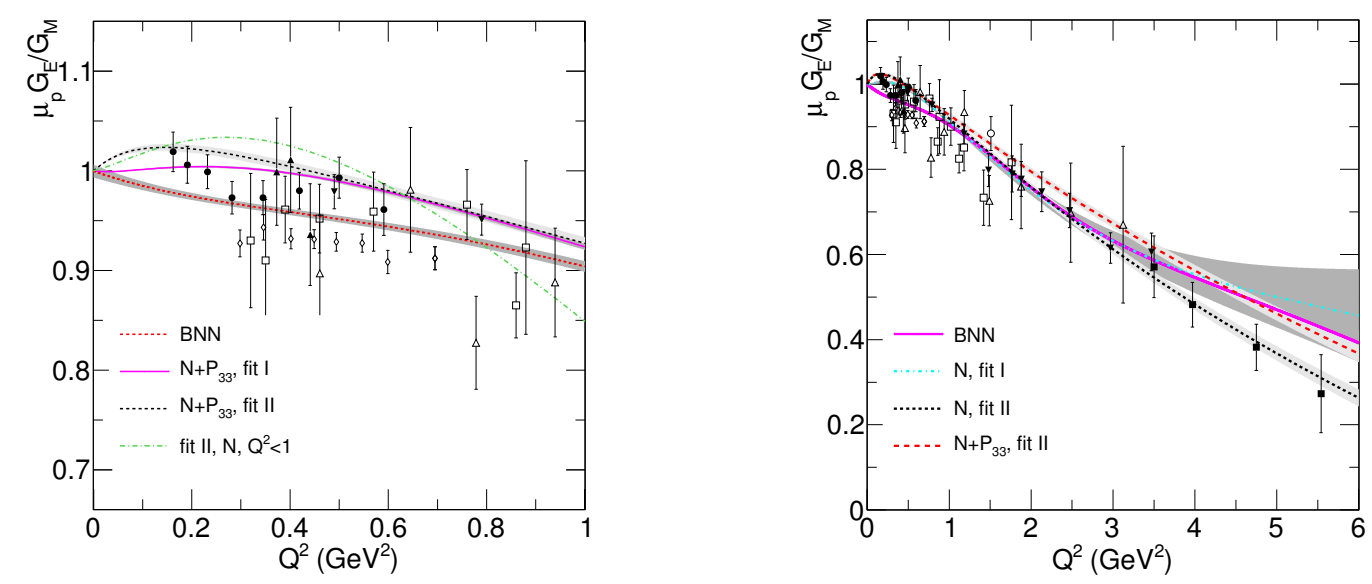

Figure 2.7: The ratio $R=\mu_{p} G_{E} / G_{M}$ calculated by TPE fits using intermediate states $N$ only and $N+P_{33}$, as well as the BNN fit. The shaded area shows the $1 \sigma$ error from the fit. The ratio $R$ at low $Q^{2}($ left $)$ and at high $Q^{2}($ right). Figures taken from Gracyzk [45].

tical within a hadronic approach when one moves beyond the resonance region. Here it becomes more efficient to use partonic degrees of freedom [47-50]. These were discussed in the Review by Arrington et al. [2]. We give a brief summary of these contributions in Sec. 2.3.5.

\subsubsection{Dispersive methods}

As noted previously, $\mathcal{M}_{\gamma \gamma}$ of Eq. (2.14) has both real and imaginary parts. The imaginary parts come from the box diagram, and are completely determined by terms in the amplitude where the electron and hadron intermediate states are on-shell. In the loop integral method, the imaginary parts are completely contained in the Passarino-Veltman functions. The real and imaginary parts are related by dispersion relations [51, 52], which forms the basis of the dispersive method discussed in this section.

Following the formalism introduced by Guichon and Vanderhaeghen [29], $\mathcal{M}_{\gamma \gamma}$ can be mapped onto three generalized form factors $\tilde{F}_{i}\left(Q^{2}, \nu\right)$ :

$$
\begin{aligned}
\mathcal{M}_{\gamma \gamma} \longrightarrow & -\frac{e^{2}}{q^{2}} \bar{u}_{e}\left(k^{\prime}\right) \gamma_{\mu} u_{e}(k) \times \\
& \bar{u}_{N}\left(p^{\prime}\right)\left[\tilde{F}_{1}\left(Q^{2}, \nu\right) \gamma^{\mu}+\tilde{F}_{2}\left(Q^{2}, \nu\right) \frac{i \sigma^{\mu \nu} q_{\nu}}{2 M}+\tilde{F}_{3}\left(Q^{2}, \nu\right) \frac{\not k P^{\mu}}{M^{2}}\right] u_{N}(p),
\end{aligned}
$$

with $K=\left(k^{\prime}+k\right) / 2$ and $P=\left(p^{\prime}+p\right) / 2$. Elastic electron scattering observables (including polarization transfer) can be expressed in terms of these generalized form factors [29]. To calculate their imaginary parts explicitly, we can put the intermediate electron and hadron on-shell in Eq. (2.14), thus reducing the four-dimensional loop integral to a two-dimensional integral over $Q_{1}^{2}$ and $Q_{2}^{2}$ - the four-momentum squared of each virtual photon.

The generalized form factors satisfy fixed- $t$ dispersion relations [51]

$$
\begin{aligned}
\operatorname{Re} \tilde{F}_{1}\left(Q^{2}, \nu\right) & =\frac{2}{\pi} \mathcal{P} \int_{\nu_{\text {th }}}^{\infty} d \nu^{\prime} \frac{\nu}{\nu^{\prime 2}-\nu^{2}} \operatorname{Im} \tilde{F}_{1}\left(Q^{2}, \nu^{\prime}\right), \\
\operatorname{Re} \tilde{F}_{2}\left(Q^{2}, \nu\right) & =\frac{2}{\pi} \mathcal{P} \int_{\nu_{\text {th }}}^{\infty} d \nu^{\prime} \frac{\nu}{\nu^{\prime 2}-\nu^{2}} \operatorname{Im} \tilde{F}_{2}\left(Q^{2}, \nu^{\prime}\right), \\
\operatorname{Re} \tilde{F}_{3}\left(Q^{2}, \nu\right) & =\frac{2}{\pi} \mathcal{P} \int_{\nu_{\text {th }}}^{\infty} d \nu^{\prime} \frac{\nu^{\prime}}{\nu^{\prime 2}-\nu^{2}} \operatorname{Im} \tilde{F}_{3}\left(Q^{2}, \nu^{\prime}\right),
\end{aligned}
$$

where $\mathcal{P}$ denotes the Cauchy principal value integral, and $\nu_{\mathrm{th}}=-\tau$. This extends the integral into the unphysical region $\cos \theta_{e}<-1$, which requires knowledge of the transition form factors in the timelike 

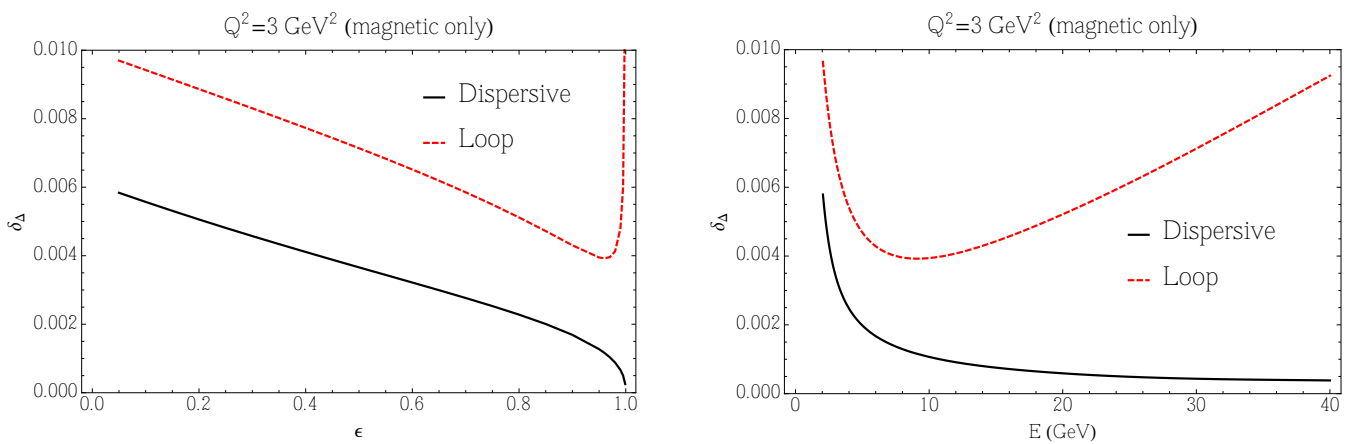

Figure 2.8: A comparison of $\delta_{\Delta}$ calculated using the loop integration method with that calculated in the dispersive method. Plotted is the result at $Q^{2}=3 \mathrm{GeV}^{2}$ for magnetic $\gamma N \Delta$ transition only, using a realistic transition form factor. The left figure shows $\delta_{\Delta}$ plotted against $\varepsilon$. The right figure shows same result plotted against electron energy $E$, where the linear divergence with $E$ becomes apparent. Figure adapted from Blunden and Melnitchouk [54].

region. For the interaction of point particles, such as in $e \mu$ scattering, the real parts generated in this way agree completely with those obtained directly from the four-dimensional loop integrals of Eq. (2.14) [53].

However, this equality no longer holds when the transition current operator depends on the momentum of the hadron, as it does for the $\gamma N \Delta$ vertex of Eq. (2.25) [54]. As a specific example, the left plot in Fig. 2.8 compares the TPE correction $\delta_{\Delta}$ calculated using the dispersive method (solid curve) with the correction calculated using loop integration (dashed curve), plotted as a function of $\varepsilon$ at $Q^{2}=3 \mathrm{GeV}^{2}[54]$. Only the dominant magnetic transition is considered here (by setting $g_{1}=g_{2}$ and $g_{3}=0$ in Eq. (2.25)), and the realistic form factor parametrization shown in Fig. 2.4 [43] is used for $g_{1}\left(Q^{2}\right)$. The imaginary parts of $\delta_{\Delta}$ (not shown) are identical, but the real parts (as plotted) differ significantly. The dispersive result vanishes as $\varepsilon \rightarrow 1$, and it is smaller in magnitude over the whole range of $\varepsilon$. The high- $\varepsilon$ behaviour can be seen more easily in the right plot, where $\delta_{\Delta}$ is plotted against electron energy $E$. The limit $\varepsilon \rightarrow 1$ corresponds to $E \rightarrow \infty$ at fixed $Q^{2}$. The divergence in the loop integral method is linear in $E$ due to the unconstrained linear factors of hadron momentum $p$ at each $\gamma N \Delta$ vertex. By contrast, the dispersive curve falls off like $1 / E$. A linear growth in $E$ exceeds the Froissart bound [55], and signals a violation of unitarity due to the unphysical off-shell behaviour of the transition current operators. Including the electric and Coulomb contributions does not alter these behaviours [54]. Thus while the loop integration method gives qualitatively the right behaviour in $\delta_{\Delta}$ for $\varepsilon \lesssim 0.9$, it is likely unreliable for precision comparisons with experimental data.

Recent work. Borisyuk and Kobushkin $[52,56]$ evaluated both $N$ and $\Delta$ contributions using the dispersive method. Empirical $\gamma N \Delta$ transition form factors were fit to a sum of monopoles parametrization to allow for analytic evaluation. More recently, they have extended their model to include the effect of $\pi N$ intermediate hadronic states, concentrating on the $P_{33}$ [57], and seven other spin- $1 / 2$ and spin- $3 / 2$ channels [58]. Their calculation includes a resonance width and shape, as well as background contributions. A sample of their calculations of the TPE contributions to $\delta_{\gamma \gamma}$ from different $\pi N$ channels at $Q^{2}=1 \mathrm{GeV}^{2}$ and $5 \mathrm{GeV}^{2}$ is shown in Fig. 2.9. As expected, the $P_{33}$ channel dominates, but substantial contributions from $P_{11}$ and $S_{11}$ channels are also present. The inelastic contributions are small at low $Q^{2}$, and all vanish in the limit $\varepsilon \rightarrow 1$.

Tomalak and Vanderhaeghen [59] also used the dispersive method to evaluate TPE effects for $N$ intermediate states. They used a subtracted dispersion relation to improve the fits to observables. This paper introduces a contour integration method to evaluate the two-dimensional loop integrals needed for the imaginary part of the generalized form factors in the unphysical region $(-1<\varepsilon<0)$.

Blunden and Melnitchouk [54] evaluated both $N$ and $\Delta$ contributions using the dispersive method, with realistic $\gamma N N$ and $\gamma N \Delta$ transition form factors taken from electro-production data. By using numerical contour integration in the unphysical region, based on the approach of Tomalak and Vander- 

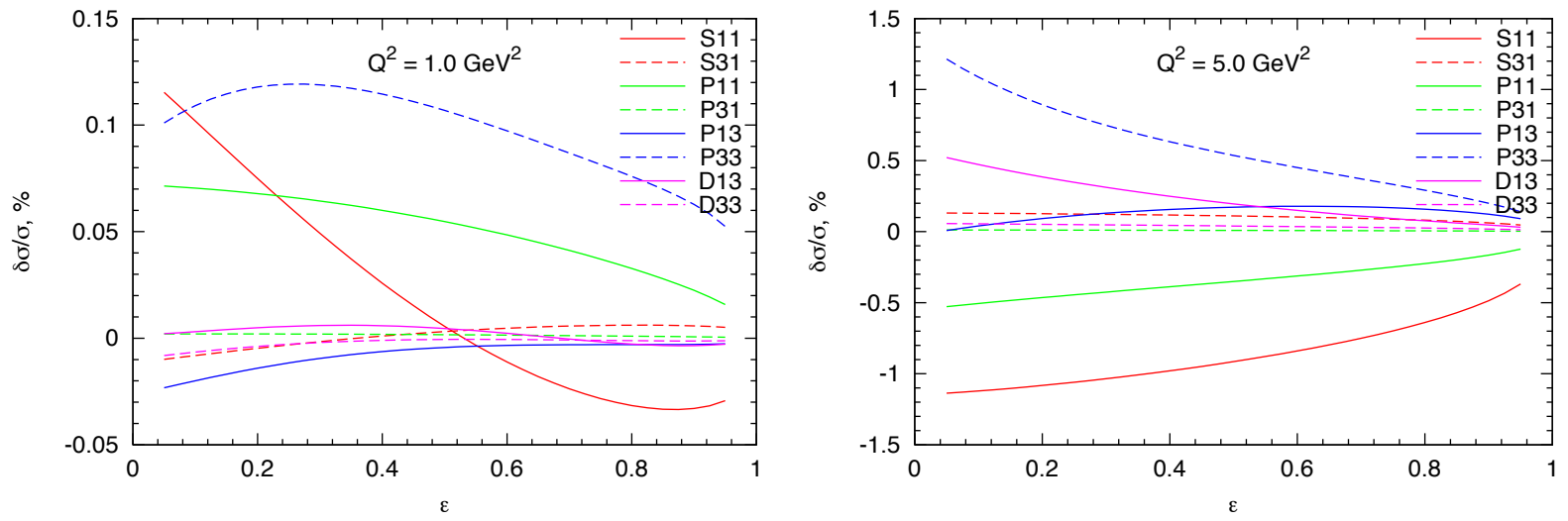

Figure 2.9: TPE contributions to $\delta_{\gamma \gamma}$ from different $\pi N$ channels at two different values of $Q^{2}$. Note the different scales. Figure taken from Borisyuk and Kobushkin [58].
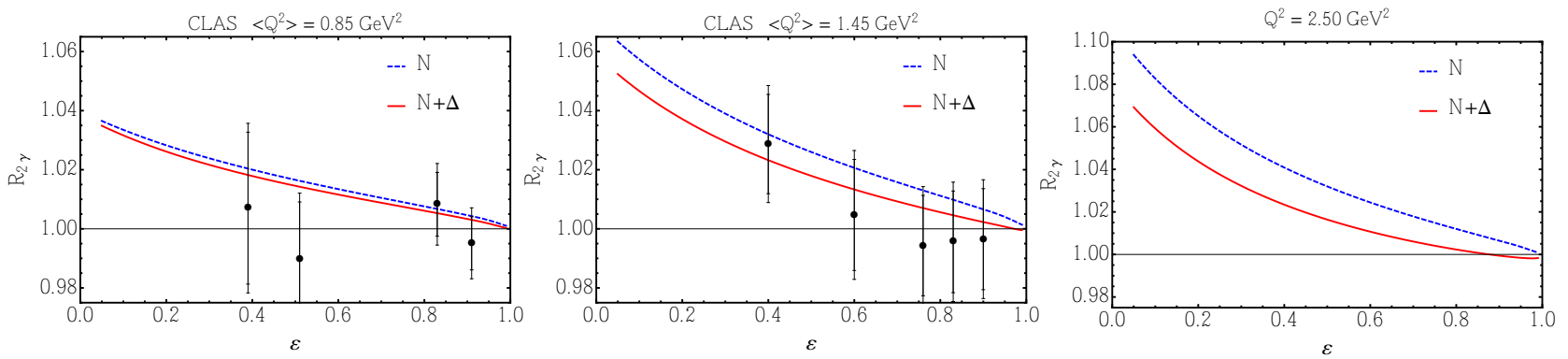

Figure 2.10: Ratio of $e^{+} p / e^{-} p$ elastic cross sections at various values of $Q^{2}$, after accounting for conventional radiative corrections. The data are from Ref. [20]. Figure taken from Ref. [54].

haeghen [59], transition form factors of arbitrary shape can be used. In particular, no reparametrization of the form factors in terms of a sum of monopoles is needed. Their calculation of the ratio of $e^{+} p$ to $e^{-} p$ cross sections over the range $Q^{2}=0.85-2.50 \mathrm{GeV}^{2}$ is shown in Fig. 2.10. The increasing importance of the $\Delta$ contribution as $Q^{2}$ increases is apparent from these plots.

The ratio of $e^{+} p$ to $e^{-} p$ cross sections, denoted by $R_{2 \gamma} \approx 1-2 \delta_{\gamma \gamma}$, is a direct measure of TPE effects. This is discussed more fully in Section 3. Gorchtein [51, 60] has recently made a modelindependent analysis of corrections to $R_{2 \gamma}$ in forward kinematics (forward angles, low $Q^{2}$ ) using the dispersive method. In forward kinematics the imaginary part of the TPE amplitude is related to the total photoabsorption cross section, a result first derived by Brown [61].

Tomalak and Vanderhaeghen [62] also considered the $e^{+} p / e^{-} p$ ratio at forward angles and small $Q^{2}$. The inelastic TPE contribution was expressed as a dispersion integral over the unpolarized proton structure functions $F_{1}^{\gamma}$ and $F_{2}^{\gamma}$. Their calculation goes beyond the known leading terms in the $Q^{2}$ expansion by keeping the full momentum-dependence of the proton structure functions. In the range of small $Q^{2}$, their result is in good agreement with the empirical TPE fits to existing data.

\subsubsection{QCD-based approaches at high $Q^{2}$}

The partonic approach for TPE calculations was developed in Refs. [47, 48]. It is based on applying a formalism of Generalized Parton Distributions (GPD) to the amplitude of wide-angle nucleon Compton scattering [63]. This approach assumes that a handbag mechanism is dominant (Fig. 2.11), with the electron-parton scattering proceeding on an individual parton. parametrizations of GPDs are obtained by fitting the global data on nucleon form factors [64].

Calculations of TPE using quark degrees of freedom for large transferred momenta include the QCD factorization approach [49,50], and parametrizations backed by Soft-Collinear Effective Theory (SCET) [65]. Borisyuk and Kobushkin [49] pointed out that within a pQCD framework, the TPE 

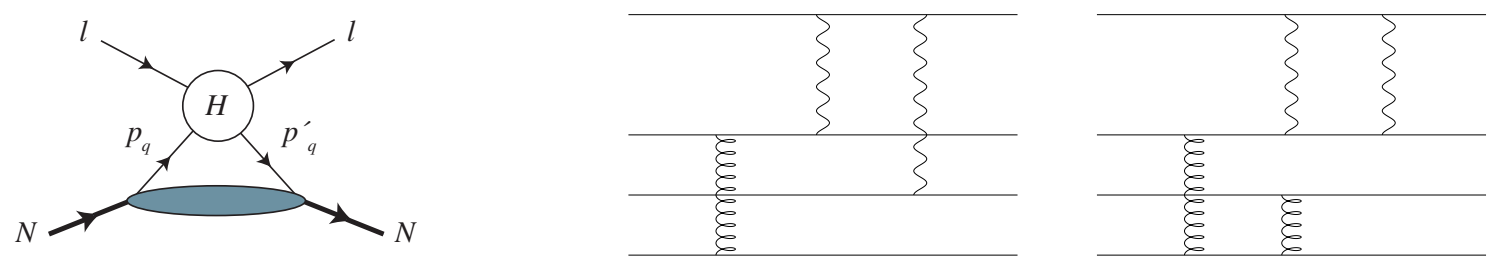

Figure 2.11: Left: TPE diagram in the GPD-based approach to $e N$ scattering at high $Q^{2}[47,48]$. Both photons interact with the same quark, while the others are spectators. Right: Sample TPE diagrams in the QCD factorization approach. For the leading order term the photons interact with different quarks, with a single gluon exchange. The interaction of two photons with the same quark is of subleading order in this approach, as it involves two gluons. Figures taken from Ref. [49].
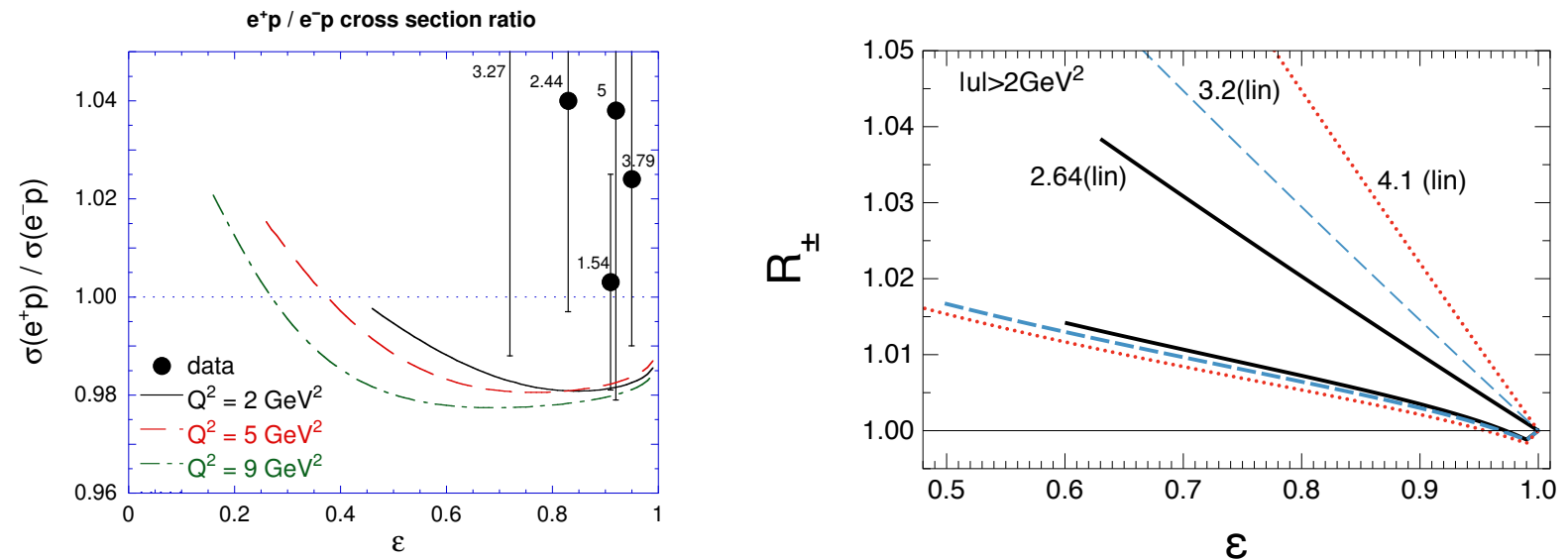

Figure 2.12: Left: Ratio of $e^{+} p / e^{-} p$ elastic cross sections, taken from Ref. [48]. The GPD calculations for the TPE correction are for three fixed $Q^{2}$ values of 2,5 , and $9 \mathrm{GeV}^{2}$, for the kinematical range where $-u$ is above $M^{2}$. Also shown are early SLAC data [66], with $Q^{2}$ above $1.5 \mathrm{GeV}^{2}$. The numbers near the data give $Q^{2}$ for that point in $\mathrm{GeV}^{2}$. Right: Ratio of $e^{+} p / e^{-} p$ at high $Q^{2}$ calculated in the QCD factorization approach [65]. Also shown for comparison are the results (labelled lin) from the from the phenomenological fits of Ref. [67]. Figure taken from Ref. [65].

diagram where the photons both interact with the same quark is subleading in comparison to the diagram where the photons interact with different quarks. As illustrated in Fig. 2.11, the second diagram requires two gluons, whereas the first diagram requires only one. Similar conclusions were reached by Kivel and Vanderhaeghen [50].

Kivel and Vanderhaeghen [65] calculated the TPE corrections in the QCD factorization approach formulated in the framework of soft-collinear effective theory. This technique allows them to develop a description for the soft-spectator scattering contribution, which is found to be important in the region of moderately large scales. Predictions of the GPD and QCD factorization models for the ratio of $e^{+} p / e^{-} p$ elastic cross sections at high $Q^{2}$ are shown in Fig. 2.12. Also shown for comparison are the predictions from a phenomenological fit to polarization and cross section data [67]. The predictions from phenomenological fits are considerable larger than the QCD factorization predictions.

\subsection{Two-photon exchange for spin polarization effects}

Two-photon exchange also has an effect on the polarization observables of electron-nucleon scattering. For double-polarization correlations, with both polarized electrons and a polarized recoil nucleon (or the nucleon target), TPE alters the dependence of the target asymmetry or recoil polarization, given in Born approximation by the expressions of Eqs. (2.11) and (2.12). This is caused by the additional spin dependence of the real part of the TPE amplitude beyond soft-photon contributions, Eqs. (2.20) and (2.21). The ratio of the recoil proton polarizations in the scattering plane can be expressed in terms of the real part of the generalized TPE form factors of Eq. $(2.27)[29,48]$. 


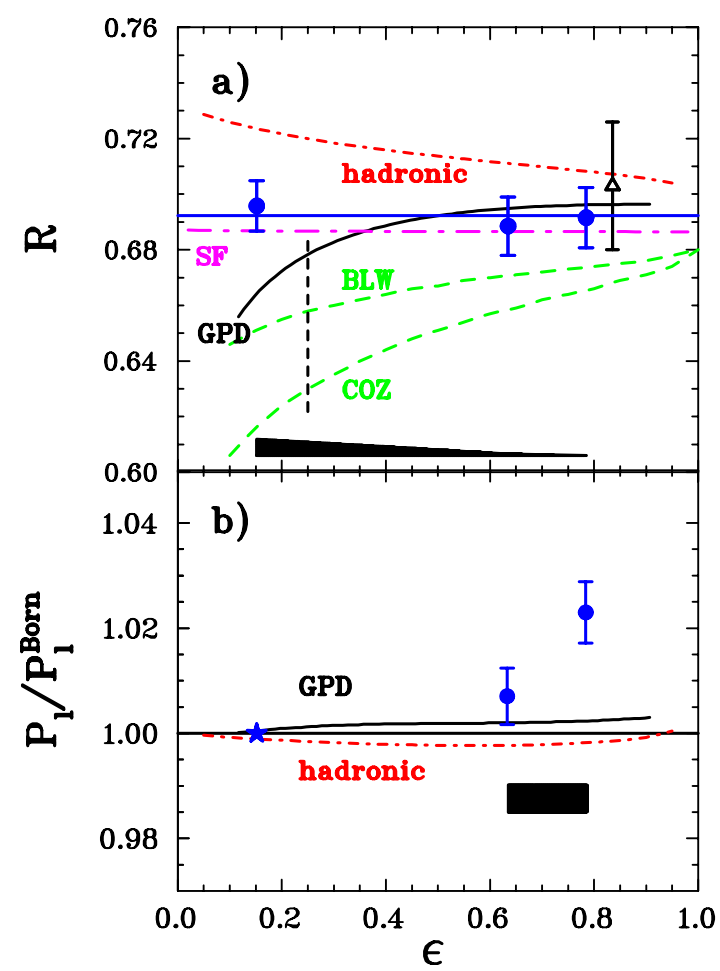

Figure 2.13: a) Experimental results for $R$ as a function of $\epsilon$ with statistical uncertainties, from Ref. [68]. The theoretical predictions are from: [69] (hadronic), [48] (GPD), [50] (COZ and BLW). b) $P_{\ell} / P_{\ell}^{B o r n}$ as a function of $\epsilon$. The pointto-point systematic uncertainties, shown with a band in both panels, are relative to the largest $\epsilon$ kinematic in a) and relative to the smallest $\epsilon$ kinematic in b). The star indicates the $\epsilon$ value at which the analyzing power is determined.

Experimental tests were conducted at Jefferson Lab [68], and the results are shown in Fig. 2.13. While the polarization ratio is consistent with the Born approximation within experimental errors, the longitudinal polarization transfer $P_{L}$ revealed a noticeable deviation at forward scattering angles.

In the plane-wave Born approximation, single-spin asymmetries (SSA) are zero due to time-reversal invariance and parity conservation in the electromagnetic interaction. But TPE is capable of generating SSA via the imaginary part of the amplitude. The corresponding spin-momentum correlation can be defined by a parity-even product $\boldsymbol{\xi} \cdot\left(\boldsymbol{k} \times \boldsymbol{k}^{\prime}\right)$, where $\boldsymbol{k}\left(\boldsymbol{k}^{\prime}\right)$ is the initial (final) electron momentum, and the vector $\boldsymbol{\xi}$ defines the polarization vector of either the electron or the nucleon. This form implies that the scattering asymmetry arises due to the polarization component oriented normal to the scattering plane.

A similar asymmetry in a pure QED process of electron-muon scattering due to two-photon exchange was calculated by Barut and Fronsdal [70]. Later calculations [71] for Moller scattering $e^{-}+$ $e^{-} \rightarrow e^{-}+e^{-}$also included radiative corrections to the asymmetry. SLAC experiment E158 confirmed the theoretical predictions within the statistical uncertainty [72]: $A_{n}(\exp )=7.04 \pm 0.25$ (stat) ppm versus $A_{n}$ (theory) $=6.91 \pm 0.04 \mathrm{ppm}$.

Early calculations of SSA in elastic ep-scattering were done by De Rujula et al. [73, 74] for the case of a transversely polarized proton target. They pointed out that a nonzero SSA is due to the absorptive part of the non-forward Compton amplitude for off-shell photons scattering from nucleons. Pasquini and Vanderhaeghen [75] modelled the virtual Compton amplitude by single-pion intermediate states, in the approach that is well justified by $S$-matrix unitarity for energies below the threshold of two-pion production. They made predictions for both beam and target SSA, and obtained good agreement with MAMI data [76].

In the formalism of Refs. [47, 48], the SSA, denoted as $A_{n}$, can be expressed in terms of the 


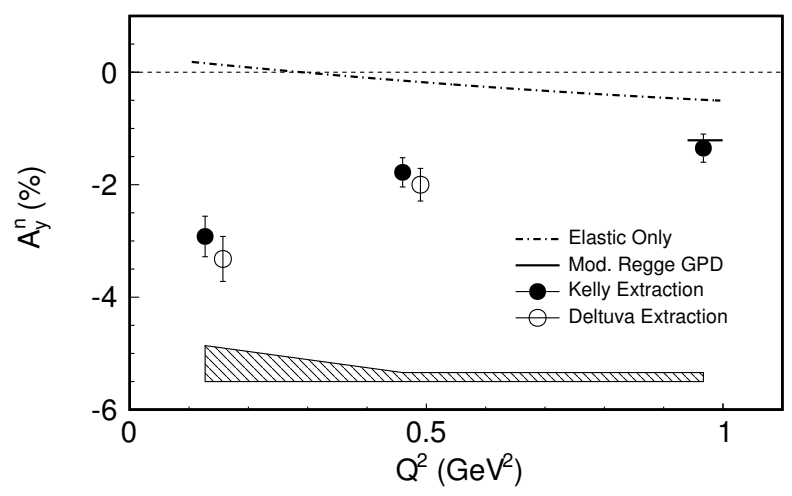

Figure 2.14: Results for the neutron asymmetries [77] $A_{\mathrm{n}}^{\mathrm{n}}$, as a function of $Q^{2}$. Uncertainties shown on the data points are statistical, while the systematic uncertainties are shown by the band at the bottom. The elastic contribution to the intermediate state is shown by the dot-dash line [78], and at $Q^{2}=0.97 \mathrm{GeV}^{2}$, the GPD calculation of Refs. [47, 48] is shown by the short solid line.

imaginary part of the generalized TPE form factors of Eq. (2.27) [29, 48]:

$$
\begin{aligned}
A_{n} & =\sqrt{2 \varepsilon(1+\varepsilon) \tau} \frac{1}{\sigma_{R}^{\text {meas }}}\left\{-G_{M} \operatorname{Im}\left(\tilde{F}_{1}-\tau \tilde{F}_{2}+\nu \tilde{F}_{3}\right)\right. \\
& \left.+G_{E} \operatorname{Im}\left(\tilde{F}_{1}+\tilde{F}_{2}+\left(\frac{2 \varepsilon}{1+\varepsilon}\right) \nu \tilde{F}_{3}\right)\right\} .
\end{aligned}
$$

The asymmetry $A_{n}$ was measured in Jefferson Lab experiment E05-015 [77] on a polarized ${ }^{3} \mathrm{He}$ target, designed to obtain the asymmetry on a single neutron. The results are compared with the theoretical predictions [47, 48] in Fig. 2.14 at $Q^{2} \approx 1 \mathrm{GeV}^{2}$, where the GPD approach is believed to be applicable.

If elastic scattering occurs on an unpolarized target, but the electron beam has a polarization component normal to the scattering plane, the corresponding asymmetry is also nonzero due to TPE. Transverse beam SSA for scattering in the Coulomb field of a nucleus was calculated a long time ago by Mott [79]. Examples of more recent work for the scattering on large- $Z$ nuclei may be found in [80, 81], where the Dirac equation is solved for the electron wave function in Coulomb field of nuclei. For low- $Z$ targets both TPE and Distorted-Wave Born Approximation calculations yield the same results for the beam SSA to order $\mathrm{O}(\alpha)$ that has the following behavior at small scattering angles $\theta_{e}<<1$ :

$$
A_{n}^{e} \propto \frac{\alpha m_{e} \theta_{e}^{3}}{E_{e}} .
$$

For electron scattering at $\mathrm{GeV}$ energies this formula predicts rather small asymmetries, around $10^{-10}$ for $\theta<10^{\circ}$ at Jefferson Lab conditions [80]. However, when we consider inelastic excitations of a nucleon, the predictions for the asymmetry are dramatically different. Theoretical calculations for a nucleon target [82-84] predicted that above the nucleon resonance region the beam SSA (a) does not decrease with beam energy, and (b) is enhanced by large logarithms due to exchange of hard collinear virtual photons. The expression for the asymmetry is the simplest in the diffractive regime and small scattering angles, where the virtual Compton amplitude can be related via the optical theorem to the total cross section of photoproduction on a nucleon by real photons, $\sigma_{\gamma p}$, that varies only slowly with energy. It allows for an exact calculation of the loop integral for the imaginary part of TPE amplitude, resulting in the following expression for the beam asymmetry in the high-energy diffractive regime:

$$
A_{n}^{e}=-\sigma_{\gamma p} \frac{m_{e} \sqrt{Q^{2}}}{8 \pi^{2}} \frac{G_{E}}{F_{1}^{2}+\tau F_{2}^{2}}\left(\log \left(\frac{Q^{2}}{m_{e}^{2}}\right)-2\right) \exp \left(-b Q^{2}\right),
$$




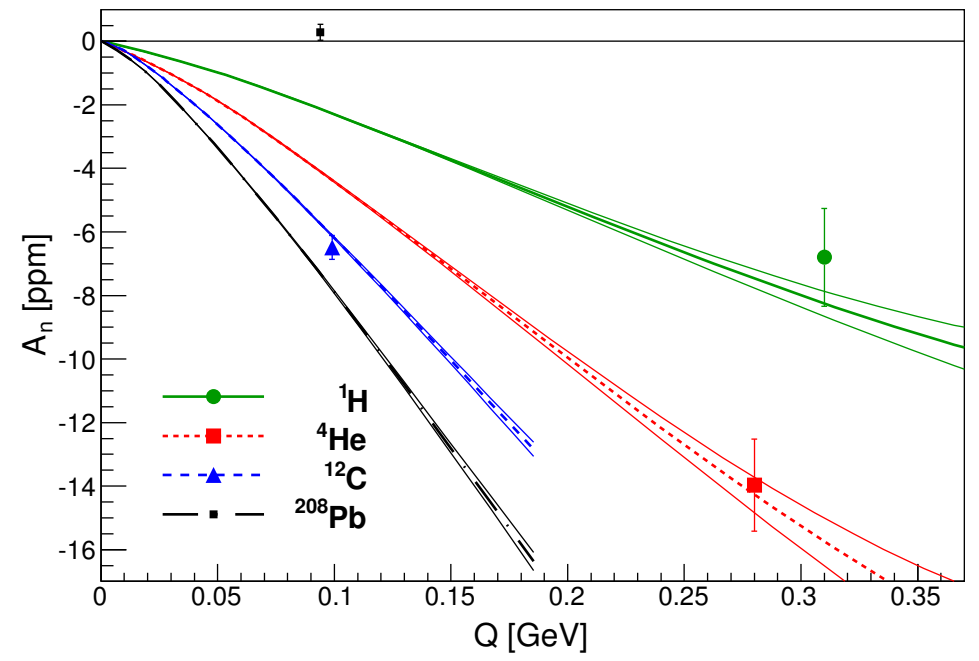

Figure 2.15: Extracted physics asymmetries $A_{n}$ versus $Q$ from Ref. [86]. Each curve, specific to a particular nucleus as indicated, is a theoretical calculation from Ref. [85].

where the parameter $b$ describes the slope of a non-forward Compton amplitude for the nucleon target, and $F_{1(2)}$ are Dirac (Pauli) form factors of a nucleon. If we compare the magnitude of the beam SSA predicted by Eqs. (2.29) and (2.30), one can see that for small angles and high energies the diffractive mechanism may exceed the Coulomb one by several orders of magnitude. We can also apply this approach in the nucleon resonance region, where the photoabsorption cross section, $\sigma_{\gamma p}$, strongly varies with photon energy. In this case the corresponding factor in the asymmetry is an integral [85] over the lab photon energy $\omega=\left(W^{2}-M^{2}\right) /(2 M)$, with $W$ being an invariant mass of the intermediate excited nucleon state:

$$
\operatorname{Im} A_{1}^{\text {inel }}=\frac{1}{4 \pi^{2}} \frac{M}{E_{e}} \int_{0}^{E_{e}} d \omega \omega \sigma_{\gamma N}(\omega) \log \left(\frac{Q^{2}}{m^{2}}\left(\frac{E_{e}}{\omega}-1\right)^{2}\right) .
$$

As long as the electron scattering angle is small, so that the nucleon Compton amplitude can be obtained by extrapolation from the forward limit, this unitarity-based approach gives a good description of experimental data that accompany the measurements on parity-violating electron scattering for a broad range of electron energies. Extension to the case of nuclei is straightforward, with the cross section replaced by the corresponding photonuclear cross section [85]. In particular, there is good agreement with data from HAPPEX and PREX experiments at Jefferson Lab [86] obtained on a proton and light nuclei, ${ }^{4} \mathrm{He}$ and ${ }^{12} \mathrm{C}$ Fig. 2.15. However, a significant disagreement with theory was observed for a high- $Z$ target ${ }^{208} \mathrm{~Pb}$ both in sign and magnitude: $A_{n}=0.28 \pm 0.25 \mathrm{ppm}$ (experiment) against $\approx-8 \mathrm{ppm}$ (theory). A possible reason for the disagreement is an effect of Coulomb distortion that grows linearly with a charge of a nucleus and may become significant for this case. A theoretical approach that combines Coulomb distortion and intermediate-state inelastic excitations is required for this case, while experiments with intermediate-mass nuclei, such as ${ }^{48} \mathrm{Ca}$ [87], could provide valuable information on transition between different dynamical mechanisms for the asymmetry generation. In the meantime, new preliminary results from the QWeak collaboration at Jefferson Lab [88] appear to be in good agreement with theory. In summary, SSA on the nucleon and nuclei provide valuable new information on the nucleon Compton amplitude and multi-photon effects in scattering on nuclei. 

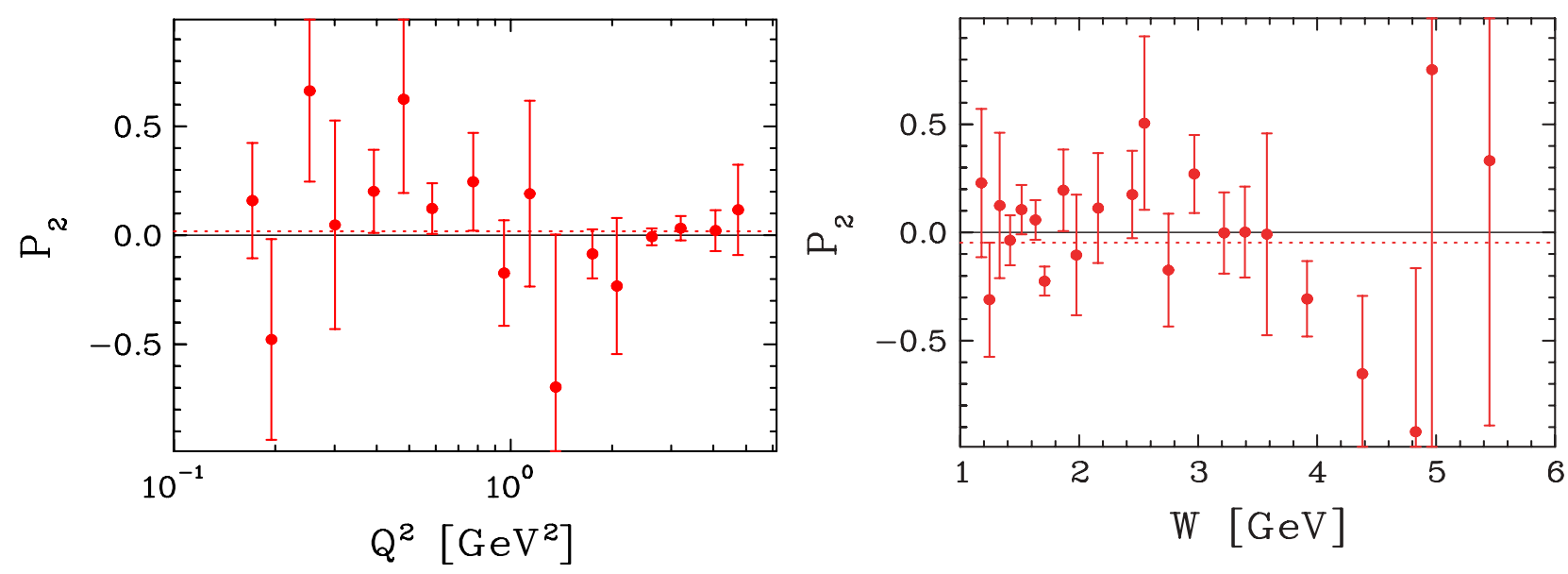

Figure 3.1: Values of $P_{2}$ for elastic (left) and inelastic data (right) as functions of $Q^{2}$ and $W$, respectively. The red dotted lines correspond to $\left\langle P_{2}\right\rangle=0.019 \pm 0.027$ for the elastic data and $-0.048 \pm 0.036$ for the inelastic data. Figure adapted from Ref. [89].

\section{Experimental observation of TPE}

\subsection{Nonlinearity of Rosenbluth data}

One technique employed as an indirect determination of TPE is to search for nonlinearity in the Rosenbluth data as a function of $\varepsilon$. In the OPE approximation, the reduced cross section $\sigma_{R}$ of Eq. (2.9) depends linearly on $\varepsilon$. From Fig. 2.3 it is clear that $\delta_{\gamma \gamma}$ itself varies at least linearly with $\varepsilon$. Therefore the measured reduced cross section $\sigma_{R}^{\text {meas }}$ of Eq. (2.23) will have a quadratic or higher order dependence on $\varepsilon$. Assuming a parametrization of the form $\delta_{\gamma \gamma}=A(1-\varepsilon)$, consistent with the condition that $\delta_{\gamma \gamma} \rightarrow 0$ as $\varepsilon \rightarrow 1$, one finds

$$
\sigma_{R}^{\text {meas }} \approx(1+A) \tau G_{M}^{2}+\left[(1+A) G_{E}^{2}-\tau G_{M}^{2}\right] \varepsilon-A G_{E}^{2} \varepsilon^{2} .
$$

A similar quadratic dependence would appear in elastic electron-nucleus or inelastic electron scattering data used in $\sigma_{L} / \sigma_{T}$ separations.

At low values of $Q^{2}$, where $G_{E}$ dominates, any indication of a quadratic behavior of $\sigma_{R}^{\text {meas }}$ would be strong empirical evidence for TPE effects, as seen by the last term in Eq. (3.1). An analysis of both elastic and inelastic electron scattering data was performed by Tvaskis et al. [89] in which they fit the reduced cross section with a parametrization

$$
\sigma_{R}=P_{0}\left[1+P_{1}(\varepsilon-0.5)+P_{2}(\varepsilon-0.5)^{2}\right]
$$

The form was chosen so that the nonlinear term, $P_{2}$, is a fractional contribution relative to the average cross section. It does not require $\delta_{\gamma \gamma} \rightarrow 0$ as $\varepsilon \rightarrow 1$. The resulting values of $P_{2}$ are shown in Fig. 3.1. The average values, $\left\langle P_{2}\right\rangle=0.019 \pm 0.027$ for elastic data and $-0.048 \pm 0.036$ for the inelastic data, are both consistent with no nonlinear effects. One also sees that the vast majority of the points in both plots lack the sensitivity to discern nonlinear effects. The three most precise values of $P_{2}$ come from the Jefferson Lab "Super Rosenbluth" experiment [11]. Individually, these points are entirely consistent with $P_{2}=0$. A calculation of $P_{2}[47,48]$ using partonic calculations $[47,90]$ yielded values consistent with what was observed in Ref. [89]. Similar analyses on ep [91] and ed [92] elastic scattering found similar negligibly small nonlinearities.

Equation (3.1) reveals the challenge of finding evidence for TPE effects. The nonlinear term requires one to look at low $Q^{2}$ where $G_{E}$ is largest, but this is precisely where one expects TPE effects to be small. At higher values of $Q^{2}$, where the $G_{E}$ term is suppressed, Eq. (3.1) suggests the $\varepsilon$ dependence 


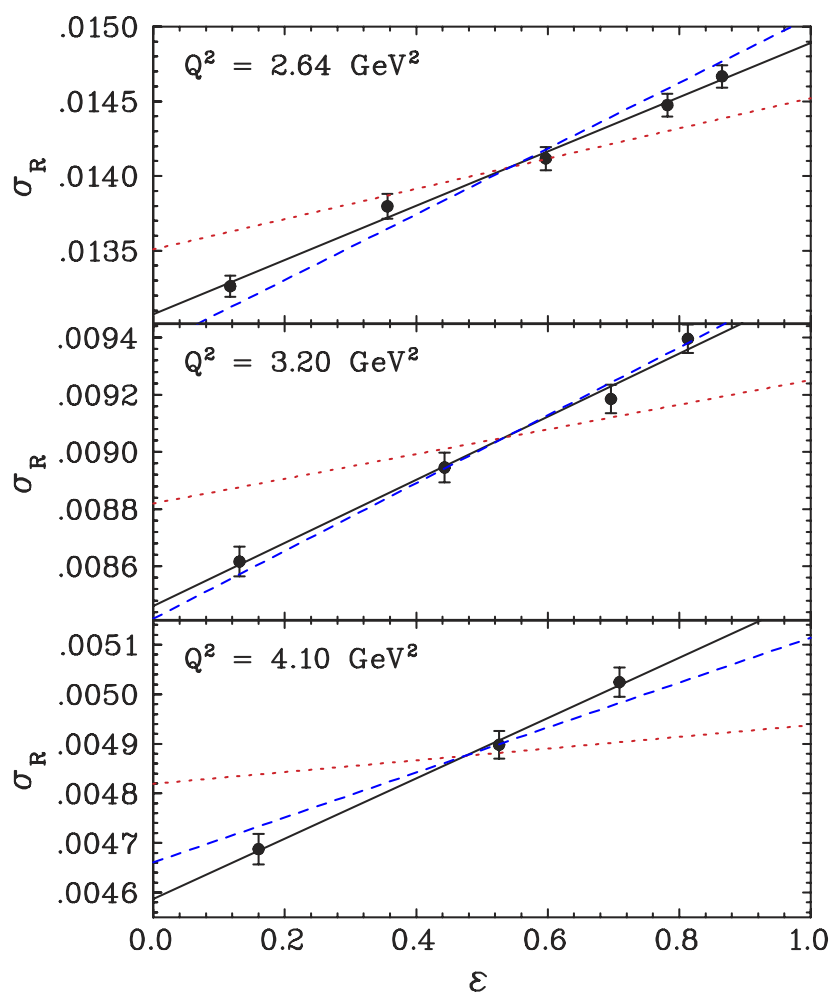

Figure 3.2: Reduced cross sections with linear fit (solid line) used to extract the proton form factors. The dotted line indicates the slope predicted by the polarization transfer experiments and the dashed line shows the slope expected from scaling $\left(\mu_{p} G_{E} / G_{M}\right)$. Figure adapted from Ref. [11].

would come mostly from the $G_{M}$ part of the second term. However, a Rosenbluth separation by itself would not reveal any TPE effect, but rather only lead to an incorrect extraction of the form factors. Since one expects a positive slope for $\delta_{\gamma \gamma}$ as a function of $\varepsilon$, i.e. $B>0$, this would lead to an increase in the $\varepsilon$-dependent slope in the of the measured reduced cross section. Figure 3.2 shows the Rosenbluth separation results from Ref. [11] that also includes lines with slopes inferred from polarization recoil data, which are, indeed, less than the fitted slopes.

\subsection{Direct measurements of TPE corrections to $\sigma_{R}$}

One way to directly determine the size of the TPE effect is to measure the ratio of $e^{-} p$ and $e^{+} p$ elastic scattering cross sections [93, 94]. The TPE correction, $\delta_{\gamma \gamma}$, arising from the interference between one- and two-photon exchange amplitudes, has opposite signs for electrons and positrons, whereas most of the other radiative corrections cancel to first order in the ratio. (From Eq. (2.19), $\mathcal{M}_{\gamma}$ changes sign for positrons, whereas $\mathcal{M}_{\gamma \gamma}$ does not.) The largest of the corrections that don't cancel in the ratio is the interference between lepton and proton bremsstrahlung radiation, $\delta_{\mathrm{b}, e p}$, which is of comparable size to $\delta_{\gamma \gamma}$. Taken together, $\delta_{\text {odd }} \equiv \delta_{\gamma \gamma}+\delta_{\mathrm{b}, e p}$ constitute the charge-odd radiative corrections.

The charge-even radiative corrections, denoted $\delta_{\text {even }}$, include logarithmically enhanced terms $\sim$ $\log \left(Q^{2} / m_{e}^{2}\right)$ arising from electron bremsstrahlung, vacuum polarization, and vertex corrections, and are therefore not small. No such enhancements occur for $\delta_{\text {odd }}$, so that typically $\left|\delta_{\text {odd }}\right| \ll\left|\delta_{\text {even }}\right|$.

As detailed in Refs. [20, 95], the ratio of the measured $e^{ \pm} p$ elastic scattering cross sections can therefore be written as

$$
R_{\text {meas }}^{ \pm}=\frac{\sigma\left(e^{+} p\right)}{\sigma\left(e^{-} p\right)} \approx \frac{1+\delta_{\text {even }}-\delta_{\text {odd }}}{1+\delta_{\text {even }}+\delta_{\text {odd }}} \approx 1-\frac{2 \delta_{\text {odd }}}{1+\delta_{\text {even }}}
$$


After correcting the experimental ratio $R_{\text {meas }}^{ \pm}$for the calculated $\delta_{\mathrm{b}, e p}$ and $\delta_{\text {even }}$ corrections, $\delta_{\gamma \gamma}$ can be extracted from the ratio

$$
R_{2 \gamma} \approx 1-2 \delta_{\gamma \gamma}
$$

There have been several attempts to measure TPE corrections from the ratio of positron scattering to electron scattering cross sections. These include several experiments in the 1960's and 1970's [66, 96103]. These experiments showed no significant TPE effect, and will be discussed in Section 3.3. With the rise of the Rosenbluth polarization transfer discrepancy there was a renewed effort at a direct measurement of the TPE effect. Three recent experiments have released results:

- The VEPP-3 [104, 105] experiment used monoenergetic beams from a storage ring incident on an internal gas target. The VEPP-3 experiment used beams of 1.0 and $1.6 \mathrm{GeV}$ with non-magnetic spectrometers at angles from $15^{\circ}$ to $105^{\circ}$ and is discussed in Section 3.5.

- The CLAS TPE experiment [20, 106] ran at the Thomas Jefferson National Accelerator Facility (Jefferson Lab) and used a mixed beam of positrons and electrons with beam energies from 0.8 to $3.3 \mathrm{GeV}$ allowing for simultaneous detection of electron and positron scattering events in the CEBAF Large Acceptance Spectrometer (CLAS), while also covering a wide range in $\varepsilon$ and $Q^{2}$. This experiment will be discussed in Section 3.6.

- The OLYMPUS [107], like the VEPP-3 experiment, used a monoenergetic beam from a storage ring incident on an internal gas target. The beam energy was $2.01 \mathrm{GeV}$ and the scattered particles were detected with the MIT Bates BLAST detector and covered a continuous range of scattering angles from $25^{\circ}$ to $75^{\circ}$. OLYMPUS is discussed in Section 3.7.

\subsection{Early measurements of TPE}

Although even early on the size of the TPE effect was expected to be small, there were several attempts during the 1960s and 1970s to do a direct measurement of it by measuring the ratio $R_{2 \gamma}$. The earliest of these was Yount et al. [96] in 1962 using the Stanford Mark III electron accelerator. The electrons were either sent directly to the target or used to produce a positron beam. The leptons passed through a reversible-field momentum-analyzing magnet on their way to a liquid hydrogen target with beam currents determined by a Faraday cup and, independently, by ion chambers in the beamline. Scattered leptons were detected in a magnetic spectrometer. Four data points were measured, one at $205 \mathrm{MeV}$ and a scattering angle of $30^{\circ}$ and three at $307 \mathrm{MeV}$ and scattering angles of $30^{\circ}, 45^{\circ}$, and $130^{\circ}$. All four data points were consistent with $R_{2 \gamma}=1$.

Following Yount et al. [96], a series of eight more electron-positron experiments were conducted over the next 13 years using much the same technique. These included experiments at the Stanford Mark III accelerator [97], the Cornell Synchrotron [98, 100, 101], DESY [99, 103], the Orsay Linear Accelerator [102], and SLAC [66]. The results of these experiments are shown in Fig. 3.3 as a function of $Q^{2}$ though the data vary in their value of $\varepsilon$. It is clear from this plot that these measurements are statistically consistent with $R_{2 \gamma}=1$. If, as expected, the TPE effect is only a few percent, the uncertainties of these results are simply too large to discern the effect. It must be said, that these measurements are extremely difficult, even with today's technology, and maintaining precision control of systematic uncertainties is daunting as is obtaining the statistical accuracy needed for a few percent measurement.

Soon after it became apparent that there was a discrepancy between Rosenbluth and polarizationtransfer measurement of the proton form factors, these data were reexamined [93]. While no discernible $Q^{2}$ dependence was found, a linear fit as a function of $\varepsilon$ for $Q^{2}<2 \mathrm{GeV}^{2}$ resulted in a $5.7 \%$ increase of $R_{2 \gamma}$ with decreasing $\varepsilon$. Though the uncertainty on the fit was quoted as $\pm 1.8 \%$, the fit is largely driven by just few low $\varepsilon$ data points, most of which have $Q^{2}<0.5$, which is well below where there is any significant discrepancy in the form factor data. Given the lack of detail in most of the papers from which these data came, specifically regarding the radiative corrections, it is unclear if the data 


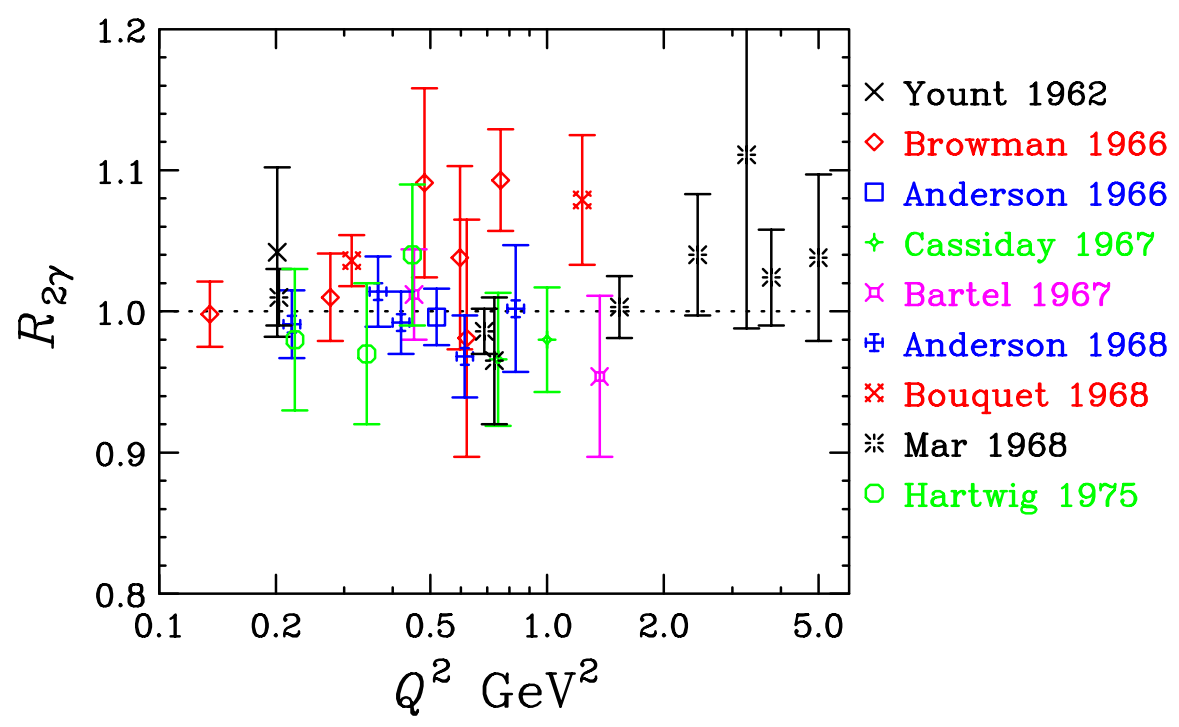

Figure 3.3: $R_{2 \gamma}$ data from the 1960's and 70's. Data are plotted with a logarithmic scale in $Q^{2}$. The key for the points are shown for Yount 1966 [96], Browman 1966 [97], Anderson 1966 [98], Cassiday 1967 [100], Bartel 1967 [99], Anderson 1968 [101], Bouquet 1968 [102], Mar 1968 [66], and Hartwig 1975 [103].

have been consistently corrected. A plot of these data as a function of $\varepsilon$ are shown in Fig. 3.4 along with a reproduction of the fit from Ref. [93].

\subsection{TPE in experiments with spin}

As discussed in section 2.3, TPE can have a significant effect on the Rosenbluth or longitudinaltransverse (LT) separation technique's determination of $\mu_{p} G_{E} / G_{M}$ while having a lesser effect on the results obtained using polarization transfer techniques (PT). Nevertheless, as outlined in section 2.4, TPE must also be taken into account in spin-dependent electron-nucleon scattering as well as elsewhere. A number of experiments measuring polarization observables have investigated the role of TPE. Some of these experiments will be briefly reviewed in this section.

The GEp2 $\gamma$ Collaboration [68] investigated effects beyond one-photon exchange (OPE) or the Born approximation by measuring polarization transfer in the reaction $\mathrm{H}\left(\vec{e}, e^{\prime} \vec{p}\right)$. The experiment ran in Hall $\mathrm{C}$ at Jefferson Lab using a longitudinally polarized electron beam with $82 \%-86 \%$ polarization incident on a $20 \mathrm{~cm}$ long liquid hydrogen target. Scattered electrons were detected in the BigCal lead glass calorimeter. The coincident protons were detected in the High Momentum Spectrometer and two, $55 \mathrm{~cm}$ thick, $\mathrm{CH}_{2}$ analyzer blocks in the spectrometer's focal plane determined the proton polarization. Three electron beam energies were used: $1.87,2.84$, and $3.63 \mathrm{GeV}$ and the detector angles were adjusted to obtain results at a common $Q^{2}=2.49 \mathrm{GeV}^{2}$. The data were analyzed to determine the proton form factor ratio, $\mu_{p} G_{E} / G_{M}$, that sparked the current interest in TPE. The results have have been shown already in Fig. 2.13.

The ratio is consistent with the Born approximation over the measured range $0.152<\varepsilon<0.785$ at the $1.5 \%$ level suggesting little or no effect at this $Q^{2}$ or possibly that TPE effects cancel in the ratio. They also measured the longitudinal polarization transfer $P_{L}$, which showed a significant $(\sim 2 \%)$ increase with $\varepsilon$ compared to the Born expectation. This implies a similar, cancelling behavior for $P_{T}$ to explain the result for $R$ and that TPE may not be negligible in polarization transfer observables at forward angles.

The SAMPLE experiment [108] ran at MIT-Bates. The experiment employed polarized, $200 \mathrm{MeV}$ electrons incident on a $40 \mathrm{~cm}$ long liquid hydrogen target. The polarization was $\sim 36 \%$. The electrons, scattered at back angles $130^{\circ}<\theta_{e}<170^{\circ}$, were detected in a large acceptance $(\sim 1.5 \mathrm{sr})$ air 


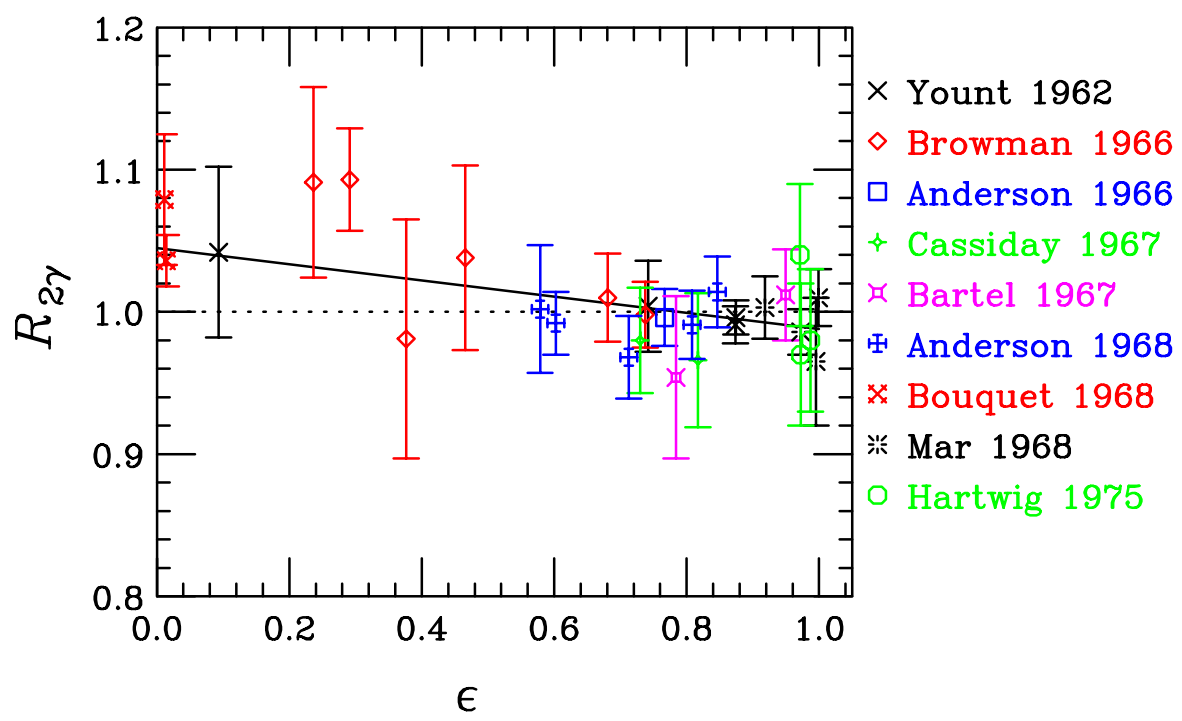

Figure 3.4: $R_{2 \gamma}$ data with $Q^{2}<2.0 \mathrm{GeV}^{2}$ from the 1960 's and 70 's. The key for the points are the same as in Fig. 3.3 . The line is a reproduction of the fit from Ref. [93].

Čerenkov detector. Initially, SAMPLE ran with longitudinally polarized electrons and measured the parity violating asymmetry, $A_{P V}$, to constrain the strange weak magnetic form factor [109]. Later, transversely polarized electrons were employed. Single spin asymmetries (SSA) as already pointed out in section 2.4, vanish in the Born approximation because of time-reversal invariance and parity conservation in purely electromagnetic interactions. However, TPE can produce SSA when either the beam or target is transversely polarized. The SAMPLE result was $A_{n}=-15.4 \pm 5.4 \mathrm{ppm}$ (parts per million) at the average scattering angle of $146.1^{\circ}$ corresponding to $Q^{2}=0.1 \mathrm{GeV}^{2}$. The result is almost two standard deviations below the expected asymmetry from a calculation that assumed only a proton propagator for the intermediate proton state in TPE.

Similarly, the A4 experiment [76] at the MAMI accelerator in Mainz measured $A_{n}$ at a $\operatorname{similar} Q^{2}$ but with higher electron beam energies of 569.3 and $855.2 \mathrm{MeV}$. A $10 \mathrm{~cm}$ liquid hydrogen target was used and an azimuthally symmetric array of $\mathrm{PbF}_{2}$ crystals covered the angular range $30^{\circ}<\theta_{e}<40^{\circ}$. The average squared momentum transfers were 0.106 and $0.230 \mathrm{GeV}^{2}$. The A4 results are:

$$
\begin{aligned}
& A_{n}\left(Q^{2}=0.106 \mathrm{GeV}^{2}\right)=-8.59 \pm 0.89(\text { stat }) \pm 0.75(\text { syst })(\mathrm{ppm}), \\
& A_{n}\left(Q^{2}=0.230 \mathrm{GeV}^{2}\right)=-8.52 \pm 2.31(\text { stat }) \pm 0.87 \text { (syst) }(\mathrm{ppm}) .
\end{aligned}
$$

Comparison with theoretical calculations at the time showed no agreement for the simple proton intermediate state though calculations including $\pi N$ intermediate states were much closer but still needed some tuning.

The E158 experiment [72] at SLAC was designed to make a precise measurement of the weak mixing angle, $\sin \theta_{W}{ }^{2}$, in Møller scattering. The experiment scattered longitudinally polarized electrons at $50 \mathrm{GeV}$ from a liquid hydrogen target and detected them $60 \mathrm{~m}$ downstream in an azimuthally symmetric ring of calorimeters that were segmented both radially and azimuthally. The parity violating asymmetry, $A_{P V}$, observed by reversing the helicity of the incident electron beam was measured to determine the weak mixing angle. However, in addition to measuring the longitudinal asymmetry, E158 made a series of runs with transverse electron polarization. The SSA, $A_{n}$, was measured at E158 for both Møller and ep scattering and the results reported for Run 2 in a Technical note [110]. The 
results are in good agreement with theoretical calculations by Dixon and Schreiber [71]:

$$
\begin{aligned}
A_{n}^{e e}(\operatorname{expt}) & =-7.04 \pm 0.25(\text { stat }) \pm 0.37(\text { syst })(\mathrm{ppm}) \\
A_{n}^{e e}(\text { theory }) & =-6.91 \pm 0.04(\mathrm{ppm}) \\
A_{n}^{e p}(\mathrm{expt}) & =+2.89 \pm 0.36(\text { stat }) \pm 0.17(\text { syst })(\mathrm{ppm}) \\
A_{n}^{e p}(\text { theory }) & =+3.16 \pm 0.32(\mathrm{ppm})
\end{aligned}
$$

Similarly, the G0 experiment $[111,112]$ was designed to measure the parity violating asymmetry, $A_{P V}$ in $e p$ elastic scattering and in quasi-elastic scattering $e d$. The experiment ran in Hall $\mathrm{C}$ at Jefferson Lab. Longitudinally polarized electrons at $3.031 \mathrm{GeV}$ were directed onto a $20 \mathrm{~cm}$ long liquid hydrogen target. A large, super-conducting, eight sector, toroidal spectrometer was used to momentum-analyze the reactions. This spectrometer was utilized in two modes: a forward angle mode detecting the recoil protons and a backward angle mode detecting the scattered electrons. By combining information from the electromagnetic nucleon form factors with form factors for the neutral weak currents the contributions of the lightest quark flavors can be extracted.

However, the G0 experiment also ran with transverse electron polarization and extracted information on TPE in forward-angle mode [113] and also in backward-angle mode [114]. With the spectrometer in forward angle mode, measurements were made at $Q^{2}=0.15$ and $0.25 \mathrm{GeV}^{2}$ detecting the recoil proton, and found the SSAs

$$
\begin{aligned}
& A_{n}^{e p}\left(Q^{2}=0.15 \mathrm{GeV}^{2}\right)=-4.06 \pm 0.99(\mathrm{stat})(\mathrm{ppm}) \\
& A_{n}^{e p}\left(Q^{2}=0.25 \mathrm{GeV}^{2}\right)=-4.82 \pm 1.87(\text { stat })(\mathrm{ppm})
\end{aligned}
$$

Again, calculations using simply the proton intermediate state did not approach the measured results while calculations including the $\pi N$ intermediate state were better but still not in agreement. Another calculation based on the optical theorem and total photo-production cross section over-shot the data. Running in backward angle mode, detecting the scattered electron in $e p$ elastic scattering, yielded

$$
\begin{aligned}
& A_{n}^{e p}\left(Q^{2}=0.362 \mathrm{GeV}^{2}\right)=-176.5 \pm 9.4(\mathrm{stat})(\mathrm{ppm}) \\
& A_{n}^{e p}\left(Q^{2}=0.687 \mathrm{GeV}^{2}\right)=-21.0 \pm 24.0(\text { stat })(\mathrm{ppm})
\end{aligned}
$$

These results, together with results from SAMPLE and A4, were in good agreement with the theoretical calculations of Pasquini and Vanderhaeghen [75] that interpreted the imaginary part of TPE in terms of doubly virtual Compton scattering using a phenomenological analysis of pion electroproduction observables.

The G0 Collaboration also measured $A_{n}$ for ed scattering:

$$
\begin{aligned}
& A_{n}^{e d}\left(Q^{2}=0.362 \mathrm{GeV}^{2}\right)=-108.6 \pm 7.2(\mathrm{stat})(\mathrm{ppm}), \\
& A_{n}^{\text {ed }}\left(Q^{2}=0.687 \mathrm{GeV}^{2}\right)=-55.7 \pm 78.0(\text { stat })(\mathrm{ppm}) .
\end{aligned}
$$

The deuteron SSA measurements can be unfolded to estimate the contributions from the proton, $A_{n}^{p}$, and neutron, $A_{n}^{n}$, separately:

$$
\begin{aligned}
& A_{n}^{p}\left(Q^{2}=0.362 \mathrm{GeV}^{2}\right)=-176.5 \pm 49.4(\text { stat })(\mathrm{ppm}) \\
& A_{n}^{p}\left(Q^{2}=0.687 \mathrm{GeV}^{2}\right)=-21.0 \pm 24.0(\text { stat })(\mathrm{ppm}) \\
& A_{n}^{n}\left(Q^{2}=0.362 \mathrm{GeV}^{2}\right)=+86.6 \pm 41.0(\text { stat })(\mathrm{ppm}) \\
& A_{n}^{n}\left(Q^{2}=0.687 \mathrm{GeV}^{2}\right)=-138.0 \pm 268.0(\text { stat })(\mathrm{ppm}) .
\end{aligned}
$$

The HAPPEX and PREX collaborations [86] measured the transverse beam asymmetry for elastic electron scattering from a number of different targets: $\mathrm{H},{ }^{4} \mathrm{He},{ }^{12} \mathrm{C}$, and ${ }^{208} \mathrm{~Pb}$. The results have already been shown (Fig. 2.15) and discussed. 

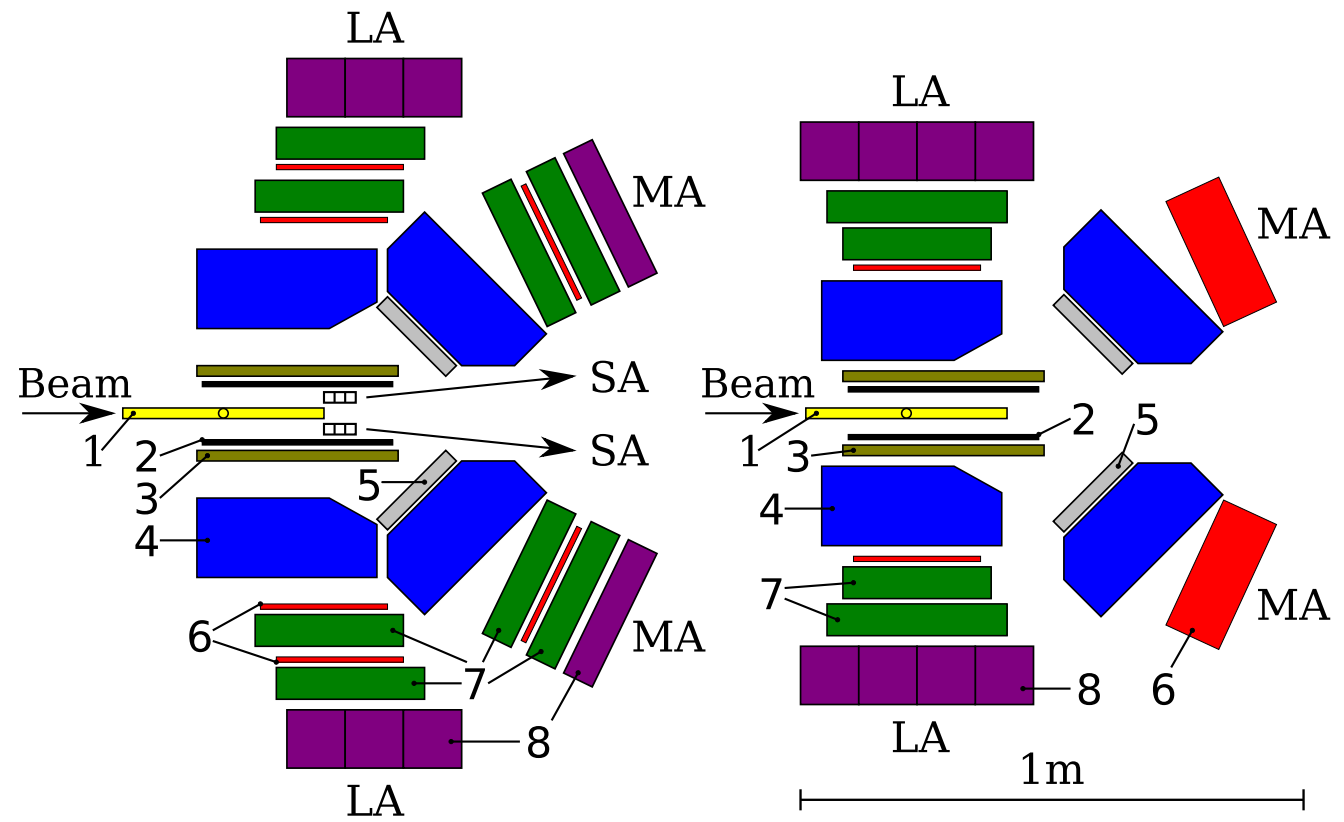

Figure 3.5: VEPP-3 TPE experiment detector configurations for the $1.6 \mathrm{GeV}$ run (left) and the $1.0 \mathrm{GeV}$ run (right). The labels refer to 1-target cell,; 2-beryllium sheet; 3-multiwire proportional chamber; 4-drift chamber; 5-acrylic glass; 6-plastic scintillator; 7-CsI crystals; 8-NaI crystals. LA corresponds to the large angle spectrometer, MA to the medium angle spectrometer, and SA to the small angle calorimeter. Figure adapted from Ref. [105].

The QWeak Collaboration [115] is also measuring $A_{n}$ for a number of different targets. For $e p$ elastic scattering at $1.155 \mathrm{GeV}$ and $Q^{2}=0.025 \mathrm{GeV}^{2}$, QWeak [88] found

$$
A_{n}^{p}\left(Q^{2}=0.025 \mathrm{GeV}^{2}\right)=-5.350 \pm 0.07 \text { (stat) } \pm 0.15(\mathrm{syst})(\mathrm{ppm}) .
$$

In summary, the single spin asymmetry measurements, while small, potentially can constrain the imaginary parts of the generalized form factors in a spin-dependent calculation of two-photon exchange. It is also clear that theoretical calculations must include resonance states in the intermediate propagator.

\subsection{The VEPP-3 experiment}

The first of the new era of direct TPE experiments to take data was done using the VEPP-3 storage ring in Novosibirisk, Russia [105, 116]. It used an internal hydrogen gas target and took data with beam energies of 1.6 and $1.0 \mathrm{GeV}$ during two separate running periods. The experiment used non-magnetic spectrometers (shown in Fig. 3.5), which guarantees identical acceptances for $e^{+} p$ and $e^{-} p$ events. This constituted a relative advantage for the VEPP-3 experiment compared to the CLAS and OLYMPUS experiments, which both used magnetic spectrometers and can lead to acceptance differences for the two types of events. There were two pairs of left-right symmetric spectrometers large angle (LA) spectrometers and medium angle (MA) spectrometers - along with a pair of left-right symmetric small angle (SA) calorimeters. The LA and MA spectrometers were placed at different angles for the two runs resulting in average lepton angles of $\left\langle\theta_{e}\right\rangle=66.2^{\circ}$ and $20.8^{\circ}$ for the $1.6 \mathrm{GeV}$ run and $75.4^{\circ}$ and $21.4^{\circ}$ for the $1.0 \mathrm{GeV}$ run, and were used to detect both leptons and protons. The components of each of the spectrometers is described in the caption of Fig. 3.5. Scattering angles were primarily determined by the multiwire proportional chambers and the drift chambers, while the other components were used to measure particle energy and to determine particle identification.

The experiment alternated between running with positron and electron beams but did not determine an absolute positron/electron normalizations. Instead, luminosity normalization points were take at 
small angles where hard TPE effects are expected to be small $[2,117]$ and $R_{2 \gamma}=1$. The SA arms detected only scattered leptons and were used only during the $1.6 \mathrm{GeV}$ run to determine the luminosity normalization, while MA events were used for luminosity normalization during the $1.0 \mathrm{GeV}$ run. For the $1.6 \mathrm{GeV}$ run a total of luminosity of $320 \mathrm{pb}^{-1}$ was recorded, while for the $1.0 \mathrm{GeV}$ run the luminosity was $600 \mathrm{pb}^{-1}$.

Elastic events were determined by a co-planarity cut and a series of cuts on the scattering angles and energies of the coincident lepton-proton pair. Remaining background was removed by simulations that included the most likely background events: $e p \rightarrow e^{\prime} n \pi^{+}, e p \rightarrow e^{\prime} p \pi^{0}, \gamma^{*} p \rightarrow n \pi^{+}$, and $\gamma^{*} p \rightarrow p \pi^{+} \pi^{-}$. The background was found to be less than $4 \%$ in the LA angle range and negligible for the other angle ranges.

The data were corrected for first-order radiative effects using a simulation with an event generator [118] that used a dipole parametrization of the for the proton form factors, a QED calculation beyond the soft-photon approximation for first-order bremsstrahlung, a vacuum polarization correction that included the hadronic contribution, and the soft TPE terms according to Mo and Tsai [7]. The generated events were fed into a simulation of the experiment and was run for both positrons and electrons, with radiative effects turned on and also assuming the exchange of a single virtual photon. The resulting ratios, $N_{\text {sim }}^{+} / N_{\text {sim }}^{0}$ and $N_{\text {sim }}^{-} / N_{\text {sim }}^{0}$ were then applied to the measured ratio of $R$ to give

$$
R_{2 \gamma}=R_{\text {meas }}^{ \pm} \frac{N_{\text {sim }}^{-} / N_{\text {sim }}^{0}}{N_{\text {sim }}^{+} / N_{\text {sim }}^{0}} .
$$

The total systematic uncertainty was very low for this experiment and varied between $0.08 \%$ to $0.32 \%$. The sources of systematic uncertainties include beam effects, kinematic cuts, background subtraction, and radiative corrections. For three of the four VEPP-3 data points the statistical uncertainty is a factor of 3-4 times larger than the systematic, while at the other data point the systematic uncertainty is about twice as large as the statistical uncertainty.

The results of the VEPP-3 experiment are shown in Fig. 3.6 along with older data at similar kinematics [97-99, 101] and various predictions [31, 35, 52, 119-121]. The results are in agreement with, but significantly more precise, than the older data.

It is important to note that the results are normalized to the luminosity normalization points taken at small scattering angles and assumed to have $R_{2 \gamma}=1$. So a direct comparison inferred from the plot does not does not reveal how well the data and predictions match. In principle, the data should be shifted so that the normalization point is at the value of $R_{2 \gamma}$ of the curve to which the data are being compared. The authors of Ref. [105] performed this exercise and found reasonable agreement with predictions of $[31,35,52]$ with $\chi_{\nu}^{2}$ from 1.0 to 4.19 , while predictions of [119-121] had $\chi_{\nu}^{2}>5$. The "no hard TPE effect" had $\chi_{\nu}^{2}=7.97$, which corresponds to ruling out the "no TPE" hypothesis at better than a $99 \%$ confidence level.

\subsection{The CLAS experiment}

The CLAS TPE experiment $[20,106]$ ran at the Thomas Jefferson National Accelerator Facility (Jefferson Lab). It had large coverage of kinematics with $0.85 \leq Q^{2} \leq 1.45 \mathrm{GeV}^{2}$ and nearly the entire interval of $\varepsilon$ from 0.2 to 0.9 . The experiment utilized a mixed beam of electrons and positrons with an effective beam energy of between 0.8 and $3.5 \mathrm{GeV}$. This mixed beam was produced by first bombarding a gold radiator (see Fig. 3.7) with a 110-140-nA, $5.6 \mathrm{GeV}$ electron beam. The electrons were diverted by the CLAS tagger magnet into a beam dump while the collimated photon beam struck a gold converter to produce electron-positron pairs. The leptons then passed through a three-dipole chicane, the first of which separated the leptons horizontally from the photon beam, which was prevented from reaching the target by a tungsten block within the chicane. The second and third dipoles recombined the lepton beams that were collimated en route to a liquid hydrogen target. The scattered leptons and the protons were detected in the CEBAF Large Acceptance Spectrometer (CLAS) [122]. 

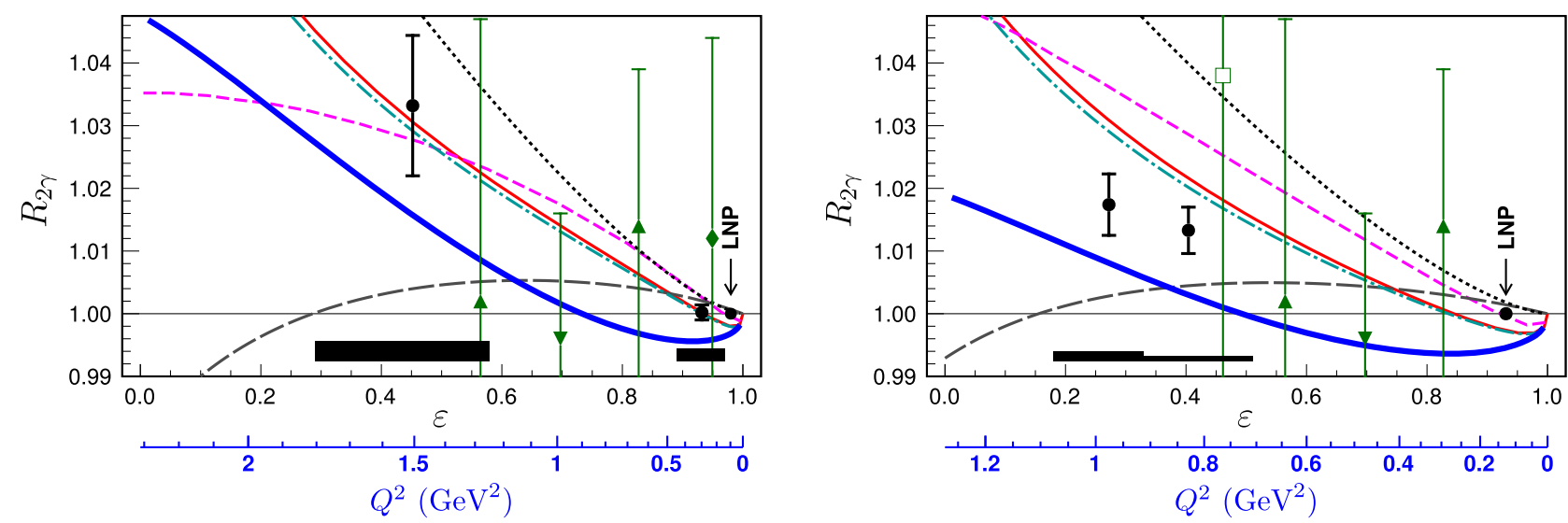

Figure 3.6: $R_{2 \gamma}$ as a function of $\varepsilon$ and $Q^{2}$. The black circles are the VEPP-3 data points, while the other data points are from the early experiments [97-99, 101]. The calculation curves are cyan dash-dotted [52], red solid line [31], blue solid [35], gray long-dash [119] magenta short-dash [120], and black dots [121]. Figure adapted from Ref. [105].

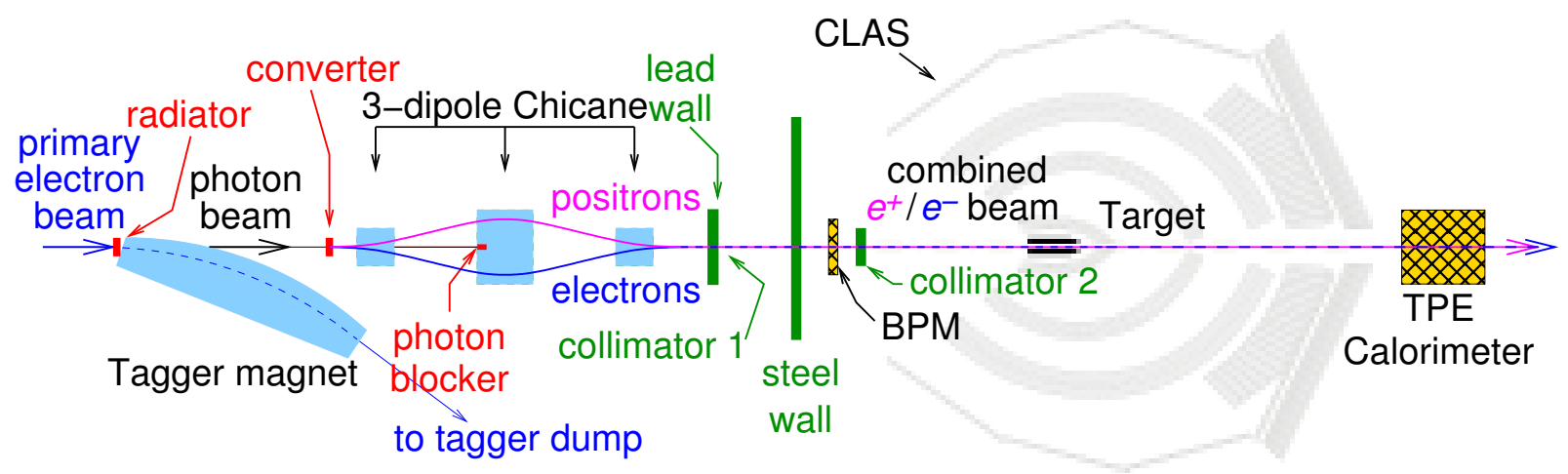

Figure 3.7: Beamline sketch for the CLAS TPE experiment. The chicane bends the electron and positron trajectories in the horizontal plane, rather than the vertical plane as shown in the figure. The electron and positron directions are selected by the chicane polarity. The cutaway view of CLAS shows the three regions of drift chambers (DC), the time-of-flight scintillation counts (TOF), the Čerenkov Counters (CC), and the Electromagnetic Calorimeters (EC). The TPE Calorimeter was removable and only placed in the beam for special calibration runs. Drawing is not to scale.

CLAS is a nearly $4 \pi$ acceptance spectrometer divided into six sectors by superconducting coils that produce a toroidal magnetic field in the azimuthal direction. These magnets bent charged particles away or toward the beamline. The polarity of the CLAS magnetic field was flipped periodically during the experiment, thus flipping the tracks of electrons and positrons. Drift chambers (DC), including a set within the magnetic field, measured charged-particle trajectories enabling momentum and scattering-angle determination. Scintillation counters (TOF) measured the charged-particle time of flight, which when combined with momentum measurements provided particle identification. Electromagnetic Calorimeters (EC), which were generally used in typical CLAS experiments to measure total energy for charged and neutral particles, were used in the event read-out trigger.

Using a mixed electron-positron beam allowed the simultaneous detection of $e^{-} p$ and $e^{+} p$ events, thus ensuring an identical experimental configuration for both. However, a number of unique challenges resulted. In order to produce sufficient luminosity, the primary electron beam ran at a much higher current (between 110 and $140 \mathrm{nA}$ ) than previous CLAS experiments leading to a large radiation background from the radiator and the beam dump. The process of converting the photon beam to $e^{+} / e^{-}$pairs also produced a large radiation background. Extensive shielding was required around the beam dump, the chicane, and in front of CLAS to prevent the detectors from becoming overwhelmed 
with background.

The symmetry of the spatial and energy spectrum of the two lepton beams was determined by a scintillating fiber beam monitor (BM) and a removable lead/scintillator calorimeter. The BM was located at the upstream entrance to CLAS and was used to adjust the chicane field settings to produce maximally overlapping beams. The calorimeter was inserted into the beam every time a the chicane polarity was reversed (thus flipping which side of the chicane the electrons and positrons passed through) to determine relative energy distributions. Individual electron or positron energy distributions were measured by blocking one side of the chicane.

The experiment did not measure absolute cross sections because there was no way to measure the absolute flux of leptons on the target. Instead, the experiment relied upon the fact that both types of leptons are produced in equal numbers at the relevant energies and that differences in various particle acceptances will cancel by forming multiple ratios. For a given torus polarity, $t= \pm$, and chicane polarity, $c= \pm$, the ratio of detected elastically-scattered positrons, $N_{t c}^{+}$, and electrons, $N_{t c}^{-}$ was measured:

$$
R_{t c}=\frac{N_{t c}^{+}}{N_{t c}^{-}}
$$

This cancels out any proton acceptance and detector efficiency factors for the two different lepton events. The yield $N_{t c}^{ \pm}$is proportional to the elastic-scattering cross section, $\sigma^{ \pm}$(here \pm refers to the lepton charge), the lepton-charge-related detector efficiency and acceptance function, $f_{t}^{ \pm}$, as well as the luminosity for a given chicane polarity, $L_{c}^{ \pm}$, so that

$$
R_{t c}=\frac{\sigma^{+} f_{t}^{+} L_{c}^{+}}{\sigma^{-} f_{t}^{-} L_{c}^{-}}
$$

The square root of the product of measurements done with both torus polarities but a fixed chicane polarity leads to

$$
R_{c}=\sqrt{R_{+c} R_{-c}}=\sqrt{\frac{N_{+c}^{+}}{N_{+c}^{-}} \frac{N_{-c}^{+}}{N_{-c}^{-}}}=\sqrt{\frac{\sigma^{+} f_{+}^{+} L_{c}^{+}}{\sigma^{-} f_{+}^{-} L_{c}^{-}} \frac{\sigma^{+} f_{-}^{+} L_{c}^{+}}{\sigma^{-} f_{-}^{-} L_{c}^{-}}}=\frac{\sigma^{+}}{\sigma^{-}} \frac{L_{c}^{+}}{L_{c}^{-}},
$$

where it was assumed that $f_{+}^{+}=f_{-}^{-}$and $f_{-}^{+}=f_{+}^{-}$. That is, the unknown detector efficiency and acceptance functions for positrons cancel those for electrons when the torus polarity is switched and are expected to cancel out in this double ratio.

By reversing the chicane current the spatial positions of the oppositely charged lepton beams is swapped so that $L_{+}^{+}=L_{-}^{-}$and $L_{-}^{+}=L_{+}^{-}$. Then taking the square root of the product of the double ratios defined in Eq. (3.15) leads to

$$
R=\sqrt{R_{++} R_{-+} R_{+-} R_{--}}=\sqrt{\frac{N_{++}^{+}}{N_{++}^{-}} \frac{N_{-+}^{+}}{N_{-+}^{-}} \frac{N_{+-}^{+}}{N_{+-}^{-}} \frac{N_{--}^{+}}{N_{--}^{-}}}=\sqrt{\frac{\sigma^{+} L_{+}^{+}}{\sigma^{-} L_{+}^{-}} \frac{\sigma^{+} L_{-}^{+}}{\sigma^{-} L_{-}^{-}}}=\frac{\sigma^{+}}{\sigma^{-}},
$$

thus eliminating any flux-dependent differences between the two lepton beams.

Though this process in principle eliminates any acceptance differences between $e^{+} p$ to $e^{-} p$ events, further corrections were necessary to account for detection inefficiencies and the fact that the experiment ran with a "minitorus". The minitorus was a fixed polarity magnet system that bent low-energy Møller electrons in the forward direction and out of the detector. These remaining acceptance differences were accounted for by an algorithm that kept events only if the oppositely-charged lepton event also would have been within the detector acceptance and also by a Monte Carlo simulation that included the minitorus field and produced a residual acceptance correction factor.

The identification of elastic events for this experiment relied upon the fact that elastic scattering kinematics are overdetermined when the momenta and scattering angles of both the scattered leptons and protons are experimentally measured. The analysis utilized a co-planarity cut $(\delta \phi)$ and a series of 

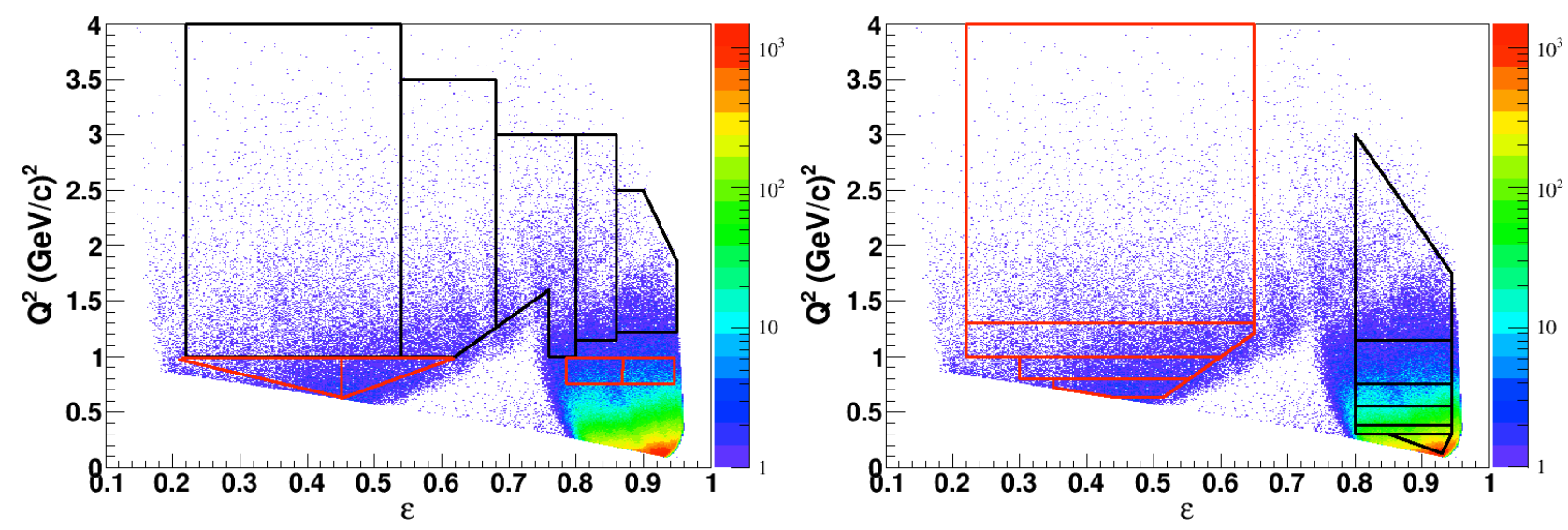

Figure 3.8: Data binning in $Q^{2}$ and $\varepsilon$ overlaid on positive torus $e^{+} p$ events. The left plot shows the two sets of bins for the $\varepsilon$ dependence (red and black boxes for $\left\langle Q^{2}\right\rangle=0.85$ and $1.45 \mathrm{GeV}^{2}$, respectively), while the right plot shows the two binning choices for the $Q^{2}$ dependence (red and black boxes for $\langle\varepsilon\rangle=0.45$ and 0.85 , respectively.)

three other cuts related to the kinematics of the events. The incident lepton beam energy was unknown but was reconstructed from the measured kinematic variables.

Though background was very small (less than $1 \%$ of the signal) for forward-scattered lepton events, at large angles a few percent background was present in the final data set. The background was modelled by taking events from the sideband of one of the energy distributions and were projected onto the $\delta \phi$ distribution. It was found to be Gaussian in shape so a Gaussian background model was used to removed background from all data bins.

The kinematic coverage of $Q^{2}$ versus $\varepsilon$ for the CLAS experiment is shown in Fig. 3.8 . The hole in the distribution at $\varepsilon \approx 0.7$ and lower values of $Q^{2}$ is due to the trigger used in the experiment, which required one particle track hitting the forward TOF and the EC. Events where neither particle had a lab-frame scattering angle of less than about $45^{\circ}$ did not trigger the CLAS readout. The trigger hole is largest for $e^{+} p$, positive torus events, which ultimately limited the kinematic coverage. Data near the edges of the distributions, where the acceptance for in-bending and out-bending particles vary rapidly, were not included in the analysis. This binning choice led to some overlap in the data bins so not all of the published results are independent.

The measured $e^{+} p$ to $e^{-} p$ ratio as defined in Eq. (3.16) and corrected for additional acceptance effects required correction for radiative effects. The largest contribution is from charge-even terms, which are the same for positrons and electrons and acts as a dilution factor in the measured ratio. The charge-odd terms include both the TPE contribution and the interference between real photon emission from the proton and from the leptons (bremsstrahlung interference). These corrections were determined by simulating radiative effects as described in Ref. [123], using the "extended peaking approximation." Simulations were run for electron-proton scattering and again for positron-proton scattering. The average of the two simulation yields give the charge even correction $\delta_{\text {even }}$ and the ratio of these yields give the charge asymmetry that corresponds to the no-TPE limit of Eq. (3.1). The difference between uncorrected and corrected results varied from 0.003 at high $\varepsilon$ and low $Q^{2}$ to 0.034 at low $\varepsilon$ and high $Q^{2}$. The quoted uncertainties in the radiative corrections were a scale uncertainty of roughly $0.3 \%$ and a point-to-point uncertainty of $15 \%$ of the correction and were generally small compared to the statistical and other systematic uncertainties.

The total instrumental systematic uncertainties for the ratio $R_{2 \gamma}$ for this experiment varied between 0.0042 and 0.0187 . These are typically dominated by effects of the kinematic cuts and variations of the measured ratio from sector to sector, indicative of acceptance and efficiency variations. For comparison, statistical uncertainties varied between 0.0067 and 0.0125 .

The results of the experiment as presented in Ref. [20] show an $\varepsilon$ dependence at $\left\langle Q^{2}\right\rangle \approx 0.85$ 


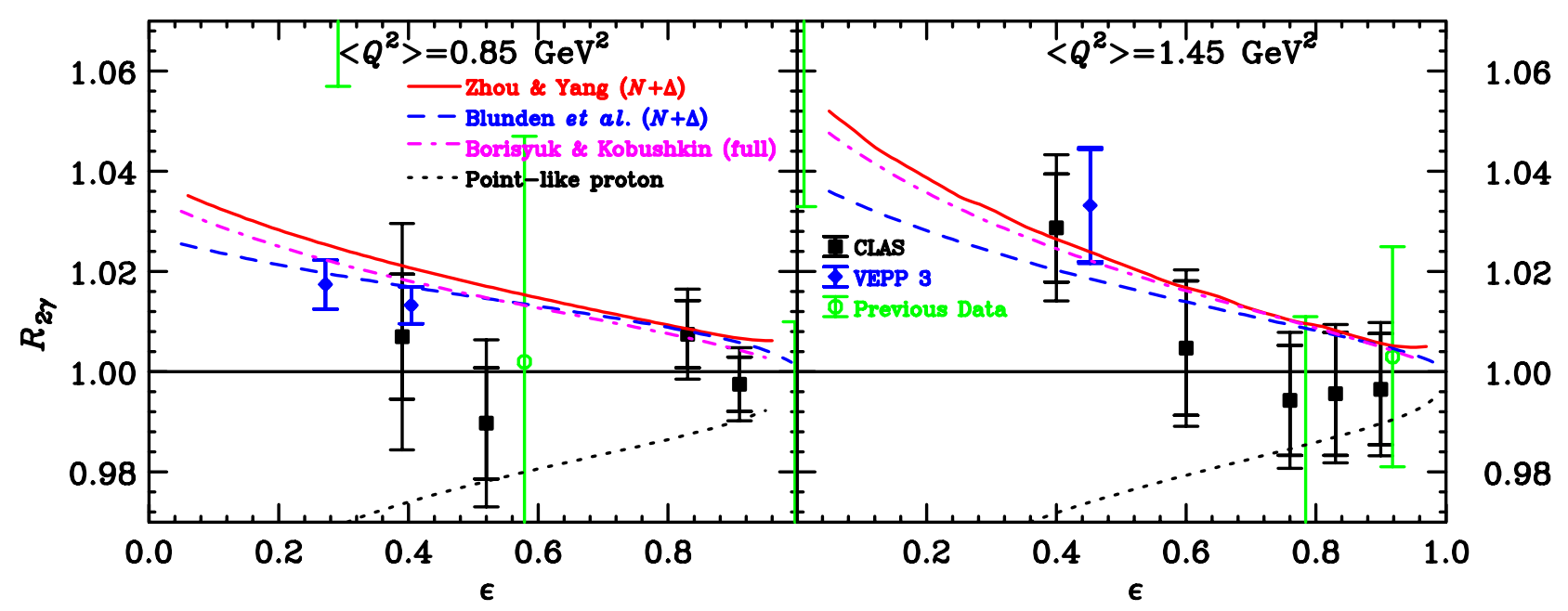

Figure 3.9: $R_{2 \gamma}$ as a function of $\varepsilon$ at $Q^{2} \approx 0.85 \mathrm{GeV}^{2}$ (left) and $1.45 \mathrm{GeV}^{2}$ (right) extracted from the measured ratio of $e^{+} p / e^{-} p$ cross sections corrected for both $\delta_{\mathrm{b}, e p}$ and $\delta_{\text {even. }}$. The filled black squares show the results of the CLAS experiment [20], while the filled blue diamonds are from VEPP-3 [105] at similar kinematics. The open green circles show the previous world data at $0.7 \leq Q^{2} \leq 1.0 \mathrm{GeV}^{2}$ and $1.2 \leq Q^{2} \leq 1.53 \mathrm{GeV}^{2}$ in the left and right plots, respectively [66, 97, 99, 101]. The error bars reflect statistical and point-to-point systematic uncertainties combined. The line at $R_{2 \gamma}=1$ is the limit of no TPE. The solid red curve shows the calculation by Zhou and Yang [44] including $N+\Delta$ intermediate states. The dashed blue curve shows the calculation by Blunden et al. [31, 54] including $N+\Delta$ intermediate states (full). The dot-dashed purple curve shows the calculation by Borisyuk and Kobushkin [58]. The black dot-dashed line shows the calculation of TPE effects on a structureless point proton [2].

and $1.45 \mathrm{GeV}^{2}$. These results, along with results from the VEPP-3 experiment at similar $Q^{2}$ values are shown in Fig. 3.9 as well as predictions from Refs. [54, 58], along with the no TPE limit and a structureless proton model [2]. At the time the CLAS results were submitted for publication the OLYMPUS results were not available. The conclusions drawn were that that the CLAS and VEPP-3 results at $Q^{2}=1.45 \mathrm{GeV}^{2}$ showed a "moderate" increase in $R_{2 \gamma}$ with decreasing $\varepsilon$, while at $Q^{2}=0.85$ no clear change with $\varepsilon$ is apparent.

Figure 3.10 shows the $Q^{2}$ dependence of the CLAS data, again with the VEPP-3 results at similar values of $\varepsilon$. Also included in the figure for the high $\varepsilon$ data is a single data point from the CLAS TPE test run [95], which was primarily a proof of principle experiment that ultimately had a large uncertainty due to data being taken with only a single chicane setting leading to a large normalization uncertainty. The seven data points from that experiment were taken at an average $Q^{2}$ of $0.206 \mathrm{GeV}^{2}$ and $0.830 \leq \varepsilon \leq 0.942$. These seven data points were averaged together in the single point presented in the figure. The CLAS and VEPP-3 data showed only a hint of a rise with $Q^{2}$ at the lower value of $\varepsilon$ and no indication of a change with $Q^{2}$ at the higher value of $\varepsilon$.

The CLAS paper provided a global analysis that included both the CLAS data and the VEPP-3 data but excluded the previous world data due to their large uncertainties. The global analysis compared 12 independent CLAS data points and the four non-normalization data points to the hadronic calculations of Refs. [31, 44], the no-TPE assumption, and the calculation based on a structureless proton [2]. These data are in good agreement with the hadronic calculations of Refs. [31, 44] but of insufficient precision to make any definitive distinction between them. However, the CLAS and VEPP-3 data exclude the no-TPE hypothesis at the $5.3 \sigma$ level, and rule out the point-proton result at the $\sim 25 \sigma$ level, which is essentially equivalent to the $Q^{2}=0$ limit. A summary of the CLAS global analysis is shown in Table 3.1. Again, as we shall discuss later, the inclusion of the OLYMPUS results leads to a rather different conclusion.

The CLAS results also included a corrected Rosenbluth separation based upon a linear fit of all of the data at $Q^{2} \approx 1.45 \mathrm{GeV}^{2}$ shown in Fig. 3.9. This fit constrained the line to go to $R_{2 \gamma}=1$ at $\varepsilon=1$. 


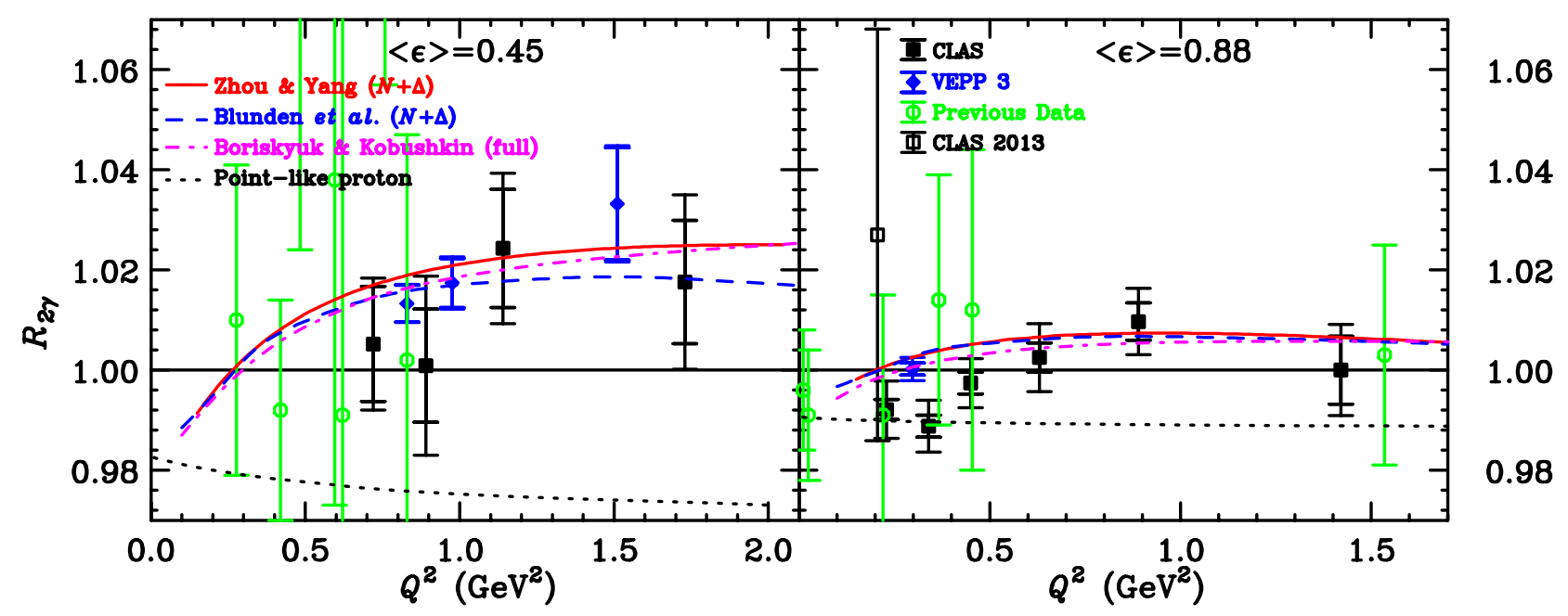

Figure 3.10: $R_{2 \gamma}$ as a function of $Q^{2}$ at $\varepsilon \approx 0.45$ (left) and 0.88 (right). The data and curves are the same as in Fig. 3.9 with an additional point from the CLAS TPE test-run experiment [95] (black open square). The open green circles show the previous world data $[66,96,97,99,101,103]$ at $0.2 \leq \varepsilon \leq 0.7$ and $0.7 \leq \varepsilon \leq 0.95$ in the left and right panels, respectively.

\begin{tabular}{llc}
\hline TPE calculation & $\chi_{\nu}^{2}$ & Confidence level $(\%)$ \\
\hline Blunden et al. $(N)[31]$ & 1.23 & 23 \\
Zhou \& Yang $(N)[44]$ & 1.27 & 20 \\
Zhou \& Yang $(N+\Delta)[44]$ & 1.19 & 27 \\
$\delta_{\gamma \gamma}=0$ (No TPE) & 2.32 & 0.2 \\
Point-proton calculation & 7.38 & $3.0 \times 10^{-15}$ \\
\hline
\end{tabular}

Table 3.1: Comparison of the 16 CLAS and VEPP-3 data points to various TPE calculations showing the reduced $\chi^{2}$ value and the confidence level.

From this fit, $\delta_{\gamma \gamma}$ was determined as a function of $\varepsilon$ and applied as a correction to the reduced crosssection data of Andivahis et al. [124] according to Eq. (2.23). This TPE correction changed the proton form factor ratio, $R=\mu_{p} G_{E} / G_{M}$, from the original value of $0.910 \pm 0.060$ to $0.820 \pm 0.044$. This brings it into good agreement with the polarization transfer result of $0.789 \pm 0.042$ at $Q^{2}=1.77 \mathrm{GeV}^{2}[15]$.

The conclusion of this paper is that the CLAS and VEPP-3 data combined indicate a non-zero TPE effect that is consistent with models that provide a TPE correction that generally account for the Rosenbluth and polarization transfer discrepancy.

\subsection{The OLYMPUS experiment}

The OLYMPUS experiment was designed to measure the ratio between positron-proton and electronproton elastic scattering over a broad angular range, $25^{\circ}<\theta<75^{\circ}$, with a precision of around $1 \%$. Only a brief description of the experiment will be given here. A full description can be found in [125].

OLYMPUS ran on the DORIS positron/electron storage ring at the DESY laboratory, Hamburg, Germany. Data were collected in two periods for approximately three months in total during 2012 before DORIS was shutdown. The lepton beam energy was nominally $2.01 \mathrm{GeV}$ with currents up to $70 \mathrm{~mA}$. The lepton beam species (electrons or positrons) was changed daily. A total integrated luminosity of $\sim 4.5 \mathrm{fb}^{-1}$ was collected.

The stored lepton beam passed through a windowless, unpolarized, hydrogen gas target [126] internal to the DORIS storage ring. The typical areal target density was $\sim 3 \times 10^{15}$ atoms $/ \mathrm{cm}^{2}$. 


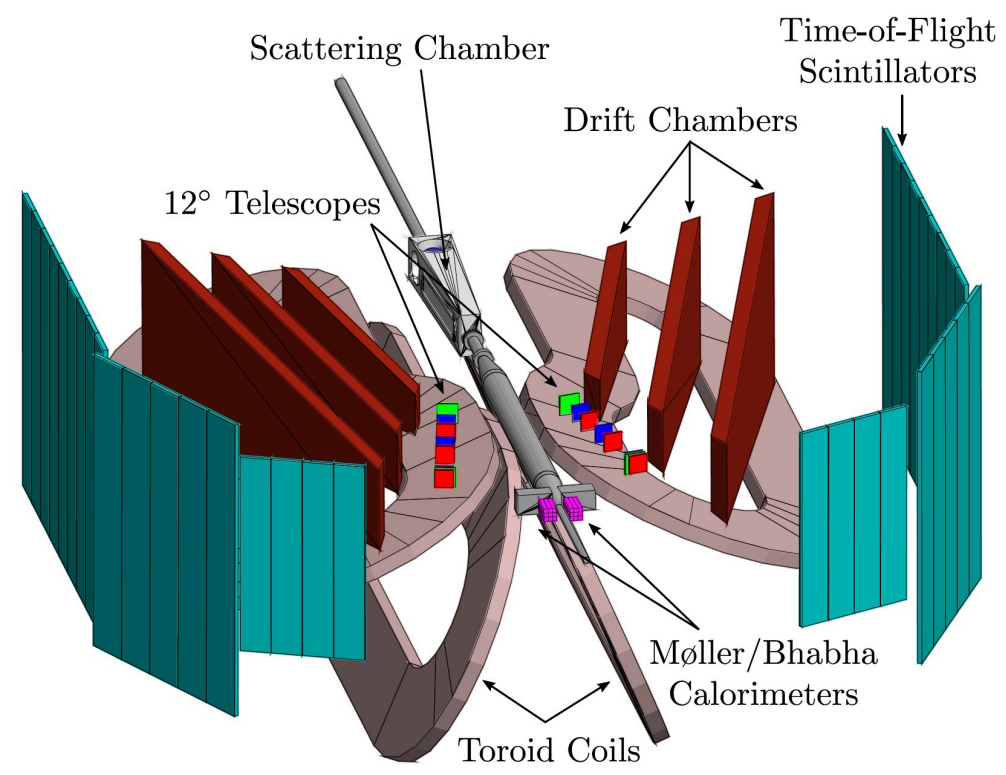

Figure 3.11: Schematic representation of the OLYMPUS detector with the top four toroid coils removed to reveal the two horizontal, instrumented sectors. Note the drift chambers are shown as three separate chambers in each sector but in actuality were enclosed in a single gas volume.

The OLYMPUS detector (see Fig. 3.11) was based on the former MIT-Bates BLAST detector [127]. This consisted of an eight sector, toroidal, magnetic spectrometer with the two horizontal sections instrumented with large acceptance $\left(20^{\circ}<\theta<80^{\circ},-15^{\circ}<\phi<15^{\circ}\right)$ drift chambers (DC) for particle tracking and walls of time-of-flight (ToF) scintillator bars to trigger the data acquisition system and for particle identification. To a good approximation the detector system was left-right symmetric, and this redundancy was used as a cross check by analyzing and comparing the result determined when the lepton scattered into the left sector with the result when the lepton scattered into the right sector.

Two new detector systems were designed and built to monitor the luminosity. Symmetric Møller / Bhabha calorimeters [128] (SYMB) consisting of $3 \times 3$ arrays of $\mathrm{PbF}_{2}$ crystals behind lead collimators were situated at $1.29^{\circ}$ left and right of the beam axis and approximately $3 \mathrm{~m}$ downstream from the target. There were also two detector telescopes of three triple GEM detectors interleaved with three MWPC detectors mounted to the left and right drift chambers at $12^{\circ}$. The $12^{\circ}$ telescopes also had plastic scintillators, front and back, with SiPM readout used in coincidence to trigger the readout of the GEM and MWPC tracking detectors.

The first level trigger system used left-right coincidences between ToF bars loosely corresponding to $e^{ \pm} p$ elastic scattering angles. The second level trigger required at least one hit in the middle and outer drift chambers of each sector to indicate a potential track. This helped reduce noise events and allowed a higher rate of useful events to be collected.

In 2013, immediately after the experimental data runs, cosmic ray data were collected for one month. Then a complete optical survey of the detector positions was made and the magnetic field was mapped throughout the tracking volume [129] and the volume between the scattering chamber and the SYMB.

\subsubsection{Luminosity}

The integrated luminosity for each beam species was monitored by four independent methods using: the slow control information, the $12^{\circ}$ telescopes, the SYMB, and a multi-interaction event (MIE) method that also used the SYMB calorimeters.

The slow control system monitored and recorded the beam current, beam position and slope, and the flow of gas into the target cell in addition to numerous other parameters. A detailed molecular flow 
simulation converted the gas flow rate into the target areal density. Taking the product of the beam current and target density yielded a $5 \%$ absolute luminosity measurement and a $2 \%$ relative measure between beam species that was available online for quick analyses.

The $12^{\circ}$ MWPC detectors tracked leptons elastically scattered over a small range of angles around $12^{\circ}$ in both the left and right sectors in coincidence with the recoil proton tracked in the DC and ToF around $72^{\circ}$. Combined with the MC simulation of $e^{ \pm} p$ elastic scattering and assuming a small contribution from two-photon effects, a luminosity determination was possible at the level of $1 \%$ every 20 minutes and a statistical accuracy on the order of $0.01 \%$ over the whole experiment. Including systematic uncertainties, the $12^{\circ}$ system provided a $2.4 \%$ absolute and a better than $0.5 \%$ relative luminosity determination. The GEM detector readout was not used in tracking or luminosity measurements at $12^{\circ}$ though it was useful in calibrating and aligning the MWPC.

The symmetric Møller/Bhabha calorimeters should have provided a fast and high precision measurement of the luminosity. Unfortunately the steep and differing slopes of the Møller and Bhabha cross sections made it extremely sensitive to the exact geometry and alignment of beam position, beam slope, and the collimators in front of the calorimeters. Ultimately the relative luminosity was limited to an uncertainty of $\pm 3 \%$. In addition, time-dependent readout issues were encountered which were problematic.

However, the systematic and electronics issues with the planned Møller / Bhabha measurements could be overcome by comparing the relative rates for the lepton-lepton coincidences with the rate for detecting a $\sim 2 \mathrm{GeV}$ lepton from lepton-proton elastic scattering in one of the calorimeters in coincidence with the lepton-lepton coincidence [130]. This multi-interaction event (MIE) method produced a $0.3 \%$ relative uncertainty in luminosity between beam species.

The slow control, $12^{\circ}$, and MIE methods for determining the luminosity were all in excellent agreement lending support for the measurement of the luminosity. The MIE was chosen to normalize the analysis because it had the smallest uncertainty. This had the additional advantage that the $12^{\circ}$ detector system could be used to measure the ratio of $\sigma_{e^{+} p} / \sigma_{e^{-} p}$ at $12^{\circ}$ providing a measure of twophoton exchange contributions at low $Q^{2}$ (high $\varepsilon$ ) where two-photon exchange is generally expected to be small.

\subsubsection{Radiative corrections}

Radiative corrections are an important step in analyzing any electron scattering experiment and it is important to include all the first order processes in calculating the radiative corrections.

In the OLYMPUS experiment radiative corrections can not be simply applied to the measured cross section. OLYMPUS measured the lepton and proton in coincidence over a broad kinematic range where the acceptance, efficiency, and energy resolution vary as a function of $Q^{2}$. Therefore it was necessary to build a radiative generator that could be used in the Monte Carlo simulation of the experiment. This was done in parallel with the radiative generator developed by the VEPP-3 group [118] and was used to cross-check both calculations. The OLYMPUS radiative generator had numerous options to select the proton form factor (point-like, Kelly, Bernauer, etc.), soft-photon prescription (Mo-Tsai, Maximon-Tjon, etc.), vacuum polarization calculation, and whether or not to use exponentiation. The cut-off energy in OLYMPUS was typically a few percent, and beyond this radiative corrections were explicitly calculated.

It is important to note the significance of the soft TPE contributions at these energies. Figure 3.12 compares four calculations of the radiative corrections relative to the Born result as a function of $\varepsilon$. The difference between Mo-Tsai and Maximon-Tjon is not significant. But as $\varepsilon$ decreases to 0.45 the effect increases quickly from $\sim 1 \%$ to $5-7 \%$, and the non-exponentiated $\left(\alpha^{3}\right)$ effect is about $\sim 1 \%$ larger than the exponentiated contribution. 


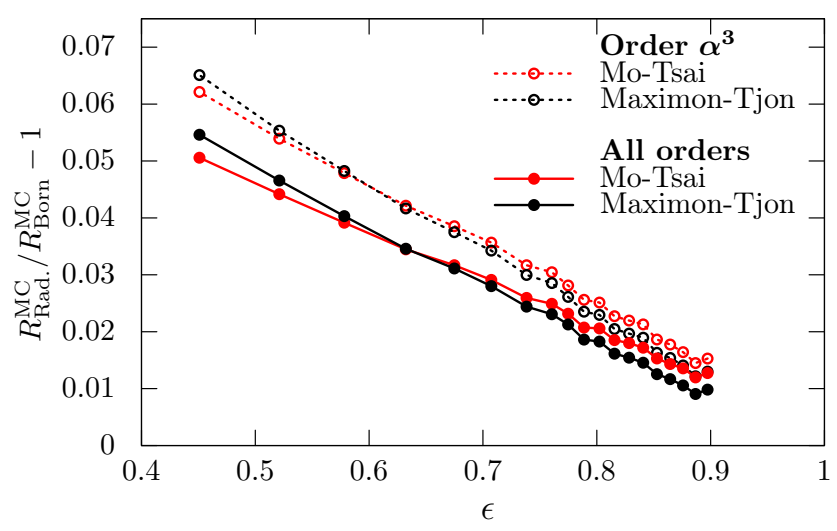

Figure 3.12: Effect of radiative corrections relative to the Born result for the two prescriptions Mo-Tsai [7] and MaximonTjon [8] and for the $\alpha^{3}$ (non-exponentiated) or exponentiated calculation as a function of $\varepsilon$.

\subsubsection{Analysis}

The analysis presented here is a combination of the results of four independent analyses (three $\mathrm{PhD}$ theses [130-132] and an analysis performed by Jan Bernauer). The results are available at [107]. Two other theses are nearing completion $[133,134]$ and these will be incorporated into a longer paper with a more thorough description of each analysis. Each analysis was developed independently and their results are highly compatible. For the results shown here and published in [107] we have averaged the four results without weighting (statistics for each were comparable) and the spread in the results was included as a point-to-point systematic uncertainty.

The analyses to date are based on a subset of the total recorded data by selecting runs with optimal running conditions (without tripped channels, etc). These correspond to about $3.2 \mathrm{fb}^{-1}$ of integrated luminosity.

Track reconstruction began with a pattern matching algorithm to identify potential tracks and to obtain initial estimates for the track parameters. Then two different tracking algorithms were employed to fit each track candidate to optimize the track vertex, scattering angles, and momentum. Both algorithms produced very similar results. The final tracked data sets consisted of candidate track momentum, polar and azimuthal angles, $z$ position in the target, charge, track path length, time to time-of-flight detector, and energy deposited in the ToF.

Starting from the same tracked data set each analysis performed an independent analysis. For each analysis this included a series fiducial cuts to select good tracks. This was followed by applying loose cuts on all combinations of tracks in an event to select pairs of tracks consistent with elastic $e^{ \pm} p$ scattered events and to reduce background events. Further cuts were then applied to select the final $e^{ \pm} p$ events. The resulting events were binned in a common selection of $Q^{2}$ bins reconstructed from the proton scattering angle to minimize fake asymmetries (the two lepton charges bend differently and could have different errors in the reconstructed kinematics). In each bin, the background was subtracted. At forward angles (low $Q^{2}$ ) the background was negligible increasing to approximately $20 \%$ at backward angles (high $Q^{2}$ ). This background fraction was roughly the same for all analyses and also the same for both electron and positron runs. The number of events in each $Q^{2}$ bin after background subtraction was collected for both electron and positron beams.

A complete Monte Carlo (MC) simulation of the detector and experiment was also made with full digitization to produce MC data in exactly the same format as the real data. This allowed the acceptances, efficiencies, and resolutions of the DC, ToF, and $12^{\circ}$ detectors to be simulated in the MC and compared with the real data.

A radiative event generator was developed specifically for OLYMPUS (see section 3.7.2). This generated $e^{ \pm} p$ events (including inelastic processes) weighted by the scattering cross sections. Since the radiative corrections depend on the proton structure and various radiative correction prescriptions 


\begin{tabular}{ll}
\hline Type of contribution & Uncertainty in $R_{2 \gamma}$ \\
\hline Correlated contributions & \\
Beam energy & $0.04-0.13 \%$ \\
MIE luminosity & $0.36 \%$ \\
Beam and detector alignment & $0.25 \%$ \\
Uncorrelated contributions & \\
Tracking efficiency & $0.20 \%$ \\
Elastic selection and background subtraction & $0.25-1.17 \%$ \\
\hline
\end{tabular}

Table 3.2: Contributions to the OLYMPUS systematic uncertainty in $R_{2 \gamma}$.

each of these effects were carried as separate cross section weights. Carrying the various cross section weights throughout the simulation allowed their effect to be studied without having to regenerate and re-track the MC for each.

The generated events were propagated through the detector simulation using GEANT4. More MC data were produced than real data to reduce systematic uncertainties due to the MC simulation. The $\mathrm{MC}$ used slow control information like the lepton beam energy, position, slope, and instantaneous luminosity to match the data as closely as possible on a run-by-run basis. The MC data were then analyzed using the same code, event selection, and cuts as used on the real data.

To obtain the ratio between positron-proton and electron-proton elastic scattering as a function of $Q^{2}$ (or $\varepsilon$ ), $R_{2 \gamma}\left(Q^{2}\right)=\sigma_{e^{+} p}\left(Q^{2}\right) / \sigma_{e^{-} p}\left(Q^{2}\right)$, we take the luminosity weighted ratio of the number of events for both data and MC for each $Q^{2}$ bin:

$$
R_{2 \gamma}=\frac{N_{\exp }\left(e^{+}\right)}{N_{\exp }\left(e^{-}\right)} / \frac{N_{\mathrm{MC}}\left(e^{+}\right)}{N_{\mathrm{MC}}\left(e^{-}\right)} .
$$

Note that we do not correct the yield from data for efficiencies, acceptances, or radiative effects. Rather this is all included and corrected through the complete Monte Carlo simulation.

In addition to statistical uncertainties there are various uncorrelated systematic uncertainties that vary from bin to bin and correlated systematic uncertainties common to all bins. The systematic uncertainties are given in table 3.2 .

The final results for $R_{2 \gamma}$ for OLYMPUS are available in [107] and are shown in Fig. 3.13. The results are plotted with the statistical and systematic uncertainties together with the theoretical calculations from Blunden for $N$ and $N+\Delta$ [54] and the predictions from the phenomenological fit to the existing form factor data by Bernauer [35]. The plotted results are with the radiative corrections to all orders using the convention of Mo-Tsai [7] for compatibility with the CLAS and VEPP-3 results.

The OLYMPUS results are in general less than unity at high $\varepsilon$ gradually rising to around $2 \%$ at $\varepsilon=0.456$. The OLYMPUS results are systematically lower than the theoretical calculations of Blunden but in reasonable agreement with the predictions of Bernauer's phenomenological fit. This implies that perhaps the theoretical calculations that account for the discrepancy in the form factor ratio at higher $Q^{2}$ do not extend to this relatively low $Q^{2}$ region or that other effects need to be taken into account. Bernauer's fit that includes low $Q^{2}$ measurements agrees with the data better.

Bernauer's model [35] was a fit to all the available form factor data including the polarization data (1866 data points). For the unpolarized data he extracted cross section data and redid the radiative corrections to standardize the treatment. The Mainz data, included in the fit, were the largest and most consistent set of data and extended to the smallest $Q^{2}$ values available. To include the polarization measurements he modelled the hard two-photon exchange contribution as the Feshbach correction of Eq. (2.24) plus $\delta_{\text {hard }}$ using the parametrization:

$$
\delta_{\text {hard }}=-(1-\varepsilon) a \log \left(b Q^{2}+1\right),
$$




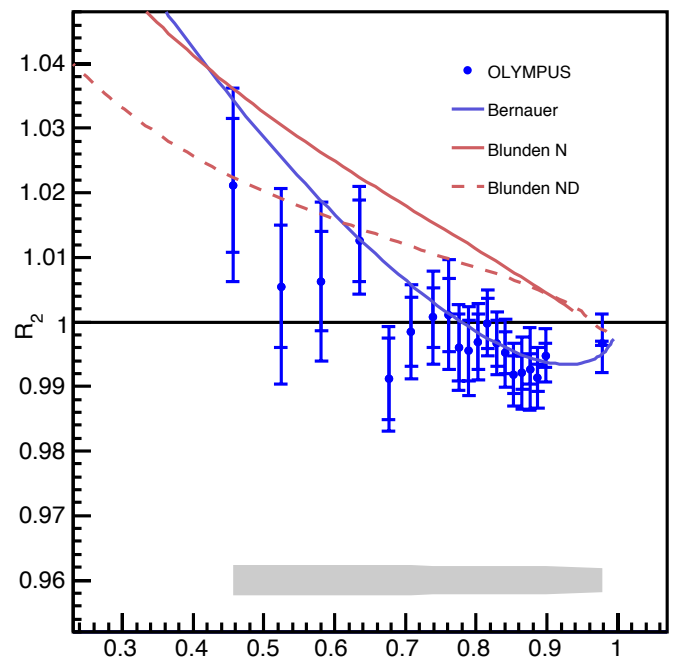

Figure 3.13: OLYMPUS results for $R_{2 \gamma}$ using the exponentiated Mo-Tsai radiative corrections plotted as a function of $\varepsilon$. The results are plotted with statistical uncertainties (inner error bars) and uncorrelated systematic uncertainties (outer error bars). The correlated uncertainty is represented by the gray bar at the bottom of the figure. Theoretical calculations from Blunden [54] for $N$ and $N+\Delta$ and the phenomenological fit to the form factor data from Bernauer [35] is also shown.

where $a$ and $b$ were included in the fit parameters. The fit found $a=0.06894$ and $b=0.3947 \mathrm{GeV}^{-2}$. The final fit was the product of a spline interpolant and the standard dipole form. The spline used knots at $Q^{2}$ values of: $0.0,0.25,0.5,0.75,1.0,1.5,3.0,5.0,10.0$, and $40.0 \mathrm{GeV}^{2}$, and achieved $\chi_{\nu}^{2}=2151.72 / 1866=1.153$.

\subsection{Comparison of recent experiments and models}

The kinematic coverage in $Q^{2}$ versus $\varepsilon$ of the three new experiments, VEPP-3, CLAS, and OLYMPUS is shown in Fig. 3.14, which shows the binning used by each experiment. Both VEPP-3 and OLYMPUS ran with monoenergetic beams so their bins are sums over angle ranges leading to a correlated variation over $Q^{2}$ and $\varepsilon$. CLAS had a range of beam energies at much lower integrated luminosity and thus summed bins over ranges in both $Q^{2}$ and $\varepsilon$. The combined data sets are shown in Table 3.3.

The experiments measured few points with the same kinematics so a direct comparison of all of the the data simultaneously would not be appropriate. A plot of $R_{2 \gamma}$ versus $\varepsilon$ or versus $Q^{2}$ would hide the dependency on the other variable. One way to compare the data is to plot each data point's difference from a given model, $R_{2 \gamma}^{\text {data }}-R_{2 \gamma}^{\text {calc }}$, since the model can be calculated at the specific kinematics of the measured data. This is shown in Figs. 3.15 and 3.16 at all kinematics compared to the no TPE limit of $R_{2 \gamma}=1$, the $N+\Delta$ models of both Refs. [54, 58], and the parametrization of Bernauer et al. [35]. The VEPP-3 data have been normalized to the model prediction at their luminosity normalization points in each case and we have used the OLYMPUS data with radiative corrections to all orders with the Mo and Tsai method. In the no TPE limit we see that the OLYMPUS data is systematically below zero at large $\varepsilon$. In the comparisons to Blunden and Melnitchouk [54] and Borisyuk and Kobushkin [58] calculations, the CLAS and VEPP-3 data are evenly scattered about zero while the OLYMPUS data systematically fall below zero at nearly all values of $\varepsilon$. The difference of all of the data to the Bernauer parametrization is small except for, perhaps, at central values of $\varepsilon$ where there is a slight systematic negative difference. The plot of $R_{2 \gamma}=1$ versus $Q^{2}$ for the no TPE limit shows a more clear difference between OLYMPUS and the other two data sets.

This does not present the entire picture since the CLAS and OLYMPUS data sets both have scaletype, or normalization uncertainties that would move the entire data sets up or down. To account for the normalization uncertainty we have preformed two separate statistical analyses of the data. In the first we have added the normalization uncertainties of CLAS and OLYMPUS in quadrature to the 


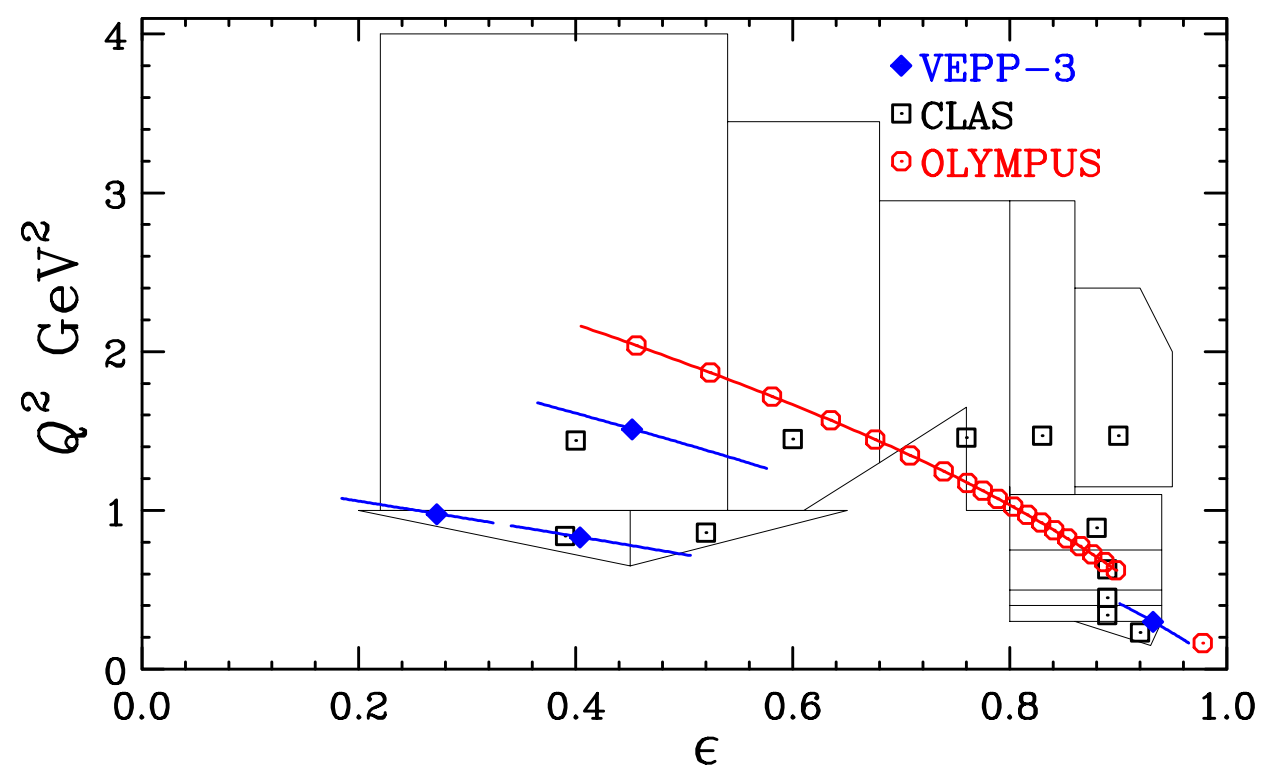

Figure 3.14: Kinematic regions probed by the three two-photon experiments showing the $Q^{2}$ and $\epsilon$ plane. Symbols indicate values at which data points were reported by the respective experiments. The boxed regions show the bins over which the CLAS data are summed and the blue curves indicate the kinematic region over which the VEPP-3 data points are summed to obtain the results at the data points shown by the symbols. The binning of the OLYMPUS data are binned such that the gaps between bins are not visible in the red curve.

statistical and uncorrelated systematic uncertainties. This over inflates the error bars on individual data points but provides an upper limit on the confidence-level agreement between the data and models. We also took into account the fact that not all of the CLAS data presented in Figs. 3.9 and 3.10 are independent. As was done in Ref. [20] we selected the 12 independent data points with the best discriminatory power. These are the ones shown in Fig. 3.14. The VEPP-3 paper does not report a separate normalization uncertainty, rather the normalization depends upon the model to which the data are being compared. A standard $\chi_{\nu}^{2}$ has been calculated for each data set separately and as a whole.

The results of this analysis are shown in the columns labelled "No normalization" of Table 3.4. With this treatment of the normalization uncertainties, the data exclude the the no-TPE hypothesis at the $98 \%$ confidence level, though the OLYMPUS data alone verify this hypothesis at the $89 \%$ confidence level. There is excellent agreement between the collective data set and the Bernauer parametrization, and both the hadronic models tested are excluded at greater than the $96 \%$ confidence level. We stress that one should not read too much into these confidence levels because the error bars on the data points are inflated.

In our second treatment of the normalization uncertainties we have allowed the normalization of the CLAS and OLYMPUS data to float independently but with a penalty determined by the normalization uncertainty of each data set. We select the normalization, $\mathcal{N}$, that minimizes a modified $\chi^{2}$ defined by

$$
\chi^{2}=\sum_{n}\left(\frac{R_{2 \gamma} \mathcal{N}-R_{2 \gamma}^{\text {calc }}}{\delta R_{2 \gamma}^{\text {total }}}\right)^{2}+\left(\frac{\mathcal{N}-1}{\delta R_{2 \gamma}^{\text {norm }}}\right)^{2},
$$

where $R_{2 \gamma}$ is the value reported by the experiments, $\delta R_{2 \gamma}^{\text {total }}$ is the quadrature sum of the statistical and uncorrelated systematic uncertainties, $R_{2 \gamma}^{\text {calc }}$ is the calculated value for a particular model, and $\delta R_{2 \gamma}^{\text {norm }}$ is the normalization uncertainty. The number of degrees of freedom, $\nu$, is then number of data points, $n$, in the set minus one. For CLAS $\nu=11$, and for OLYMPUS $\nu=19$. The analysis for the VEPP-3 data does not change from the "No normalization" analysis. 


\begin{tabular}{|c|c|c|c|c|c|c|c|c|c|}
\hline$Q^{2}$ & $\varepsilon$ & $R_{2 \gamma}$ & $\delta R_{2 \gamma}^{\text {total }}$ & $\delta R_{2 \gamma}^{\text {scale }}$ & $Q^{2}$ & $\varepsilon$ & $R_{2 \gamma}$ & $\delta R_{2 \gamma}^{\text {total }}$ & $\delta R_{2 \gamma}^{\text {scale }}$ \\
\hline VEPP-3 & & & & & $O L Y M P U S$ & & & & \\
\hline 1.510 & 0.452 & 1.0332 & 0.0116 & - & 0.165 & 0.978 & 0.9967 & 0.0046 & 0.0036 \\
\hline 0.298 & 0.932 & 1.0002 & 0.0023 & - & 0.624 & 0.898 & 0.9948 & 0.0042 & 0.0045 \\
\hline 0.976 & 0.272 & 1.0174 & 0.0052 & - & 0.674 & 0.887 & 0.9913 & 0.0047 & 0.0045 \\
\hline 0.830 & 0.931 & 1.0133 & 0.0038 & - & 0.724 & 0.876 & 0.9927 & 0.0064 & 0.0045 \\
\hline$C L A S$ & & & & & 0.774 & 0.865 & 0.9921 & 0.0056 & 0.0045 \\
\hline 0.84 & 0.39 & 1.0070 & 0.0226 & 0.003 & 0.824 & 0.853 & 0.9918 & 0.0049 & 0.0045 \\
\hline 0.86 & 0.52 & 0.9897 & 0.0167 & 0.003 & 0.874 & 0.841 & 0.9952 & 0.0053 & 0.0045 \\
\hline 1.44 & 0.40 & 1.0287 & 0.0146 & 0.003 & 0.924 & 0.829 & 0.9967 & 0.0049 & 0.0045 \\
\hline 1.45 & 0.60 & 1.0047 & 0.0156 & 0.003 & 0.974 & 0.816 & 0.9998 & 0.0051 & 0.0045 \\
\hline 1.46 & 0.76 & 0.9943 & 0.0136 & 0.003 & 1.024 & 0.803 & 0.9969 & 0.0059 & 0.0045 \\
\hline 1.47 & 0.83 & 0.9956 & 0.0138 & 0.003 & 1.074 & 0.789 & 0.9955 & 0.0069 & 0.0045 \\
\hline 1.47 & 0.90 & 0.9965 & 0.0133 & 0.003 & 1.124 & 0.775 & 0.9960 & 0.0066 & 0.0045 \\
\hline 0.23 & 0.92 & 0.9921 & 0.0057 & 0.003 & 1.174 & 0.761 & 1.0011 & 0.0085 & 0.0045 \\
\hline 0.34 & 0.89 & 0.9888 & 0.0052 & 0.003 & 1.246 & 0.739 & 1.0007 & 0.0072 & 0.0045 \\
\hline 0.45 & 0.89 & 0.9974 & 0.0049 & 0.003 & 1.347 & 0.708 & 0.9985 & 0.0073 & 0.0045 \\
\hline 0.63 & 0.89 & 1.0025 & 0.0068 & 0.003 & 1.447 & 0.676 & 0.9912 & 0.0080 & 0.0045 \\
\hline \multirow[t]{4}{*}{0.89} & 0.88 & 1.0097 & 0.0066 & 0.003 & 1.568 & 0.635 & 1.0126 & 0.0084 & 0.0045 \\
\hline & & & & & 1.718 & 0.581 & 1.0063 & 0.0123 & 0.0045 \\
\hline & & & & & 1.868 & 0.524 & 1.0055 & 0.0151 & 0.0045 \\
\hline & & & & & 2.038 & 0.456 & 1.0212 & 0.0150 & 0.0045 \\
\hline
\end{tabular}

Table 3.3: Recent data for $R_{2 \gamma}$, with $Q^{2}$ in $\mathrm{GeV}^{2} . \delta R_{2 \gamma}^{\text {total }}$ is the quadrature sum of statistical and uncorrelated systematic uncertainties. $\delta R_{2 \gamma}^{\text {scale }}$ is the scale-type uncertainty for each experiment.

The statistical results are shown in Table 3.4, and Fig. 3.17 shows the difference between the normalized data values $R_{2 \gamma}^{\text {norm }}=R_{2 \gamma} \mathcal{N}$ and the model predictions $R_{2 \gamma}^{\text {calc }}$ with total uncertainties also scaled by the normalization factor. The combined data still excludes the no-TPE hypothesis but now at the $99.5 \%$ confidence level and there is good agreement with the hadronic models of Refs. [54, 58] with confidence levels of $53 \%$ and $48 \%$, respectively. However, in both cases a large upward normalization is required for the OLYMPUS data that is different from one by nearly $2 \delta R_{2 \gamma}^{\text {norm }}$. The Bernauer parametrization agrees with the data at the $79 \%$ confidence level.

\section{Conclusions and outlook}

New $e^{+} p / e^{-} p$ data from three experiments are now available for $Q^{2}<2.1 \mathrm{GeV}^{2}$. These data are in reasonable agreement with each other except for a steeper $Q^{2}$ dependence in the VEPP-3 results, which largely disappears when compared to calculations that also increase with $Q^{2}$. Collectively, the data sets rule out $\delta_{\gamma \gamma}=0$ at greater than the $95 \%$ confidence level.

Allowing a renormalization of the CLAS and OLYMPUS results achieves reasonable agreement with the calculations of Blunden and Melnitchouk [54] and Borisyuk and Kobushkin [58] and the parametrization of Bernauer et al. [35]. The calculations largely reconcile the Rosenbluth polarization transfer discrepancy of the electromagnetic form factors of the proton. However, to achieve this agreement with the calculations, the CLAS and OLYMPUS results must be shifted by approximately one and two times their respective correlated uncertainties. Without the renormalization only the Bernauer prediction remains in good agreement with the three experimental results and the theoretical calculations are systematically higher than the results. However the $\varepsilon$ and $Q^{2}$ dependence is generally followed by the calculations. 

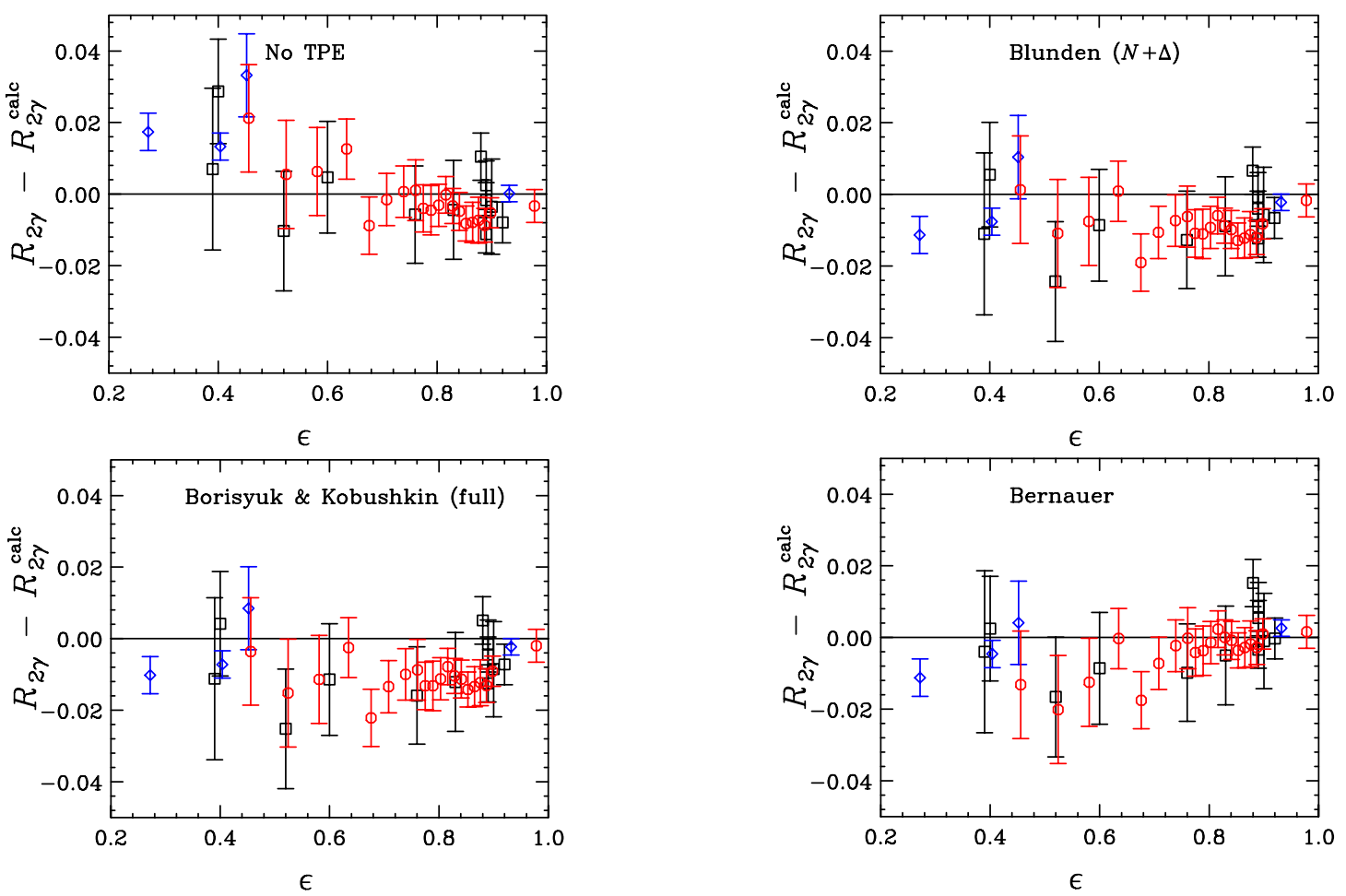

Figure 3.15: Difference between $R_{2 \gamma}$ and model predictions as a function of $\varepsilon$. The blue diamonds are VEPP-3, the black boxes are from CLAS, and the red circles are from OLYMPUS. Error bars reflect the quadrature sum of statistical and uncorrelated systematic uncertainties.
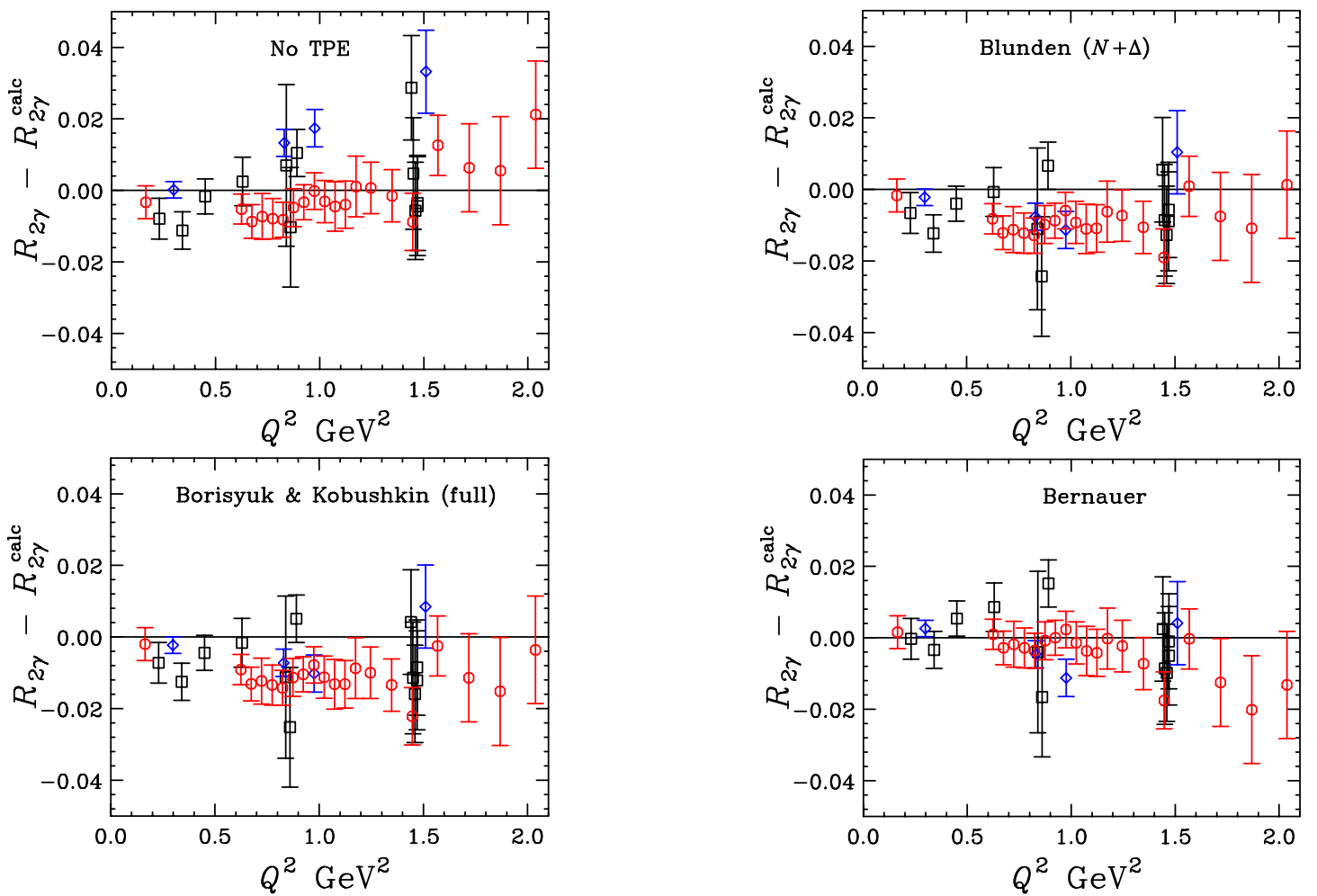

Figure 3.16: Difference between $R_{2 \gamma}$ and model predictions as a function of $Q^{2}$. Data symbols are the same as in Fig. 3.15. 


\begin{tabular}{|c|c|c|c|c|c|c|}
\hline \multirow[b]{2}{*}{ Data set } & \multicolumn{2}{|c|}{ No normalization } & \multicolumn{4}{|c|}{ With normalization } \\
\hline & $\chi_{\nu}^{2}$ & $\nu$ & $\chi_{\nu}^{2}$ & $\nu$ & $\mathcal{N}$ & $\left(\frac{\mathcal{N}-1}{\delta R_{2 \gamma}^{\text {norm }}}\right)$ \\
\hline \multicolumn{7}{|l|}{ Model: $\delta_{\gamma \gamma}=0$} \\
\hline VEPP-3 & 7.97 & 4 & 7.97 & 4 & - & - \\
\hline CLAS & 0.99 & 12 & 1.25 & 11 & 1.0012 & 0.40 \\
\hline OLYMPUS & 0.64 & 20 & 0.68 & 19 & 1.0034 & 0.76 \\
\hline All & 1.57 & 36 & 1.73 & 34 & - & - \\
\hline \multicolumn{7}{|c|}{ Model: Blunden \& Melnitchouk [54] } \\
\hline VEPP-3 & 2.62 & 4 & 2.62 & 4 & - & - \\
\hline CLAS & 0.90 & 12 & 0.91 & 11 & 1.0032 & 1.07 \\
\hline OLYMPUS & 1.57 & 20 & 0.64 & 19 & 1.0082 & 1.82 \\
\hline All & 1.46 & 36 & 0.96 & 34 & - & - \\
\hline \multicolumn{7}{|c|}{ Model: Borisyuk \& Kobushkin [58] } \\
\hline VEPP-3 & 2.28 & 4 & 2.28 & 4 & - & - \\
\hline CLAS & 1.02 & 12 & 0.94 & 11 & 1.0038 & 1.27 \\
\hline OLYMPUS & 2.15 & 20 & 0.75 & 19 & 1.0097 & 2.16 \\
\hline All & 1.79 & 36 & 1.00 & 34 & - & - \\
\hline \multicolumn{7}{|c|}{ Model: Bernauer et al. [35] } \\
\hline VEPP-3 & 1.90 & 4 & 1.90 & 4 & - & - \\
\hline CLAS & 0.74 & 12 & 0.90 & 11 & 0.9985 & -0.40 \\
\hline OLYMPUS & 0.46 & 20 & 0.51 & 19 & 1.0019 & 0.42 \\
\hline All & 0.71 & 36 & 0.80 & 34 & - & - \\
\hline
\end{tabular}

Table 3.4: Comparison of VEPP-3, CLAS, OLYMPUS, and the combined data set (All) to various TPE calculations showing the reduced $\chi^{2}$ value and the normalization factor $\mathcal{N}$ derived from the fit. The "No normalization" column represents a comparison when the normalization uncertainties of CLAS and OLYMPUS are added in quadrature. The column labelled "With normalization" is when the CLAS and OLYMPUS normalizations are allowed to float, as described in the text.

The results of these experiments are by no means definitive. The majority of the data are well below where the form factor discrepancy is significant $\left(Q^{2}>2 \mathrm{GeV}^{2}\right)$, so questions regarding the source of this discrepancy remain largely unanswered. There is a clear need for similar experiments at larger $Q^{2}$, and perhaps more importantly, at $\varepsilon<0.5$. Figure 2.10 shows that $R_{2 \gamma}$ remains small, even at $Q^{2}=2.5 \mathrm{GeV}^{2}$, for $\varepsilon>0.5$. This, of course, poses significant experimental difficulties due to the rapid drop in the elastic cross section at large lepton scattering angles. At the present time no new experiments have been approved for studies in the high- $Q^{2}$ region, so the question may remain unanswered for several years. There have been discussions in the community to produce an $e^{+}$beam at Jefferson Lab [135], but any such facility is uncertain and many years in the future.

An upcoming MUSE experiment [136] at Paul Scherrer Institute (PSI) will address the problem of the proton radius $[3,137]$ via precision measurements at small transferred momenta. MUSE will provide a comparison of electron and positron scattering on the proton, as well as positive and negative muons, directly constraining TPE for these processes at very low $Q^{2}$.

Effects due to TPE have been sought in experiments on polarization observables. Polarization measurements [68], where the real part of TPE could alter the angular dependence of the recoil proton polarization, have not observed substantial deviations of the polarization ratio, but reported deviations in the individual polarization components. Single-spin asymmetries (SSA) caused by normal (with respect to scattering plane) polarization provide a probe of the imaginary part of TPE amplitude. The data on beam SSA [86, 88] are in good agreement with unitarity-based calculations [82-85] for the 

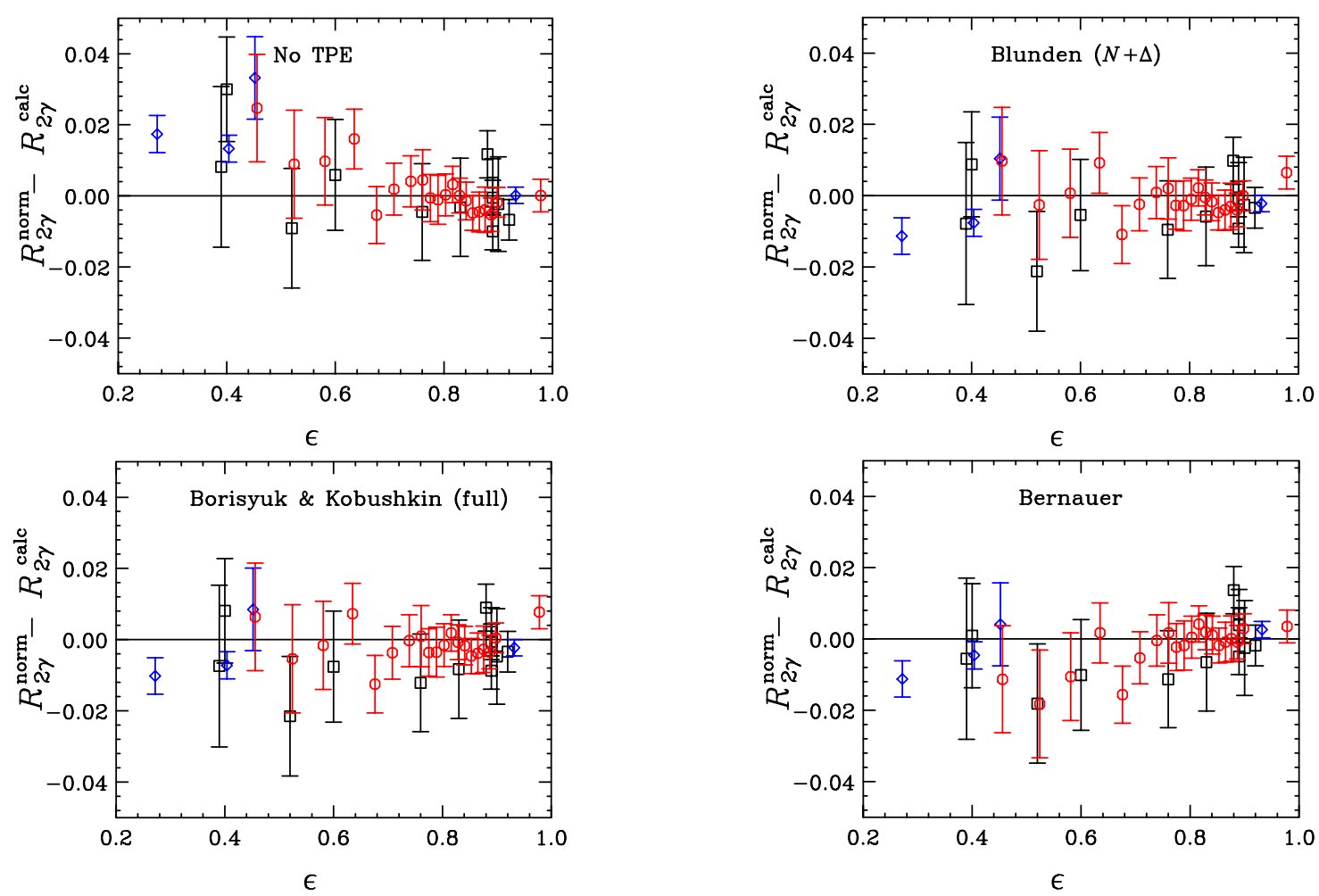

Figure 3.17: Difference between normalized $R_{2 \gamma}$ and model predictions as a function of $\varepsilon$. Data symbols are the same as in Fig. 3.15.

proton and light nuclei, but disagree with the data on a high- $Z$ target ${ }^{208} \mathrm{~Pb}$ both in sign and magnitude, possibly due to Coulomb distortion effects. The measurements of single-spin target asymmetry [77] in quasi-elastic scattering on a transversely polarized ${ }^{3} \mathrm{He}$ target showed a TPE effect that agreed with GPD predictions at high momentum transfer. The data both on target and beam SSA show evidence of inelastic excitations of the intermediate hadronic state and provide valuable input for theoretical constraints of TPE.

On the theoretical front, there has been significant progress in calculations of TPE based on the use of dispersion relations $[54,58,59]$. The use of spin- $-1 / 2$ and spin- $3 / 2$ helicity amplitudes from electroproduction data throughout the resonance region is a notable advance [58]. At forward angles and low $Q^{2}$ the dispersive approach allows one to use total photonucleon cross section data to constrain hadronic uncertainties [60,62]. Connecting the low to moderate $Q^{2}$ hadronic models with the high $Q^{2}$ QCD-based models studied in Refs. [48-50,65] remains an elusive goal.

Another area where progress might be made is regarding higher order radiative corrections. The large difference between exponentiated and non-exponentiated radiative corrections that increase with decreasing $\varepsilon$ suggests higher order corrections may be warranted. In addition, a reanalysis of the existing form factor and polarization data to uniformly apply and update the radiative corrections might provide further insight into the TPE process and the role it has in lepton-nucleon scattering.

\section{Acknowledgements}

This material is based upon work supported by NSERC (Canada) and the U.S. Department of Energy, Office of Science, Office of Nuclear Physics under contracts DE-AC05-06OR23177, DE-SC0013620, and DE-FG02-94ER40818. AA acknowledges support of the Gus Weiss endowment at George Washington University. PGB thanks Jefferson Lab for support during a sabbatical leave, where part of this work was completed. 


\section{References}

\section{References}

[1] C. E. Carlson and M. Vanderhaeghen, Ann. Rev. Nucl. Part. Sci. 57, 171 (2007), arXiv:hep-ph/0701272.

[2] J. Arrington, P. G. Blunden, and W. Melnitchouk, Prog. Part. Nucl. Phys. 66, 782 (2011), arXiv:1105.0951.

[3] R. Pohl, R. Gilman, G. A. Miller, and K. Pachucki, Ann. Rev. Nucl. Part. Sci. 63, 175 (2013), arXiv:1301.0905.

[4] C. E. Carlson, Prog. Part. Nucl. Phys. 82, 59 (2015), arXiv:1502.05314.

[5] M. N. Rosenbluth, Phys. Rev. 79, 615 (1950).

[6] Y.-S. Tsai, Phys. Rev. 122, 1898 (1961).

[7] L. W. Mo and Y.-S. Tsai, Rev. Mod. Phys. 41, 205 (1969).

[8] L. C. Maximon and J. A. Tjon, Phys. Rev. C62, 054320 (2000), arXiv:nucl-th/0002058.

[9] R. C. Walker et al., Phys. Rev. D49, 5671 (1994).

[10] E94110, M. E. Christy et al., Phys. Rev. C70, 015206 (2004), arXiv:nucl-ex/0401030.

[11] I. A. Qattan et al., Phys. Rev. Lett. 94, 142301 (2005), arXiv:nucl-ex/0410010.

[12] A. I. Akhiezer and M. Rekalo, Sov. J. Part. Nucl. 4, 277 (1974), [Fiz. Elem. Chast. Atom. Yadra 4, $662(1973)]$.

[13] R. G. Arnold, C. E. Carlson, and F. Gross, Phys. Rev. C23, 363 (1981).

[14] J. Arrington, Phys. Rev. C68, 034325 (2003), arXiv:nucl-ex/0305009.

[15] V. Punjabi et al., Phys. Rev. C71, 055202 (2005), arXiv:nucl-ex/0501018, [Erratum: Phys. Rev. C71, 069902 (2005)].

[16] A. J. R. Puckett et al., Phys. Rev. Lett. 104, 242301 (2010), arXiv:1005.3419.

[17] A. J. R. Puckett et al., Phys. Rev. C85, 045203 (2012), arXiv:1102.5737.

[18] X. Zhan et al., Phys. Lett. B705, 59 (2011), arXiv:1102.0318.

[19] Jefferson Lab Hall A, G. Ron et al., Phys. Rev. C84, 055204 (2011), arXiv:1103.5784.

[20] CLAS, D. Rimal et al., (2016), arXiv:1603.00315.

[21] Jefferson Lab Hall A, M. K. Jones et al., Phys. Rev. Lett. 84, 1398 (2000), arXiv:nucl-ex/9910005.

[22] O. Gayou et al., Phys. Rev. C64, 038202 (2001).

[23] Jefferson Lab Hall A, O. Gayou et al., Phys. Rev. Lett. 88, 092301 (2002), arXiv:nucl-ex/0111010.

[24] G. MacLachlan et al., Nucl. Phys. A764, 261 (2006). 
[25] G. Ron et al., Phys. Rev. Lett. 99, 202002 (2007), arXiv:0706.0128.

[26] C. B. Crawford et al., Phys. Rev. Lett. 98, 052301 (2007), arXiv:nucl-ex/0609007.

[27] Resonance Spin Structure, M. K. Jones et al., Phys. Rev. C74, 035201 (2006), arXiv:nucl-ex/0606015.

[28] P. G. Blunden, W. Melnitchouk, and J. A. Tjon, Phys. Rev. Lett. 91, 142304 (2003), arXiv:nucl-th/0306076.

[29] P. A. M. Guichon and M. Vanderhaeghen, Phys. Rev. Lett. 91, 142303 (2003), arXiv:hep-ph/0306007.

[30] S. Kondratyuk, P. G. Blunden, W. Melnitchouk, and J. A. Tjon, Phys. Rev. Lett. 95, 172503 (2005), arXiv:nucl-th/0506026.

[31] P. G. Blunden, W. Melnitchouk, and J. A. Tjon, Phys. Rev. C72, 034612 (2005), arXiv:nucl-th/0506039.

[32] G. 't Hooft and M. J. G. Veltman, Nucl. Phys. B153, 365 (1979).

[33] G. Passarino and M. J. G. Veltman, Nucl. Phys. B160, 151 (1979).

[34] T. Hahn and M. Perez-Victoria, Comput. Phys. Commun. 118, 153 (1999), arXiv:hep-ph/9807565.

[35] A1, J. C. Bernauer et al., Phys. Rev. C90, 015206 (2014), arXiv:1307.6227.

[36] S. Kondratyuk and P. G. Blunden, Phys. Rev. C75, 038201 (2007), arXiv:nucl-th/0701003.

[37] J. A. Tjon and W. Melnitchouk, Phys. Rev. Lett. 100, 082003 (2008), arXiv:0711.0143.

[38] J. A. Tjon, P. G. Blunden, and W. Melnitchouk, Phys. Rev. C79, 055201 (2009), arXiv:0903.2759.

[39] K. Nagata, H. Q. Zhou, C. W. Kao, and S. N. Yang, Phys. Rev. C79, 062501 (2009), arXiv:0811.3539.

[40] H. Q. Zhou, C. W. Kao, S. N. Yang, and K. Nagata, Phys. Rev. C81, 035208 (2010), arXiv:0910.3307.

[41] J. Arrington, W. Melnitchouk, and J. A. Tjon, Phys. Rev. C76, 035205 (2007), arXiv:0707.1861.

[42] W. A. McKinley and H. Feshbach, Phys. Rev. 74, 1759 (1948).

[43] I. G. Aznauryan and V. D. Burkert, Prog. Part. Nucl. Phys. 67, 1 (2012), arXiv:1109.1720.

[44] H.-Q. Zhou and S. N. Yang, Eur. Phys. J. A51, 105 (2015), arXiv:1407.2711.

[45] K. M. Graczyk, Phys. Rev. C88, 065205 (2013), arXiv:1306.5991.

[46] I. T. Lorenz, U.-G. Meissner, H. W. Hammer, and Y. B. Dong, Phys. Rev. D91, 014023 (2015), arXiv:1411.1704.

[47] Y. C. Chen, A. Afanasev, S. J. Brodsky, C. E. Carlson, and M. Vanderhaeghen, Phys. Rev. Lett. 93, 122301 (2004), arXiv:hep-ph/0403058. 
[48] A. V. Afanasev, S. J. Brodsky, C. E. Carlson, Y.-C. Chen, and M. Vanderhaeghen, Phys. Rev. D72, 013008 (2005), arXiv:hep-ph/0502013.

[49] D. Borisyuk and A. Kobushkin, Phys. Rev. D79, 034001 (2009), arXiv:0811.0266.

[50] N. Kivel and M. Vanderhaeghen, Phys. Rev. Lett. 103, 092004 (2009), arXiv:0905.0282.

[51] M. Gorchtein, Phys. Lett. B644, 322 (2007), arXiv:hep-ph/0610378.

[52] D. Borisyuk and A. Kobushkin, Phys. Rev. C78, 025208 (2008), arXiv:0804.4128.

[53] O. Tomalak and M. Vanderhaeghen, Phys. Rev. D90, 013006 (2014), arXiv:1405.1600.

[54] P. G. Blunden and W. Melnitchouk, Jefferson Lab preprint JLAB-THY-17-2430, 2017.

[55] M. Froissart, Phys. Rev. 123, 1053 (1961).

[56] D. Borisyuk and A. Kobushkin, Phys. Rev. C86, 055204 (2012), arXiv:1206.0155.

[57] D. Borisyuk and A. Kobushkin, Phys. Rev. C89, 025204 (2014), arXiv:1306.4951.

[58] D. Borisyuk and A. Kobushkin, Phys. Rev. C92, 035204 (2015), arXiv:1506.02682.

[59] O. Tomalak and M. Vanderhaeghen, Eur. Phys. J. A51, 24 (2015), arXiv:1408.5330.

[60] M. Gorchtein, Phys. Rev. C90, 052201 (2014), arXiv:1406.1612.

[61] R. W. Brown, Phys. Rev. D1, 1432 (1970).

[62] O. Tomalak and M. Vanderhaeghen, Phys. Rev. D93, 013023 (2016), arXiv:1508.03759.

[63] A. V. Radyushkin, Phys. Rev. D58, 114008 (1998), arXiv:hep-ph/9803316.

[64] M. Guidal, M. V. Polyakov, A. V. Radyushkin, and M. Vanderhaeghen, Phys. Rev. D72, 054013 (2005), arXiv:hep-ph/0410251.

[65] N. Kivel and M. Vanderhaeghen, JHEP 04, 029 (2013), arXiv:1212.0683.

[66] J. Mar et al., Phys. Rev. Lett. 21, 482 (1968).

[67] J. Guttmann, N. Kivel, M. Meziane, and M. Vanderhaeghen, Eur. Phys. J. A47, 77 (2011), arXiv:1012.0564.

[68] GEp2gamma, M. Meziane et al., Phys. Rev. Lett. 106, 132501 (2011), arXiv:1012.0339.

[69] P. G. Blunden, W. Melnitchouk, and J. A. Tjon, Phys. Rev. C81, 018202 (2010), arXiv:0911.3619.

[70] A. O. Barut and C. Fronsdal, Phys. Rev. 120, 1871 (1960).

[71] L. J. Dixon and M. Schreiber, Phys. Rev. D69, 113001 (2004), arXiv:hep-ph/0402221, [Erratum: Phys. Rev. D71, 059903 (2005)].

[72] SLAC E158, P. L. Anthony et al., Phys. Rev. Lett. 95, 081601 (2005), arXiv:hep-ex/0504049.

[73] A. De Rujula, J. M. Kaplan, and E. De Rafael, Nucl. Phys. B35, 365 (1971).

[74] A. De Rujula, J. M. Kaplan, and E. De Rafael, Nucl. Phys. B53, 545 (1973).

[75] B. Pasquini and M. Vanderhaeghen, Phys. Rev. C70, 045206 (2004), arXiv:hep-ph/0405303. 
[76] F. E. Maas et al., Phys. Rev. Lett. 94, 082001 (2005), arXiv:nucl-ex/0410013.

[77] Y. W. Zhang et al., Phys. Rev. Lett. 115, 172502 (2015), arXiv:1502.02636.

[78] A. Afanasev, I. Akushevich, and N. P. Merenkov, pp. 142-150, 2002, arXiv:hep-ph/0208260.

[79] N. Mott, Proc. R. Soc. (London) A 124, 425 (1929).

[80] E. D. Cooper and C. J. Horowitz, Phys. Rev. C72, 034602 (2005), arXiv:nucl-th/0506034.

[81] D. H. Jakubassa-Amundsen, J. Phys. G41, 075103 (2014).

[82] A. V. Afanasev and N. P. Merenkov, Phys. Rev. D70, 073002 (2004), arXiv:hep-ph/0406127.

[83] A. V. Afanasev and N. P. Merenkov, Phys. Lett. B599, 48 (2004), arXiv:hep-ph/0407167.

[84] M. Gorchtein, Phys. Rev. C73, 035213 (2006), arXiv:hep-ph/0512106.

[85] M. Gorchtein and C. J. Horowitz, Phys. Rev. C77, 044606 (2008), arXiv:0801.4575.

[86] HAPPEX, PREX, S. Abrahamyan et al., Phys. Rev. Lett. 109, 192501 (2012), arXiv:1208.6164.

[87] C. J. Horowitz, K. S. Kumar, and R. Michaels, Eur. Phys. J. A50, 48 (2014), arXiv:1307.3572.

[88] QWeak, B. P. Waidyawansa, Beam Normal Single Spin Asymmetry Measurements from QWeak, 2016, arXiv:1604.04602.

[89] V. Tvaskis et al., Phys. Rev. C73, 025206 (2006), arXiv:nucl-ex/0511021.

[90] A. V. Afanasev and C. E. Carlson, Phys. Rev. Lett. 94, 212301 (2005), arXiv:hep-ph/0502128.

[91] E. Tomasi-Gustafsson and G. I. Gakh, Phys. Rev. C72, 015209 (2005), arXiv:hep-ph/0412137.

[92] M. P. Rekalo, E. Tomasi-Gustafsson, and D. Prout, Phys. Rev. C60, 042202 (1999).

[93] J. Arrington, Phys. Rev. C69, 032201 (2004), arXiv:nucl-ex/0311019.

[94] J. Arrington, AIP Conf. Proc. 1160, 13 (2009), arXiv:0905.0713.

[95] CLAS, M. Moteabbed et al., Phys. Rev. C88, 025210 (2013), arXiv:1306.2286.

[96] D. Yount and J. Pine, Phys. Rev. 128, 1842 (1962).

[97] A. Browman, F. Liu, and C. Schaerf, Phys. Rev. 139, B1079 (1965).

[98] R. L. Anderson et al., Phys. Rev. Lett. 17, 407 (1966).

[99] W. Bartel et al., Phys. Lett. B25, 242 (1967).

[100] G. L. Cassiday et al., Phys. Rev. Lett. 19, 1191 (1967).

[101] R. L. Anderson et al., Phys. Rev. 166, 1336 (1968).

[102] B. Bouquet et al., Phys. Lett. B26, 178 (1968).

[103] S. Hartwig et al., Lett. Nuovo Cim. 12, 30 (1975).

[104] J. Arrington et al., (2004), arXiv:0408020.

[105] I. A. Rachek et al., Phys. Rev. Lett. 114, 062005 (2015), arXiv:1411.7372. 
[106] CLAS, D. Adikaram et al., Phys. Rev. Lett. 114, 062003 (2015), arXiv:1411.6908.

[107] B. S. Henderson et al., Phys. Rev. Lett. 118, 092501 (2016), arXiv:1611.04685.

[108] SAMPLE, S. P. Wells et al., Phys. Rev. C63, 064001 (2001), arXiv:nucl-ex/0002010.

[109] SAMPLE, D. T. Spayde et al., Phys. Rev. Lett. 84, 1106 (2000), arXiv:nucl-ex/9909010.

[110] P. LaViolette et al., E158 Report No. 70, 2009 (unpublished).

[111] G0, D. S. Armstrong et al., Phys. Rev. Lett. 95, 092001 (2005), arXiv:nucl-ex/0506021.

[112] G0, D. Androic et al., Nucl. Instrum. Meth. A646, 59 (2011), arXiv:1103.0761.

[113] G0, D. S. Armstrong et al., Phys. Rev. Lett. 99, 092301 (2007), arXiv:0705.1525.

[114] G0, D. Androic et al., Phys. Rev. Lett. 107, 022501 (2011), arXiv:1103.3667.

[115] Qweak, T. Allison et al., Nucl. Instrum. Meth. A781, 105 (2015), arXiv:1409.7100.

[116] A. V. Gramolin et al., Nucl. Phys. Proc. Suppl. 225-227, 216 (2012), arXiv:1112.5369.

[117] J. Arrington, Eur. Phys. J. A17, 311 (2003), arXiv:hep-ph/0209243.

[118] A. V. Gramolin et al., J. Phys. G41, 115001 (2014), arXiv:1401.2959.

[119] E. Tomasi-Gustafsson, M. Osipenko, E. A. Kuraev, and Yu. Bystritsky, Phys. Atom. Nucl. 76, 937 (2013), arXiv:0909.4736.

[120] J. Arrington and I. Sick, Phys. Rev. C70, 028203 (2004), arXiv:nucl-ex/0406014.

[121] I. A. Qattan, A. Alsaad, and J. Arrington, Phys. Rev. C84, 054317 (2011), arXiv:1109.1441.

[122] CLAS, B. A. Mecking et al., Nucl. Instrum. Meth. A503, 513 (2003).

[123] R. Ent et al., Phys. Rev. C64, 054610 (2001).

[124] L. Andivahis et al., Phys. Rev. D50, 5491 (1994).

[125] R. Milner et al., Nucl. Instrum. Meth. A741, 1 (2014).

[126] J. C. Bernauer et al., Nucl. Instrum. Meth. A755, 20 (2014), arXiv:1404.0579.

[127] D. Hasell et al., Nucl. Instrum. Meth. A603, 247 (2009).

[128] R. Pérez Benito et al., Nucl. Instrum. Meth. A826, 6 (2016), arXiv:1602.01702.

[129] J. C. Bernauer et al., Nucl. Instrum. Meth. A823, 9 (2016).

[130] A. Schmidt, PhD thesis, Massachusetts Institute of Technology, Cambridge, Massachusetts, 2016.

[131] B. S. Henderson, PhD thesis, Massachusetts Institute of Technology, Cambridge, Massachusetts, 2016.

[132] R. L. Russell, PhD thesis, Massachusetts Institute of Technology, Cambridge, Massachusetts, 2016.

[133] C. O'Connor, PhD thesis, Massachusetts Institute of Technology, Cambridge, Massachusetts, 2017. 
[134] L. D. Ice, PhD thesis, Arizona State University, Tempe, Arizona, 2016.

[135] E. Voutier, Nucl. Theor. 33, 142 (2014), arXiv:1412.1249.

[136] MUSE, R. Gilman et al., (2013), arXiv:1303.2160.

[137] R. Pohl et al., Nature 466, 213 (2010). 